\title{
Constant mean curvature surfaces of Delaunay type along a closed geodesic
}

\author{
Shiguang $\mathrm{Ma}^{*}$
}

November 1, 2021

\begin{abstract}
In this paper, we construct Delaunay type constant mean curvature surfaces along a nondegenerate closed geodesic in a 3-dimensional Riemannian manifold.
\end{abstract}

\section{Contents}

1 Introduction 2

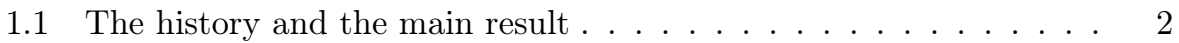

2 Geometry of Delaunay surfaces 4

2.1 The initial surface and the perturbation . . . . . . . . 4

2.1.1 Delaunay surfaces in Euclidean space $\mathbb{R}^{3} \ldots \ldots \ldots$. . . . .

2.1.2 Fermi coordinates and Taylor expansion of the metric near

the geodesic . . . . . . . . . . . . . 5

2.1.3 Initial Delaunay surfaces and the perturbation $\ldots \ldots \ldots$

2.2 The mean curvature of $\mathcal{D}_{\phi_{\tau_{0}}, p_{0}, \varepsilon}(w, \eta) \ldots \ldots \ldots \ldots$

2.2.1 The first fundamental form of $\mathcal{D}_{\phi_{\tau_{0}}, p_{0}, \varepsilon}(w, \eta) \ldots \ldots$

2.2 .2 Normal vector . . . . . . . . . . . . . . . . 10

2.2.3 The second fundamental form and the mean curvature of

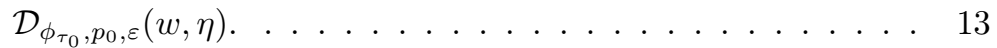

3 Jacobi operator 14

3.1 Basic properties . . . . . . . . . . . . . . . . . 14

3.2 High mode . . . . . . . . . . . . . . . . . . . . . . 15

3.3 1st mode . . . . . . . . . . . . . . . . . . 15

3.3 .1 Some preparations . . . . . . . . . . . . 16

3.3 .2 The main theorem of 1 st mode . . . . . . . . . 20

3.4 The sketch of the proof and main difficulties . . . . . . . . . . 21

3.5 0th mode . . . . . . . . . . . . . . . . 24 24

3.5.1 The main theorem of 0 th mode . . . . . . . . . . . . . 24

* School of Mathematical Sciences and LPMC, Nankai University, Tianjin, P.R.China, 300071, msgdyx8741@nankai.edu.cn. 
4 The existence of CMC surfaces 39

4.1 A fixed point argument . . . . . . . . . . . . . . . 39

4.2 The energy of the surface and the last step of the proof. . . . . 44

A The calculation of the mean curvature 47

B The proof of Lemma 3.1 and Lemma 3.6

C The proof of Lemma 3.11

D The proof of Lemma 3.15

E The proof of Lemma 3.17

\section{Introduction}

\subsection{The history and the main result}

Constant mean curvature (CMC) surfaces are a class of important submanifolds. Let $\left(M^{m+1}, g\right)$ be a Riemannian manifold of $m+1$ dimension. We consider the embedded CMC hypersurfaces. In early 1990s, R. Ye proved in 17 the existence of the foliation of constant mean curvature spheres in Riemannian manifolds around the nondegenerate critical points of the scalar curvature. In 1996, in [5] G. Huisken and S.T. Yau proved the existence of constant mean curvature foliation in the asymptotically flat end (of a manifold) with positive mass. Huisken and Yau's result were extended by L. Huang in [4] and C. Nerz in [11. Similar problems were also considered in asymptotically hyperbolic manifolds. For this topic, see [15, 12, 13, 10]. In [14], Pacard and Xu proved the existence of constant mean curvature spheres around the degenerate critical points of scalar curvature which can be regarded as a complement of Ye's result. In [9], Mazzeo and Pacard proved the existence of constant mean curvature tubes along a closed nondegenerate geodesic. Geodesic is a kind of simple minimal submanifold. In [7, Mahmoudi, Mazzeo and Pacard proved the existence of constant mean curvature hypersurfaces along minimal submanifolds. In contrast to the result of R. Ye in [17, the CMC hypersurfaces constructed in [9] and [7] constitute a partial foliation, that is a foliation with gaps. There is a good reason for such gaps, around which, bifurcation occurs. In particular, the CMC surfaces which bifurcate from the tubes are of Delaunay type, which are the main objects those will be constructed in this paper.

We will give the definition of Delaunay surfaces in the next section. The Delaunay surfaces were discovered in 1841 by C. Delaunay in [2. It is a one parameter family of complete non-compact surfaces in $\mathbb{R}^{3}$. The Delaunay surfaces have rotational symmetry, so they are generated by an ODE. One may refer to 3 for a description of Delaunay surfaces. They play an analogous role in the theory of complete CMC surfaces as catenoids do in the theory of complete minimal surfaces. In [16], it is proved that any complete minimal immersion 
$M^{n} \subset \mathbb{R}^{n+1}$ with two embedded ends and with finite total curvature must be a catenoid or a pair of planes. And it is proved in [6] that any CMC surface embedded in $\mathbb{R}^{3}$ having two ends is a Delaunay surface. Another fact is that any end of a complete minimal surface in $\mathbb{R}^{3}$ of finite total curvature must be asymptotic to a catenoid or a plane. Parallelling to this fact, each end of an embedded CMC surface with finite topology converges exponentially to the end of some Delaunay surface. Delaunay surfaces can be generalized in a proper way as CMC hypersurfaces which exists in $\mathbb{S}^{n}, \mathbb{R}^{n}$ and $\mathbb{H}^{n}$. In the more recent work [1, Bettiol and Piccione managed to construct Delaunay type CMC surfaces in cohomogeneity one manifolds. Cohomogeneity one manifolds are those support an isometric action of a Lie group such that the orbit space $M / G$ is one dimensional. We see that the metrics of cohomogeneity one manifolds are not generic. For generic metrics, the existence of Delaunay type CMC surfaces is unknown, despite some partial results. In this paper we focus on the existence of Delaunay type CMC surfaces along closed geodesics in generic metrics. As mentioned just now, this kind of surfaces can be regarded as the bifurcation branches of the CMC tubes constructed in 9]. One can refer to [9] for a description of the moduli space of CMC surfaces along $\Gamma$ which are isotopic to geodesic tubes, which is the motivation of this paper.

Let's state the main theorem roughly:

Theorem 1.1. Suppose $\left(M^{3}, g\right)$ is a 3-dimensional Riemannian manifold and $\Gamma$ is a simple closed embedded geodesic with nondegenerate Jacobi operator. Then for any $\tau_{0} \in\left(0, \frac{1}{4}\right)$ we can find $\varepsilon_{0}>0$ which depends on the manifold $M$ and $\tau_{0}$ such that there is a monotone sequence $\varepsilon_{n} \rightarrow 0$ with $\varepsilon_{0}>\varepsilon_{1}>\cdots>\varepsilon_{n}>\cdots$ such that along the geodesic there are at least two embedded Delaunay type CMC surfaces of size $\varepsilon_{n}$, with mean curvature $2 / \varepsilon_{n}$ and with Delaunay parameter close to $\tau_{0}$.

The terms "Delaunay type", "size $\varepsilon_{n}$ " and "Delaunay parameter close to $\tau_{0}$ " will be made clear in Theorem 2.3 which is the rigorous version of this theorem. The assumption that the Jacobi operator is nondegenerate is a mild restriction which holds for generic metrics. Our method can also be used in the case that there is symmetry.

Corollary 1.2. If the metric has rotational symmetry (at least in a tubular neighborhood of $\Gamma$ ) with $\Gamma$ being the axis, then in Theorem 1.1 we can remove the condition that the geodesic is nondegenerate.

In [7, the authors also did similar things as in [9], namely, if $\Sigma \subset M$ is a compact nondegenerate closed minimal submanifold and $\Sigma$ is at least of codimension two, then the authors constructed a partial foliation of CMC hypersurfaces condensing along $\Sigma$. The authors believe that there should be also bifurcation phenomenon in this setting. However geometric picture of the bifurcating CMC surfaces is still not clear in this case.

We use the perturbation method to solve this problem. Yet the argument is long and involved. This paper is organized as follows: 
In Section 2, we revise some basic facts of Delaunay undulo ${ }^{-} \mathrm{d}$ (which we call Delaunay surface) embedded in the Euclidean space $\mathbb{R}^{3}$ and we make a description on how we arrange an initial surface along the closed geodesic and how we perturb the initial surface. Then after long calculations we get the expression 13 for the mean curvature of the perturbed initial surface.

In Section 3 we analyze the Jacobi operator of the perturbed initial surface. We divide the function space into 3 parts, according to the invariant subspaces of the Jacobi operator. When restricting the Jacobi operator to each part we have high mode, 1st mode and 0th mode.

For high mode, it is easy to prove that the operator is invertible and the inverse has good bounds. The main result of high mode is contained in Subsection 3.2 .

For 1st mode, after careful examination, we find the Jacobi operator converges in certain sense to the Jacobi operator of the geodesic, which is invertible by the assumption that the geodesic is nondegenerate. Here we prove an "average 1" lemma (Lemma 3.1), which verifies the convergence. The main result of 1st mode is contained in Theorem 3.5 .

The reader may refer to Subsection 3.4 for the sketch of the whole procedure and the main difficulties of the proof. Due to the difficulties, in 0th mode, we consider a nonlinear ODE and prove an "average 0" lemma. The reader is suggested to understand Subsection 3.4 before going deep into the details of 0th mode. In Subsection 3.5 we analyze the 0th mode. The main theorem of 0th mode is Theorem 3.7. whose proof takes 6 steps.

In Section 4, we will prove the existence of the Delaunay type CMC surfaces, using a fixed point argument. One can also refer to Subsection 3.4 for the sketch of this section.

\section{Geometry of Delaunay surfaces}

\subsection{The initial surface and the perturbation}

\subsubsection{Delaunay surfaces in Euclidean space $\mathbb{R}^{3}$}

First we give a brief revision of the definition of Delaunay surfaces in Euclidean space $\mathbb{R}^{3}$. There are two kinds of Delaunay surfaces in $\mathbb{R}^{3}$, Delaunay nodo $\tilde{A}^{-}$ds and Delaunay undulo $\tilde{A}^{-}$ds. The first type can be immersed into $\mathbb{R}^{3}$, and the second type can be embedded into $\mathbb{R}^{3}$. In this paper, by Delaunay surface, we always mean Delaunay undulo $\tilde{\mathrm{A}}^{-} \mathrm{d}$. The Delaunay undulo $\widetilde{\mathrm{A}}^{-} \mathrm{d} D_{\tau_{0}}, 0<\tau_{0}<\frac{1}{4}$ can be parameterized by

$$
X_{\tau_{0}}(s, \theta):=\left(\phi_{\tau_{0}}(s) \cos \theta, \phi_{\tau_{0}}(s) \sin \theta, \psi_{\tau_{0}}(s)\right),
$$

where $(s, \theta) \in \mathbb{R} \times S^{1}$ and $(\phi, \psi)$ is the solution to the following system

$$
\begin{cases}\dot{\phi}^{2}+\left(\phi^{2}+\tau_{0}\right)^{2}=\phi^{2}, & \phi(0)=\frac{1-\sqrt{1-4 \tau_{0}}}{2}, \\ \dot{\psi}=\phi^{2}+\tau_{0}, & \psi(0)=0,\end{cases}
$$

where the derivative "." is taken with respect to parameter $s$. 
From $\dot{\psi}=\phi^{2}+\tau_{0}, \psi$ is strictly increasing in $s$. So we can also regard $\phi$ as a function of $\psi$. Easy calculation yields, $\phi(\psi)$ satisfies

$$
\left\{\begin{array}{l}
\phi_{\psi \psi}-\phi^{-1}\left(1+\phi_{\psi}^{2}\right)+2\left(1+\phi_{\psi}^{2}\right)^{\frac{3}{2}}=0 \\
\phi(0)=\frac{1-\sqrt{1-4 \tau_{0}}}{2} \\
\phi_{\psi}(0)=0
\end{array}\right.
$$

which can be regarded as an equivalent definition of Delaunay surfaces. One may refer to [8] for more information of Delaunay surfaces. Direct calculations shows that $X_{\tau_{0}}(s, \theta)=(\phi \cos \theta, \phi \sin \theta, \psi) \subset\left(\mathbb{R}^{3}, g_{\text {edu }}\right)$ has mean curvature

$$
-\phi_{\psi \psi}\left(1+\phi_{\psi}^{2}\right)^{-3 / 2}+\phi^{-1}\left(1+\phi_{\psi}^{2}\right)^{-1 / 2} \equiv 2,
$$

which is independent of $\tau_{0} \in\left(0, \frac{1}{4}\right)$.

Remark. Easy calculation gives that $\dot{\phi}, \dot{\psi}, \ddot{\phi}, \ddot{\psi}, \tau_{0}$ can be expressed as functions of $\left(\phi, \phi_{\psi}\right)$.

Remark. The solution to (1) or (2) is periodic because

$$
\tau\left(\phi, \phi_{\psi}\right)=-\phi^{2}+\frac{\phi}{\sqrt{1+\phi_{\psi}^{2}}} \equiv \tau_{0} .
$$

And the solution satisfies

$$
\frac{1-\sqrt{1-4 \tau_{0}}}{2} \leq \phi(\psi) \leq \frac{1+\sqrt{1-4 \tau_{0}}}{2} .
$$

So $\phi(\psi)$ attains its minimum at $\psi=0$.

\subsubsection{Fermi coordinates and Taylor expansion of the metric near the geodesic}

From now on we discuss the geometry of Delaunay type surface along a geodesic in a Riemannian 3-manifold. Fix an arc length parametrization $x_{0}$ of the geodesic $\Gamma, x_{0} \in\left[0, L_{\Gamma}\right]$, where $L_{\Gamma}$ is the length of $\Gamma$. We denote the normal bundle of $\Gamma$ by $N \Gamma$. Choose a parallel orthonormal basis $E_{1}, E_{2}$ for $N \Gamma$ (say along $[a, b]$ ) which determines a coordinate system

$$
x:\left(x_{0}, x_{1}, x_{2}\right) \mapsto \exp _{\Gamma\left(x_{0}\right)}\left(x_{1} E_{1}+x_{2} E_{2}\right):=F(x),
$$

and we denote the corresponding coordinate vector fields by $X_{\alpha}:=F_{*}\left(\partial_{x_{\alpha}}\right)$. We adopt the convention that indices $i, j, k, \cdots \in\{1,2\}$ while $\alpha, \beta, \cdots \in\{0,1,2\}$. Let $r=\sqrt{x_{1}^{2}+x_{2}^{2}}$. By Gauss' Lemma $r$ is the geodesic distance from $x$ to $\Gamma$ and the vector $\partial_{r}=\frac{1}{r}\left(x_{1} X_{1}+x_{2} X_{2}\right)$ is perpendicular to $X_{0}$. We also denote $\partial_{\theta}=-x_{2} X_{1}+x_{1} X_{2}$, where $(r, \theta)$ is the polar coordinate.

It is easy to see that the metric coefficients $g_{\alpha \beta}=<X_{\alpha}, X_{\beta}>$ equal $\delta_{\alpha \beta}$ along $\Gamma$. Now we are going to calculate higher order terms in the Taylor expansions 
of $g_{\alpha \beta}$. By the notation $\tilde{O}\left(r^{m}\right)$, we mean a function $f$ such that it and its partial derivatives of any order, with respect to the vector fields $X_{0}$ and $x_{i} X_{j}$, are bounded by $\mathrm{Cr}^{m}$ in some fixed tube $\left\{p \mid r(p, \Gamma) \leq r_{0}\right\}$.

First for the covariant derivative, we have

Lemma 2.1. For $\alpha, \beta=0,1,2$,

$$
\nabla_{X_{\alpha}} X_{\beta}=\sum_{\gamma=0}^{2} \tilde{O}(r) X_{\gamma}
$$

and more precisely for $\alpha=\beta=0$, we have

$$
\nabla_{X_{0}} X_{0}=-\sum_{i, j=1}^{2} R\left(X_{j}, X_{0}, X_{i}, X_{0}\right)_{p} x_{i} X_{j}+\sum_{\gamma=0}^{2} \tilde{O}\left(r^{2}\right) X_{\gamma}
$$

where $R\left(X_{i}, X_{j}, X_{k}, X_{l}\right)=<\nabla_{X_{i}} \nabla_{X_{j}} X_{k}-\nabla_{X_{j}} \nabla_{X_{i}} X_{k}-\nabla_{\left[X_{i}, X_{j}\right]} X_{k}, X_{l}>$.

Proof. We follow Lemma 2.1 in [9]. At any point $p \in \Gamma$

$$
\nabla_{X_{0}} X_{0}=\nabla_{X_{0}} X_{j}=\nabla_{X_{j}} X_{0}=\nabla_{X_{i}} X_{j}=0
$$

where the last one holds because on $\Gamma, \nabla_{X_{i}} X_{i}=0, \nabla_{X_{i}+X_{j}}\left(X_{i}+X_{j}\right)=0$. So the first equality follows. For the second one note that

$$
\begin{aligned}
X_{i}<\nabla_{X_{0}} X_{0}, X_{j}>_{p} & =<\nabla_{X_{i}} \nabla_{X_{0}} X_{0}, X_{j}>_{p}+<\nabla_{X_{0}} X_{0}, \nabla_{X_{i}} X_{j}>_{p} \\
& =<\nabla_{X_{i}} \nabla_{X_{0}} X_{0}, X_{j}>_{p}+\tilde{O}\left(r^{2}\right) \\
& =<R\left(X_{i}, X_{0}\right) X_{0}, X_{j}>_{p}+<\nabla_{X_{0}} \nabla_{X_{i}} X_{0}, X_{j}>_{p}+O\left(r^{2}\right) \\
& =<R\left(X_{i}, X_{0}\right) X_{0}, X_{j}>_{p}+\tilde{O}(r),
\end{aligned}
$$

which implies the second one.

The next lemma gives the expansion of the metric coefficients in Fermi coordinates.

Lemma 2.2. In the same notation as before, we have

$$
\left\{\begin{array}{l}
g_{i j}(q)=\delta_{i j}+\frac{1}{3} R\left(X_{k}, X_{i}, X_{l}, X_{j}\right)_{p} x_{k} x_{l}+\tilde{O}\left(r^{3}\right) \\
g_{0 i}(q)=\frac{2}{3} R\left(X_{k}, X_{0}, X_{l}, X_{i}\right)_{p} x_{k} x_{l}+\tilde{O}\left(r^{3}\right) \\
g_{00}(q)=1+R\left(X_{k}, X_{0}, X_{l}, X_{0}\right)_{p} x_{k} x_{l}+\tilde{O}\left(r^{3}\right)
\end{array}\right.
$$

Proof. The reader may refer to the proof of Proposition 2.1 in [9] for the proof . Here we give more accurate expansion for $g_{0 i}$. 


\subsubsection{Initial Delaunay surfaces and the perturbation}

First we arrange an initial Delaunay surface of size $\varepsilon$ along the geodesic. Fix a point $p_{0} \in \Gamma$ with $x_{0}\left(p_{0}\right)=0, \bmod L_{\Gamma}$ and a parameter $\tau_{0} \in(0,1 / 4)$. We assume $\phi_{\tau_{0}}(\psi)$ is the solution to (2). Suppose the smallest positive period of $\phi(\psi)$ is $\psi_{1}\left(\tau_{0}\right)$.

Definition 1. We define the set of "proper size" for $L_{\Gamma}$ and $\tau_{0}$ by

$$
\operatorname{PS}\left(L_{\Gamma}, \tau_{0}\right)=\left\{\varepsilon_{N}=\frac{L_{\Gamma}}{\psi_{1}\left(\tau_{0}\right) N} ; N \in \mathbb{N}^{+}\right\} .
$$

In the following, we always assume $\varepsilon \in \operatorname{PS}\left(L_{\Gamma}, \tau_{0}\right)$. We can arrange a Delaunay type initial surface of size $\varepsilon$ around the geodesic. Let's make it precise.

The unit circle bundle is locally trivialized by the map

$$
[a, b] \times S^{1} \ni\left(x_{0}, \Upsilon\right) \mapsto\left(\Gamma\left(x_{0}\right), \sum_{j=1}^{2} \Upsilon_{j} E_{j}\right) \in S N \Gamma .
$$

The image

$$
F\left(x_{0}, \varepsilon \phi_{\tau_{0}}\left(\frac{x_{0}}{\varepsilon}\right) \Upsilon\right)
$$

can be defined locally and extended globally, as it does not depend on the choice of orthonormal basis $E_{i}$. We denote the image by $\mathcal{D}_{\phi_{\tau_{0}}, p_{0}, \varepsilon}$.

Consider the following perturbation of $\mathcal{D}_{\phi_{\tau_{0}}, p_{0}, \varepsilon}$, denoted by $\mathcal{D}_{\phi_{\tau_{0}}, p_{0}, \varepsilon}(w, \eta)$, where $w$ is a function on unit circle bundle $S N \Gamma$ and $\eta$ is a section of $N \Gamma$.

Fix $\varepsilon>0$, and denote the image

$$
F\left(x_{0}, \varepsilon\left(\phi\left(\frac{x_{0}}{\varepsilon}\right)+w\left(\frac{x_{0}}{\varepsilon}, \theta\right)\right) \Upsilon+\eta\left(x_{0}\right)\right) ;
$$

by $\mathcal{D}_{\phi_{\tau_{0}}, p_{0}, \varepsilon}(w, \eta)$. It is obtained by first taking the vertical graph of the function $\varepsilon w$ over the initial Delaunay surface $\mathcal{D}_{\phi_{\tau_{0}}, p_{0}, \varepsilon}$ and then translating it by $\eta$.

First it is clear what do the derivatives with respect to $x_{0}, \theta$ mean for a function on $S N \Gamma$. For a smooth section $\eta$ of $N \Gamma$, locally we may write it as $\eta_{1} E_{1}+\eta_{2} E_{2}$. By $\frac{\partial \eta}{\partial x_{0}}$ we mean $\frac{\partial \eta_{1}}{\partial x_{0}} E_{1}+\frac{\partial \eta_{2}}{\partial x_{0}} E_{2}$ and it is similar for higher order derivatives. We will work in the following spaces.

- $C_{x_{0}}^{m}(\Gamma, N \Gamma), C_{x_{0}}^{m}(S N \Gamma), 0 \leq m \leq \infty$ are the spaces in which all functions have continuous derivatives up to order $m$, with respect to $x_{0}$ (and also $\theta$ for the second one). $C_{x_{0}}^{m, \alpha}(\Gamma, N \Gamma), C_{x_{0}}^{m, \alpha}(S N \Gamma), 0 \leq m<\infty, 0<\alpha<1$ are the usual HÃ $\uparrow l d e r$ spaces, where the derivatives are taken with respect to $x_{0}$ ( and also $\theta$ for the second one). If we replace $\alpha$ with 1 , they are the usual Lipschitz spaces. If we replace $x_{0}$ with $y_{0}$, then the derivatives are taken with respect to $y_{0}$ (and also $\theta$ if needed).

- $C_{\varepsilon}^{m, \alpha}(\Gamma, N \Gamma), C_{\varepsilon}^{m, \alpha}(S N \Gamma)$ are the modified HÃ $\uparrow l d e r$ spaces, where the derivatives are taken with respect to $\varepsilon x_{0}=\psi$ (and also $\theta$ for the second one). 
- $C_{x_{0}, \varepsilon}^{m, \alpha}(\Gamma, N \Gamma), C_{x_{0}, \varepsilon}^{m, \alpha}(S N \Gamma)$ are the modified HÃ $q$ lder spaces with $\|f\|_{C_{x_{0}, \varepsilon}^{m, \alpha}}=$ $\|f\|_{C^{0}}+\left\|\partial_{x_{0}} f\right\|_{C_{\varepsilon}^{m-1, \alpha}}$.

- $W_{\varepsilon}^{1,2}(S N \Gamma)$ is the modified Sobolev space, where the derivatives are taken with respect to $\psi$ and $\theta . W_{\varepsilon}^{-1,2}$ is the dual space of $W_{\varepsilon}^{1,2}$.

- $\|(f, g)\|_{\varepsilon, \alpha}=\|f\|_{C_{x_{0}}^{1}}+\varepsilon\|g\|_{C_{\varepsilon}^{\alpha}}$.

Sometimes we omit the symbols like $\Gamma, S N \Gamma$ in the norms when it is clear from the context.

For $p \in \Gamma$, let $S_{p}^{1}$ denote the unit circle fibre of $S N \Gamma$ over $p$. Any function $w$ on $S N \Gamma$ decomposes into a sum of three terms

$$
w=w_{0}+w_{1}+\tilde{w} .
$$

Here the restriction of any one of $w_{0}, w_{1}, \tilde{w}$ to each $S_{p}^{1}$ lies in the span of the eigenfunctions $\xi_{j}$ on $S^{1}$ with $j=0, j=1,2$, and $j>2$, respectively. $w_{0}$ is a function on $\Gamma$.

$$
\begin{aligned}
w_{1}(s, \theta) & =w_{1}^{1}(s) \xi_{1}+w_{1}^{2}(s) \xi_{2} \\
& =w_{1}^{1}(s) \cos \theta+w_{1}^{2}(s) \sin \theta .
\end{aligned}
$$

Note that any linear combination of $\xi_{1}$ and $\xi_{2}$ can be identified with a translation in $\mathbb{R}^{2}$ ( $\xi_{1}$ and $\xi_{2}$ correspond to the translations in $x$ and $y$ direction). Correspondingly, $w_{1}$ is canonically associated to a section $\eta$ of the normal bundle $N \Gamma$. At last

$$
\tilde{w}(s, \theta)=\sum_{j>2} \tilde{w}_{j}(s) \xi_{j} .
$$

We denote by $\Pi_{0}, \Pi_{1}$ and $\tilde{\Pi}$ the projections onto these three components respectively. We assume $\Pi_{1} w=0$ and the $\Pi_{1}$ part of $w$ is actually represented by $\eta$.

Now we can state Theorem 1.1 rigorously.

Theorem 2.3. Suppose that $\left(M^{3}, g\right)$ is a Riemannian manifold of 3 dimension and $\Gamma$ is a simple closed embedded geodesic with nondegenerate Jacobi operator. Then for any $\tau_{0} \in(0,1 / 4)$ there is $\varepsilon_{0}>0$ such that when $0<\varepsilon<\varepsilon_{0}$ and $\varepsilon \in \operatorname{PS}\left(L_{\Gamma}, \tau_{0}\right)$ and $i=1,2$, we have two different Delaunay type surfaces $\mathcal{D}_{\phi_{\tau_{0}}, p_{i}, \varepsilon}\left(w_{0, i}+\tilde{w}_{i}, \eta_{i}\right)$ along the geodesic which satisfy

$$
H\left(\mathcal{D}_{\phi_{\tau_{0}}, p_{i}, \varepsilon}\left(w_{0, i}+\tilde{w}_{i}, \eta_{i}\right)\right)=\frac{2}{\varepsilon} .
$$

Here $p_{i} \in \Gamma$ and $w_{0, i}, \eta_{i}, \tilde{w}_{i}$ belong to 0 th part, 1st part and high part respectively. Moreover for uniform constant $C$

$$
\varepsilon\left\|w_{0, i}\right\|_{C_{\varepsilon}^{2, \alpha}}+\left\|\eta_{i}\right\|_{C_{x_{0}, \varepsilon}^{2, \alpha}}+\left\|\tilde{w}_{i}\right\|_{C_{\varepsilon}^{2, \alpha}} \leq C \varepsilon^{2} .
$$




\subsection{The mean curvature of $\mathcal{D}_{\phi_{\tau_{0}}, p_{0}, \varepsilon}(w, \eta)$}

\subsubsection{The first fundamental form of $\mathcal{D}_{\phi_{\tau_{0}}, p_{0}, \varepsilon}(w, \eta)$}

Now we calculate the first fundamental form of $\mathcal{D}_{\phi_{\tau_{0}}, p_{0}, \varepsilon}(w, \eta)$ with respect to the coordinate $(s, \theta)$ at the point $q=F(\varepsilon \psi(s), \varepsilon(\phi(s)+w(s, \theta)) \Upsilon(\theta)+\eta(\varepsilon \psi(s)))$. Suppose $p=F(\varepsilon \psi(s), 0)$. First we have

$$
\left\{\begin{array}{l}
\partial_{s}=\varepsilon\left(\dot{\psi} X_{0}+\left(\dot{\phi}+\frac{\partial w}{\partial s}\right) \Upsilon+\dot{\psi} \frac{\partial \eta}{\partial x_{0}}\right) \\
\partial_{\theta}=\varepsilon\left((\phi+w) \Upsilon_{\theta}+\frac{\partial w}{\partial \theta} \Upsilon\right)
\end{array}\right.
$$

and $x_{k}(q)=\varepsilon(\phi(s)+w(s, \theta)) \Upsilon^{k}+\eta^{k}, k=1,2$.

Definition 2. In the following, $L(w, \eta)$ denotes any expression which is a linear differential operator (of order at most 2), which satisfies

$$
\|L(w, \eta)\|_{C_{\varepsilon}^{\alpha}} \leq C\left(\|w\|_{C_{\varepsilon}^{2, \alpha}(S N \Gamma)}+\|\eta\|_{C_{x_{0}, \varepsilon}^{2, \alpha}(\Gamma, N \Gamma)}\right),
$$

where $C$ is independent of $\rho$. Similarly, $Q(w, \eta)$ denotes any nonlinear differential operator (of order less than or equal to 2) in $w$ and $\eta$ which vanishes quadratically in the pair $(w, \eta)$ and such that

$$
\begin{aligned}
\left\|Q\left(w_{1}, \eta_{1}\right)-Q\left(w_{2}, \eta_{2}\right)\right\|_{C_{\varepsilon}^{\alpha}} \leq & C \sup _{i=1,2}\left(\left\|w_{i}\right\|_{C_{\varepsilon}^{2, \alpha}(S N \Gamma)}+\left\|\eta_{i}\right\|_{C_{x_{0}, \varepsilon}^{2, \alpha}(\Gamma, N \Gamma)}\right) \\
& \times\left(\left\|w_{1}-w_{2}\right\|_{C_{\varepsilon}^{2, \alpha}(S N \Gamma)}+\left\|\eta_{1}-\eta_{2}\right\|_{C_{x_{0}, \varepsilon}^{2, \alpha}(\Gamma, N \Gamma)}\right) .
\end{aligned}
$$

Here the spaces $C_{\varepsilon}^{\alpha}$ are either equal to $C_{\varepsilon}^{\alpha}(S N \Gamma)$ or $C_{\varepsilon}^{\alpha}(\Gamma, N \Gamma)$ according to the range of $L$ and $Q$. Finally, by $O\left(\varepsilon^{k}\right)$ we mean $\varepsilon^{k} E\left(\phi(\psi), \phi_{\psi}(\psi), \psi\right)$, where $E\left(\phi(\psi), \phi_{\psi}(\psi), \psi\right)$ denotes any smooth function with

$$
\left|\frac{\partial E\left(\phi, \phi_{\psi}, \psi\right)}{\partial \phi}\right|+\left|\frac{\partial E\left(\phi, \phi_{\psi}, \psi\right)}{\partial \phi_{\psi}}\right| \leq C\left(\tau_{0}\right),\left|\frac{\partial E\left(\phi, \phi_{\psi}, \psi\right)}{\partial \psi}\right| \leq C \varepsilon,
$$

where $C$ is a uniform constant.

From (3) we know

\section{Lemma 2.4.}

$$
\begin{aligned}
<X_{0}, X_{0}>_{q}= & 1+\varepsilon^{2} \phi^{2} R\left(\Upsilon, X_{0}, \Upsilon, X_{0}\right)_{p}+2 \varepsilon \phi R\left(\Upsilon, X_{0}, \eta, X_{0}\right)_{p} \\
& +\varepsilon^{2} L(w, \eta)+Q(w, \eta)+O\left(\varepsilon^{3}\right) \\
<X_{i}, X_{j}>_{q}= & \delta_{i j}+\frac{1}{3} \varepsilon^{2} \phi^{2} R\left(\Upsilon, X_{i}, \Upsilon, X_{j}\right)_{p}+\frac{1}{3} \varepsilon \phi\left(R\left(\Upsilon, X_{i}, \eta, X_{j}\right)_{p}\right. \\
& \left.+R\left(\eta, X_{i}, \Upsilon, X_{j}\right)_{p}\right)+\varepsilon^{2} L(w, \eta)+Q(w, \eta)+O\left(\varepsilon^{3}\right) \\
<X_{0}, X_{i}>_{q}= & \frac{2}{3} \varepsilon^{2} \phi^{2} R\left(\Upsilon, X_{0}, \Upsilon, X_{i}\right)_{p}+\frac{2}{3} \varepsilon \phi\left(R\left(\Upsilon, X_{0}, \eta, X_{i}\right)_{p}\right. \\
& \left.+R\left(\eta, X_{0}, \Upsilon, X_{i}\right)_{p}\right)+\varepsilon^{2} L(w, \eta)+Q(w, \eta)+O\left(\varepsilon^{3}\right) .
\end{aligned}
$$


We use these expansions to obtain the expansions of the first fundamental form,

\section{Lemma 2.5.}

$$
\begin{aligned}
\varepsilon^{-2}<\partial_{s}, \partial_{s}>= & \phi^{2}+\varepsilon^{2} \phi^{2} \dot{\psi}^{2} R\left(\Upsilon, X_{0}, \Upsilon, X_{0}\right)+2 \varepsilon \phi \dot{\psi}^{2} R\left(\Upsilon, X_{0}, \eta, X_{0}\right) \\
& +\frac{4}{3} \varepsilon \phi \dot{\phi} \dot{\psi} R\left(\Upsilon, X_{0}, \eta, \Upsilon\right)+2 \dot{\phi} \frac{\partial w}{\partial s}+2 \dot{\phi} \dot{\psi}<\Upsilon, \frac{\partial \eta}{\partial x_{0}}>_{e} \\
& +\varepsilon^{2} L(w, \eta)+Q(w, \eta)+O\left(\varepsilon^{3}\right), \\
\varepsilon^{-2}<\partial_{s}, \partial_{\theta}>= & \frac{2}{3} \varepsilon^{2} \phi^{3} \dot{\psi} R\left(\Upsilon, X_{0}, \Upsilon, \Upsilon_{\theta}\right)+\frac{2}{3} \varepsilon \phi^{2} \dot{\psi}\left(R\left(\Upsilon, X_{0}, \eta, \Upsilon_{\theta}\right)\right. \\
& \left.+R\left(\eta, X_{0}, \Upsilon, \Upsilon_{\theta}\right)\right)+\frac{1}{3} \varepsilon \phi^{2} \dot{\phi} R\left(\eta, \Upsilon, \Upsilon, \Upsilon_{\theta}\right)+\dot{\phi} \frac{\partial w}{\partial \theta} \\
& +\phi \dot{\psi}<\frac{\partial \eta}{\partial x_{0}}, \Upsilon_{\theta}>_{e}+\varepsilon^{2} L(w, \eta)+Q(w, \eta)+O\left(\varepsilon^{3}\right), \\
\varepsilon^{-2}<\partial_{\theta}, \partial_{\theta}>= & \phi^{2}+2 \phi w+\frac{1}{3} \varepsilon^{2} \phi^{4} R\left(\Upsilon, \Upsilon_{\theta}, \Upsilon, \Upsilon_{\theta}\right)+\frac{2}{3} \varepsilon \phi^{3} R\left(\Upsilon, \Upsilon_{\theta}, \eta, \Upsilon_{\theta}\right) \\
& +\varepsilon^{2} L(w, \eta)+Q(w, \eta)+O\left(\varepsilon^{3}\right) .
\end{aligned}
$$

Note that all the curvatures here and after are taken on $p \in \Gamma$.

Proof. The proof is direct calculation, using Lemma 2.4. For example for the first one $\varepsilon^{-2}<\partial_{s}, \partial_{s}>$, first we have

$$
\varepsilon^{-2}<\partial_{s}, \partial_{s}>=<\dot{\psi} X_{0}+\left(\dot{\phi}+\frac{\partial w}{\partial s}\right) \Upsilon+\dot{\psi} \frac{\partial \eta}{\partial x_{0}}, \dot{\psi} X_{0}+\left(\dot{\phi}+\frac{\partial w}{\partial s}\right) \Upsilon+\dot{\psi} \frac{\partial \eta}{\partial x_{0}}>
$$

And we get 6 different terms on the right hand side. For each one we can use Lemma 2.4. Finally we can proof the lemma.

\subsubsection{Normal vector}

Now we are going to find the expansion of the unit normal vector of $\mathcal{D}_{\phi_{\tau_{0}}, p_{0}, \varepsilon}(w, \eta)$. First we take

$$
N_{0}=\frac{1}{\phi}\left(\dot{\phi} X_{0}-\dot{\psi} \Upsilon\right)
$$

which is the unit normal vector of $\mathcal{D}_{\phi_{\tau_{0}}, p_{0}, \varepsilon}(0,0)$ when curvature vanishes. We may assume the unit normal vector $N$ of $\mathcal{D}_{\phi_{\tau_{0}}, p_{0}, \varepsilon}(w, \eta)$ has the form

$$
N=\frac{1}{k}\left(N_{0}+a_{1} \partial_{s}+a_{2} \partial_{\theta}\right)
$$

where $k$ is the norm of $N_{0}+a_{1} \partial_{s}+a_{2} \partial_{\theta}$. We will get the expansion for $a_{1}, a_{2}$ and $k$. First

$$
\left\{\begin{array}{l}
0=<k N, \partial_{s}>=<N_{0}, \partial_{s}>+a_{1}<\partial_{s}, \partial_{s}>+a_{2}<\partial_{s}, \partial_{\theta}> \\
0=<k N, \partial_{\theta}>=<N_{0}, \partial_{\theta}>+a_{1}<\partial_{s}, \partial_{\theta}>+a_{2}<\partial_{\theta}, \partial_{\theta}>
\end{array}\right.
$$




\section{Lemma 2.6.}

$$
\begin{aligned}
\varepsilon^{-1}<N_{0}, \partial_{s}>= & -\frac{\dot{\psi}}{\phi} \frac{\partial w}{\partial s}-\frac{\dot{\psi}^{2}}{\phi}<\frac{\partial \eta}{\partial x_{0}}, \Upsilon>_{e}+\varepsilon^{2} \phi \dot{\phi} \dot{\psi} R\left(\Upsilon, X_{0}, \Upsilon, X_{0}\right) \\
& +2 \varepsilon \dot{\phi} \dot{\psi} R\left(\Upsilon, X_{0}, \eta, X_{0}\right)+\frac{2}{3} \varepsilon\left(\dot{\phi}^{2}-\dot{\psi}^{2}\right) R\left(\Upsilon, X_{0}, \eta, \Upsilon\right) \\
& +\varepsilon^{2} L(w, \eta)+Q(w, \eta)+O\left(\varepsilon^{3}\right), \\
\varepsilon^{-1}<N_{0}, \partial_{\theta}>= & \frac{2}{3} \varepsilon^{2} \phi^{2} \dot{\phi} R\left(\Upsilon, X_{0}, \Upsilon, \Upsilon_{\theta}\right) \\
& +\frac{2}{3} \varepsilon \phi \dot{\phi}\left(R\left(\Upsilon, X_{0}, \eta, \Upsilon_{\theta}\right)+R\left(\eta, X_{0}, \Upsilon, \Upsilon_{\theta}\right)\right) \\
& +\varepsilon^{2} L(w, \eta)+Q(w, \eta)+O\left(\varepsilon^{3}\right) .
\end{aligned}
$$

Proof. The proof is again direct calculations using (5) and (6).

We denote $g_{s s}=<\partial_{s}, \partial_{s}>, g_{s \theta}=g_{\theta s}=<\partial_{s}, \partial_{\theta}>, g_{\theta \theta}=<\partial_{\theta}, \partial_{\theta}>$. From Lemma 2.5 we have

$$
\left(\begin{array}{ll}
g_{s s} & g_{s \theta} \\
g_{s \theta} & g_{\theta \theta}
\end{array}\right)=\varepsilon^{2} \phi^{2}\left(\begin{array}{cc}
1+\sigma_{1} & \sigma_{2} \\
\sigma_{2} & 1+\sigma_{3}
\end{array}\right)
$$

where

$$
\left\{\begin{aligned}
\sigma_{1} & =\varepsilon^{2} \dot{\psi}^{2} R\left(\Upsilon, X_{0}, \Upsilon, X_{0}\right)+2 \varepsilon \phi^{-1} \dot{\psi}^{2} R\left(\Upsilon, X_{0}, \eta, X_{0}\right) \\
& +\frac{4}{3} \varepsilon \phi^{-1} \dot{\phi} \dot{\psi} R\left(\Upsilon, X_{0}, \eta, \Upsilon\right)+2 \phi^{-2} \dot{\phi} \frac{\partial w}{\partial s} \\
& +2 \phi^{-2} \dot{\phi} \dot{\psi}<\Upsilon, \frac{\partial \eta}{\partial x_{0}}>_{e}+\varepsilon^{2} L(w, \eta)+Q(w, \eta)+O\left(\varepsilon^{3}\right), \\
\sigma_{2} & =\frac{2}{3} \varepsilon^{2} \phi \dot{\psi} R\left(\Upsilon, X_{0}, \Upsilon, \Upsilon_{\theta}\right)+\frac{2}{3} \varepsilon \dot{\psi}\left(R\left(\Upsilon, X_{0}, \eta, \Upsilon_{\theta}\right)+R\left(\eta, X_{0}, \Upsilon, \Upsilon_{\theta}\right)\right) \\
& +\frac{1}{3} \varepsilon \dot{\phi} R\left(\eta, \Upsilon, \Upsilon, \Upsilon_{\theta}\right)+\phi^{-2} \dot{\phi} \frac{\partial w}{\partial \theta}+\phi^{-1} \dot{\psi}<\frac{\partial \eta}{\partial x_{0}}, \Upsilon_{\theta}>_{e} \\
& +\varepsilon^{2} L(w, \eta)+Q(w, \eta)+O\left(\varepsilon^{3}\right) \\
\sigma_{3} & =2 \phi^{-1} w+\frac{1}{3} \varepsilon^{2} \phi^{2} R\left(\Upsilon, \Upsilon_{\theta}, \Upsilon, \Upsilon_{\theta}\right)+\frac{2}{3} \varepsilon \phi R\left(\Upsilon, \Upsilon_{\theta}, \eta, \Upsilon_{\theta}\right) \\
& +\varepsilon^{2} L(w, \eta)+Q(w, \eta)+O\left(\varepsilon^{3}\right) .
\end{aligned}\right.
$$

Notice that $\sigma_{1} \sigma_{3}-\sigma_{2}^{2}=\varepsilon^{2} L(w, \eta)+Q(w, \eta)+O\left(\varepsilon^{3}\right)$. We have

$$
\operatorname{det}\left(\begin{array}{ll}
g_{s s} & g_{s \theta} \\
g_{s \theta} & g_{\theta \theta}
\end{array}\right) \cong \varepsilon^{4} \phi^{4}\left(1+\sigma_{1}+\sigma_{3}\right)
$$

and

$$
\operatorname{det}\left(\begin{array}{ll}
g_{s s} & g_{s \theta} \\
g_{s \theta} & g_{\theta \theta}
\end{array}\right)^{-1} \cong \varepsilon^{-4} \phi^{-4}\left(1-\sigma_{1}-\sigma_{3}\right) .
$$

So the inverse matrix

$$
\begin{aligned}
\left(\begin{array}{ll}
g^{s s} & g^{s \theta} \\
g^{s \theta} & g^{\theta \theta}
\end{array}\right) & =\operatorname{det}\left(\begin{array}{cc}
g_{s s} & g_{s \theta} \\
g_{s \theta} & g_{\theta \theta}
\end{array}\right)^{-1}\left(\begin{array}{cc}
g_{\theta \theta} & -g_{s \theta} \\
-g_{s \theta} & g_{s s}
\end{array}\right) \\
& \cong \varepsilon^{-2} \phi^{-2}\left(\begin{array}{cc}
1-\sigma_{1} & -\sigma_{2} \\
-\sigma_{2} & 1-\sigma_{3}
\end{array}\right) .
\end{aligned}
$$

From this and Lemma 2.6 we can get 


\section{Lemma 2.7.}

$$
\left(\begin{array}{c}
a_{1} \\
a_{2}
\end{array}\right) \cong-\varepsilon^{-2} \phi^{-2}\left(\begin{array}{c}
<N_{0}, \partial_{s}> \\
<N_{0}, \partial_{\theta}>
\end{array}\right)=\left(\begin{array}{c}
O(\varepsilon)+\varepsilon^{-1} L(w, \eta)+\varepsilon^{-1} Q(w, \eta) \\
O(\varepsilon)+\varepsilon L(w, \eta)+\varepsilon^{-1} Q(w, \eta)
\end{array}\right) .
$$

Proof. From (8), we have

$$
\left(\begin{array}{l}
a_{1} \\
a_{2}
\end{array}\right)=\varepsilon^{-2} \phi^{-2}\left(\begin{array}{cc}
1-\sigma_{1} & -\sigma_{2} \\
-\sigma_{2} & 1-\sigma_{3}
\end{array}\right)\left(\begin{array}{c}
-<N_{0}, \partial_{s}> \\
-<N_{0}, \partial_{\theta}>
\end{array}\right) .
$$

By direct calculation, we have $\sigma_{i}\left\langle N_{0}, \partial_{s}\right\rangle$ and $\sigma_{i}\left\langle N_{0}, \partial_{\theta}\right\rangle$ are in fact $\varepsilon^{3} L(w, \eta)+\varepsilon Q(w, \eta)+O\left(\varepsilon^{4}\right)$. So we can get the conclusion.

From (8),

$$
k^{2}=<N_{0}, N_{0}>+a_{1}<N_{0}, \partial_{s}>+a_{2}<N_{0}, \partial_{\theta}>.
$$

From

$$
\left.\begin{array}{rl}
<N_{0}, N_{0}>= & <\frac{1}{\phi}\left(\dot{\phi} X_{0}-\dot{\psi} \Upsilon\right), \frac{1}{\phi}\left(\dot{\phi} X_{0}-\dot{\psi} \Upsilon\right)> \\
= & \frac{\dot{\phi}^{2}}{\phi^{2}}<X_{0}, X_{0}>+\frac{\dot{\psi}^{2}}{\phi^{2}}<\Upsilon, \Upsilon>-2 \frac{\dot{\phi} \dot{\psi}}{\phi^{2}}<X_{0}, \Upsilon> \\
= & 1+\varepsilon^{2} \dot{\phi}^{2} R\left(\Upsilon, X_{0}, \Upsilon, X_{0}\right)+2 \varepsilon \frac{\dot{\phi}^{2}}{\phi} R\left(\Upsilon, X_{0}, \eta, X_{0}\right) \\
& -\frac{4}{3} \varepsilon \frac{\dot{\phi} \dot{\psi}}{\phi} R\left(\Upsilon, X_{0}, \eta, \Upsilon\right)+\varepsilon^{2} L(w, \eta)+Q(w, \eta)+O\left(\varepsilon^{3}\right), \\
a_{1}<N_{0}, \partial_{s}>= & \left(\varepsilon E(\phi, \phi \psi, \psi)+\varepsilon^{-1} L(w, \eta)+\varepsilon^{-1} Q(w, \eta)\right) \varepsilon\left(-\frac{\dot{\psi}}{\phi} \frac{\partial w}{\partial s}\right. \\
& -\frac{\dot{\psi}^{2}}{\phi}<\frac{\partial \eta}{\partial x^{0}}, \Upsilon>e+\varepsilon^{2} \phi \dot{\phi} \dot{\psi} R\left(\Upsilon, X_{0}, \Upsilon, X_{0}\right) \\
& +2 \varepsilon \dot{\phi} \dot{\psi} R\left(\Upsilon, X_{0}, \eta, X_{0}\right)+\frac{2}{3}\left(\dot{\phi}^{2}-\dot{\psi}^{2}\right) R\left(\Upsilon, X_{0}, \eta, \Upsilon\right) \\
= & \left.+\varepsilon^{2} L(w, \eta)+Q(w, \eta)+O\left(\varepsilon^{3}\right)\right) \\
a_{2} L(w, \eta)+Q(w, \eta)+O\left(\varepsilon^{4}\right), \\
= & \left(O(\varepsilon)+\varepsilon L(w, \eta)+\varepsilon^{-1} Q(w, \eta)\right) \varepsilon\left(\frac{2}{3} \varepsilon^{2} \phi^{2} \dot{\phi} R\left(\Upsilon, X_{0}, \Upsilon, \Upsilon_{\theta}\right)\right. \\
+ & \frac{2}{3} \varepsilon \phi \dot{\phi}\left(R\left(\Upsilon, X_{0}, \eta, \Upsilon_{\theta}\right)+R\left(\eta, X_{0}, \Upsilon, \Upsilon_{\theta}\right)\right) \\
+ & \left.\varepsilon^{2} L(w, \eta)+Q(w, \eta)+O\left(\varepsilon^{3}\right)\right) \\
\varepsilon^{2} L(w, \eta)+Q(w, \eta)+O\left(\varepsilon^{4}\right),
\end{array}\right]
$$


we have

$$
\begin{aligned}
k^{2}= & 1+\varepsilon^{2} \dot{\phi}^{2} R\left(\Upsilon, X_{0}, \Upsilon, X_{0}\right)+2 \varepsilon \frac{\dot{\phi}^{2}}{\phi} R\left(\Upsilon, X_{0}, \eta, X_{0}\right)-\frac{4}{3} \varepsilon \frac{\dot{\phi} \dot{\psi}}{\phi} R\left(\Upsilon, X_{0}, \eta, \Upsilon\right) \\
& +\varepsilon^{2} L(w, \eta)+Q(w, \eta)+O\left(\varepsilon^{3}\right) .
\end{aligned}
$$

So we have

\section{Lemma 2.8.}

$$
\begin{aligned}
k= & 1+\frac{\varepsilon^{2}}{2} \dot{\phi}^{2} R\left(\Upsilon, X_{0}, \Upsilon, X_{0}\right)+\varepsilon \frac{\dot{\phi}^{2}}{\phi} R\left(\Upsilon, X_{0}, \eta, X_{0}\right)-\frac{2}{3} \varepsilon \frac{\dot{\phi} \dot{\psi}}{\phi} R\left(\Upsilon, X_{0}, \eta, \Upsilon\right) \\
& +\varepsilon^{2} L(w, \eta)+Q(w, \eta)+O\left(\varepsilon^{3}\right) .
\end{aligned}
$$

2.2.3 The second fundamental form and the mean curvature of $\mathcal{D}_{\phi_{\tau_{0}}, p_{0}, \varepsilon}(w, \eta)$.

Here we prove

$$
\begin{aligned}
H\left(\mathcal{D}_{\phi_{\tau_{0}}, p_{0}, \varepsilon}(w, \eta)\right)= & \frac{2}{\varepsilon}+\frac{1}{\varepsilon} \mathcal{L}_{S N \Gamma} w+<\mathcal{J} \eta, \Upsilon> \\
& +\varepsilon\left(F_{1}\left(\phi, \phi_{\psi}\right) \star R_{1}+F_{2}\left(\phi, \phi_{\psi}\right) \star R_{2}\right)+\varepsilon^{2} E\left(\phi, \phi_{\psi}, \psi\right) \\
& +F_{3}\left(\phi, \phi_{\psi}\right) \star R_{3}(\eta)+\varepsilon L(w, \eta)+\varepsilon^{-1} Q(w, \eta) .
\end{aligned}
$$

where

$$
\begin{aligned}
\mathcal{L}_{S N \Gamma} w=- & \frac{\dot{\psi}}{\phi^{3}}\left(\frac{\partial^{2} w}{\partial s^{2}}+\frac{\partial^{2} w}{\partial \theta^{2}}\right)-2\left(\phi^{2}-\tau_{0}\right) \frac{\dot{\phi}}{\phi^{4}} \frac{\partial w}{\partial s}-\frac{\dot{\psi}}{\phi^{3}} w \\
<\mathcal{J} \eta, \Upsilon>=- & \frac{\dot{\psi}^{3}}{\phi^{3}}<\frac{\partial^{2} \eta}{\partial x_{0}^{2}}, \Upsilon>-\frac{1}{\varepsilon}\left(\frac{\dot{\psi} \ddot{\psi}}{\phi^{3}}+2\left(\phi^{2}-\tau_{0}\right) \frac{\dot{\phi} \dot{\psi}}{\phi^{4}}\right)<\frac{\partial \eta}{\partial x_{0}}, \Upsilon> \\
+ & \phi^{-2}\left(2 \dot{\phi} \ddot{\psi}+2 \frac{\dot{\phi}^{2} \dot{\psi}}{\phi}+\frac{\dot{\psi}^{3}}{\phi}-2 \frac{\dot{\psi}^{2}}{\phi}\left(\phi^{2}-\tau_{0}\right)-2 \phi \dot{\phi}^{2}\right) \\
\times & R\left(\Upsilon, X_{0}, \eta, X_{0}\right), \\
F_{1}\left(\phi, \phi_{\psi}\right) \star R_{1}= & \frac{1}{3} \dot{\psi} R\left(\Upsilon, \Upsilon_{\theta}, \Upsilon, \Upsilon_{\theta}\right) \\
& +\phi^{-2}\left(\phi \dot{\phi} \ddot{\psi}+2 \dot{\phi}^{2} \dot{\psi}+\dot{\psi}^{3}-\left(\phi^{2}-\tau_{0}\right) \dot{\psi}^{2}-\phi^{2} \dot{\phi}^{2}\right) \\
& \times R\left(\Upsilon, X_{0}, \Upsilon, X_{0}\right) \\
F_{2}\left(\phi, \phi_{\psi}\right) \star R_{2}= & \frac{2}{3} \dot{\phi} R\left(\Upsilon_{\theta}, X_{0}, \Upsilon, \Upsilon_{\theta}\right) \\
F_{3}\left(\phi, \phi_{\psi}\right) \star R_{3}(\eta)= & \phi^{-2}\left(\frac{2}{3} \dot{\phi} \ddot{\phi}+\frac{2}{3} \frac{\dot{\phi}^{3}}{\phi}-\frac{2}{3} \dot{\psi} \ddot{\psi}-\frac{4}{3}\left(\phi^{2}-\tau_{0}\right) \frac{\dot{\phi} \dot{\psi}}{\phi}-\frac{2}{3} \phi \dot{\phi}+\frac{4}{3} \phi \dot{\phi} \dot{\psi}\right) \\
& \times R\left(\Upsilon, X_{0}, \eta, \Upsilon\right)+\frac{2}{3} \phi^{-1} \dot{\phi} R\left(\Upsilon_{\theta}, X_{0}, \eta, \Upsilon_{\theta}\right) .
\end{aligned}
$$


For our later needs, we assume

$$
F_{4}\left(\phi, \phi_{\psi}\right)=\phi^{-2}\left(\frac{2}{3} \dot{\phi} \ddot{\phi}+\frac{2}{3} \frac{\dot{\phi}^{3}}{\phi}-\frac{2}{3} \dot{\psi} \ddot{\psi}-\frac{4}{3}\left(\phi^{2}-\tau_{0}\right) \frac{\dot{\phi} \dot{\psi}}{\phi}+\frac{4}{3} \phi \dot{\phi} \dot{\psi}\right) .
$$

Remark.

$$
\begin{cases}\Pi_{0}\left(R\left(\Upsilon_{\theta}, X_{0}, \Upsilon, \Upsilon_{\theta}\right)\right) & =0, \\ \Pi_{0}\left(R\left(\Upsilon, X_{0}, \eta, \Upsilon\right)-R\left(\Upsilon_{\theta}, X_{0}, \eta, \Upsilon_{\theta}\right)\right) & =0, \\ \Pi_{1}\left(R\left(\Upsilon, \Upsilon_{\theta}, \Upsilon, \Upsilon_{\theta}\right)\right) & =0, \\ \Pi_{1}\left(R\left(\Upsilon, X_{0}, \Upsilon, X_{0}\right)\right) & =0, \\ \Pi_{1}\left(R\left(\Upsilon, X_{0}, \eta, \Upsilon\right)\right) & =0, \\ \Pi_{1}\left(R\left(\Upsilon_{\theta}, X_{0}, \eta, \Upsilon_{\theta}\right)\right) & =0 .\end{cases}
$$

And from the second one,

$$
\Pi_{0}\left(F_{3}\left(\phi, \phi_{\psi}\right) \star R_{3}(\eta)\right)=F_{4}\left(\phi, \phi_{\psi}\right) R\left(\Upsilon, X_{0}, \eta, \Upsilon\right) .
$$

The proof of (13) is very long calculation, which can be found in Appendix A.

\section{Jacobi operator}

In this section we study the linear operators which appear in the expression of $H\left(\mathcal{D}_{\phi_{\tau_{0}}, p_{0}, \varepsilon}(w, \eta)\right)$.

\subsection{Basic properties}

Consider (14) (15). The operator $\mathcal{L}_{S N \Gamma}$ is conjugate to the Jacobi operator which corresponds to the second variation of the energy functional.

It is easy to see that

$$
\mathcal{L}_{S N \Gamma}: \quad C_{\varepsilon}^{2, \alpha}(S N \Gamma) \quad \mapsto C_{\varepsilon}^{0, \alpha}(S N \Gamma)
$$

is bounded uniformly in $\varepsilon$.

$$
\mathcal{J}: C_{x_{0}, \varepsilon}^{2, \alpha}(\Gamma, N \Gamma) \mapsto C_{\varepsilon}^{\alpha}(\Gamma, N \Gamma)
$$

and

$$
\|\mathcal{J}(\eta)\|_{C_{\varepsilon}^{\alpha}} \leq \frac{C}{\varepsilon}\|\eta\|_{C_{x_{0}, \varepsilon}^{2, \alpha}}
$$

We let

$$
\mathcal{L}_{0}=\left.\mathcal{L}_{S N \Gamma}\right|_{\Pi_{0}\left(C_{\varepsilon}^{2, \alpha}(S N \Gamma)\right)}, \tilde{\mathcal{L}}=\left.\mathcal{L}_{S N \Gamma}\right|_{\tilde{\Pi}\left(C_{\varepsilon}^{2, \alpha}(S N \Gamma)\right)} .
$$

We are going to study the mapping properties of $\tilde{\mathcal{L}}, \mathcal{J}, \mathcal{L}_{0}$ in three different modes, i.e. high mode, 1 st mode, 0th mode. 


\subsection{High mode}

In this mode, we are going to prove that

$$
\tilde{\mathcal{L}}: \tilde{\Pi} C_{\varepsilon}^{2, \alpha}(S N \Gamma) \rightarrow \tilde{\Pi} C_{\varepsilon}^{0, \alpha}(S N \Gamma)
$$

is an isomorphism whose inverse is bounded independent of $\varepsilon$.

First it is clear that

$$
\tilde{\mathcal{L}}\left(\tilde{\Pi} C_{\varepsilon}^{2, \alpha}(S N \Gamma)\right) \subseteq \tilde{\Pi} C_{\varepsilon}^{0, \alpha}(S N \Gamma) .
$$

From $\frac{\partial}{\partial s}=\varepsilon \dot{\psi} \frac{\partial}{\partial x_{0}}=\dot{\psi} \frac{\partial}{\partial \psi}$, we have, for $w, v \in \tilde{\Pi} W_{\varepsilon}^{1,2}(S N \Gamma)$

$$
\begin{aligned}
\tilde{\mathcal{L}} w & =-\frac{\dot{\psi}}{\phi^{3}}\left(\frac{\partial^{2} w}{\partial s^{2}}+\frac{\partial^{2} w}{\partial \theta^{2}}\right)-2\left(\phi^{2}-\tau_{0}\right) \frac{\dot{\phi}}{\phi^{4}} \frac{\partial w}{\partial s}-\frac{\dot{\psi}}{\phi^{3}} w \\
& =-\frac{\phi}{\dot{\psi}^{3}}\left(\frac{\dot{\psi}^{3}}{\phi^{2}} \frac{\partial}{\partial \psi}\left(\frac{\dot{\psi}^{3}}{\phi^{2}} \frac{\partial}{\partial \psi} w\right)+\frac{\dot{\psi}^{4}}{\phi^{4}} w+\frac{\dot{\psi}^{4}}{\phi^{4}} \frac{\partial^{2} w}{\partial \theta^{2}}\right)
\end{aligned}
$$

Consider the bounded bilinear functional $B: \tilde{\Pi} C_{\varepsilon}^{2, \alpha}(S N \Gamma) \times \tilde{\Pi} C_{\varepsilon}^{2, \alpha}(S N \Gamma) \rightarrow \mathbb{R}$ defined by

$$
B(v, w)=\int_{S N \Gamma}(v(\phi \tilde{\mathcal{L}}) w) d \theta d \psi
$$

We have, for some positive constant $C\left(\tau_{0}\right)$ which only depends on $\tau_{0}$,

$$
\begin{aligned}
B(w, w) & =\int_{S N \Gamma}\left(\frac{\dot{\psi}^{3}}{\phi^{2}}\left|\frac{\partial}{\partial \psi} w\right|^{2}-\frac{\dot{\psi}}{\phi^{2}} w^{2}+\frac{\dot{\psi}}{\phi^{2}}\left|\frac{\partial w}{\partial \theta}\right|^{2}\right) d \theta d \psi \\
& \geq C\left(\tau_{0}\right) \int_{S N \Gamma}\left(\left|\frac{\partial w}{\partial \psi}\right|^{2}+\left|\frac{\partial w}{\partial \theta}\right|^{2}+|w|^{2}\right) d \theta d \psi
\end{aligned}
$$

The inequality holds because for $w \in \tilde{\Pi} W_{\varepsilon}^{1,2}(S N \Gamma)$, we have

$$
\int_{S N \Gamma \cap\left\{\psi=\psi_{0}\right\}}\left|\frac{\partial w}{\partial \theta}\right|^{2} d \theta \geq 4 \int_{S N \Gamma \cap\left\{\psi=\psi_{0}\right\}}|w|^{2} d \theta
$$

for every $\psi_{0}$.

From 17 and the Lax-Milgram theorem we know $\phi \tilde{\mathcal{L}}$ is invertible and

$$
\|w\|_{W_{\varepsilon}^{1,2}} \leq C\left(\tau_{0}\right)\|\phi \tilde{\mathcal{L}} w\|_{W_{\varepsilon}^{-1,2}} .
$$

And from standard regularity theory of elliptic PDE we can get

$$
\|w\|_{C_{\varepsilon}^{2, \alpha}} \leq C\left(\tau_{0}\right)\|\phi \tilde{\mathcal{L}} w\|_{C_{\varepsilon}^{0, \alpha}} \leq C\left(\tau_{0}\right)\|\tilde{\mathcal{L}} w\|_{C_{\varepsilon}^{0, \alpha}} .
$$

\subsection{1st mode}

In this mode, we are going to prove that

$$
\mathcal{J} \eta: C_{x_{0}, \varepsilon}^{2, \alpha}(\Gamma, N \Gamma) \rightarrow C_{\varepsilon}^{\alpha}(\Gamma, N \Gamma)
$$

is invertible and the inverse is independent of $\varepsilon$. 


\subsubsection{Some preparations}

First we need to find the relationship between the operator $\mathcal{J}$ and the Jacobi operator $\mathcal{J}_{A}$ of the geodesic. Notice that

$$
\begin{aligned}
\frac{\dot{\psi}^{3}}{\phi} \mathcal{J} \eta= & -\frac{\dot{\psi}^{3}}{\phi^{2}} \frac{\partial}{\partial x_{0}}\left(\frac{\dot{\psi}^{3}}{\phi^{2}} \frac{\partial \eta}{\partial x_{0}}\right) \\
& -\frac{\dot{\psi}^{3}}{\phi^{3}}\left(2 \dot{\phi} \ddot{\psi}+2 \frac{\dot{\phi}^{2} \dot{\psi}}{\phi}+\frac{\dot{\psi}^{3}}{\phi}-2 \frac{\dot{\psi}^{2}}{\phi}\left(\phi^{2}-\tau_{0}\right)-2 \phi \dot{\phi}^{2}\right) R\left(\eta, X_{0}\right) X_{0} .
\end{aligned}
$$

We define $y_{0}$ by

$$
\begin{cases}d y_{0} & =\frac{\phi^{2}}{\dot{\psi}^{3}} d x_{0}, \\ y_{0}(0) & =0 .\end{cases}
$$

Then $d y_{0}=\frac{\phi^{2}}{\dot{\psi}^{3}} d x_{0}$ and $\frac{\dot{\psi}^{3}}{\phi^{2}} \frac{\partial}{\partial x_{0}}=\frac{\partial}{\partial y_{0}}$. We know $x_{0}=\varepsilon \psi$. So the period of $\phi$ and $\psi$ or the derivatives of them (in $x_{0}$ coordinate) have period of order $\varepsilon$. So coefficients such as $\frac{\phi^{2}}{\dot{\psi}^{3}}$ and $\frac{\dot{\psi}^{3}}{\phi^{3}}\left(2 \dot{\phi} \ddot{\psi}+2 \frac{\dot{\phi}^{2} \dot{\psi}}{\phi}+\frac{\dot{\psi}^{3}}{\phi}-2 \frac{\dot{\psi}^{2}}{\phi}\left(\phi^{2}-\tau_{0}\right)-2 \phi \dot{\phi}^{2}\right)$ are highly oscillating in $x_{0}$ coordinate. To understand the mean value of the coefficients in the right way is the key to understand the operator $\mathcal{J}$.

Suppose $\psi \in\left[a_{1}, b_{1}\right]$ is one period of $\phi$. Suppose

$$
\frac{\int_{a_{1}}^{b_{1}} \frac{\phi^{2}}{\dot{\psi}^{3}} d \psi}{\int_{a_{1}}^{b_{1}} d \psi}=I_{1} .
$$

$I_{1}$ is approximately the ratio of the length of $y_{0}$ and that of $x_{0}$. In some sense $d y_{0} \cong I_{1} d x_{0}$. We have

$$
\frac{\dot{\psi}^{3}}{\phi} \mathcal{J} \eta=-\left.\frac{\partial^{2} \eta}{\partial y_{0}^{2}}\right|_{y_{0}\left(x_{0}\right)}-\left.\Psi_{1}\left(\phi, \phi_{\psi}\right) R\left(\eta, X_{0}\right) X_{0}\right|_{x_{0}}
$$

where

$$
\Psi_{1}\left(\phi, \phi_{\psi}\right)=\frac{\dot{\psi}^{3}}{\phi^{3}}\left(2 \dot{\phi} \ddot{\psi}+2 \frac{\dot{\phi}^{2} \dot{\psi}}{\phi}+\frac{\dot{\psi}^{3}}{\phi}-2 \frac{\dot{\psi}^{2}}{\phi}\left(\phi^{2}-\tau_{0}\right)-2 \phi \dot{\phi}^{2}\right) .
$$

Now we need the average of $\Psi_{1}\left(\phi, \phi_{\psi}\right)$ in the coordinate $y_{0}$. Note that $d y_{0}=$ $\frac{\phi^{2}}{\dot{\psi}^{3}} d x_{0}=\varepsilon \frac{\phi^{2}}{\dot{\psi}^{3}} d \psi$. If we assume $y_{0}\left(a_{1}\right)=y_{1}, y_{0}\left(b_{1}\right)=y_{2}$, then we have

$$
\begin{aligned}
& \frac{\int_{y_{1}}^{y_{2}} \Psi_{1}\left(\phi, \phi_{\psi}\right) d y_{0}}{\int_{y_{1}}^{y_{2}} d y_{0}} \\
= & \frac{\int_{a_{1}}^{b_{1}} \frac{1}{\phi}\left(2 \dot{\phi} \ddot{\psi}+2 \frac{\dot{\phi}^{2} \dot{\psi}}{\phi}+\frac{\dot{\psi}^{3}}{\phi}-2 \frac{\dot{\psi}^{2}}{\phi}\left(\phi^{2}-\tau_{0}\right)-2 \phi \dot{\phi}^{2}\right) d \psi}{\int_{a_{1}}^{b_{1}} \frac{\phi^{2}}{\dot{\psi}^{3}} d \psi} \\
= & I_{2} .
\end{aligned}
$$


This indicates that in some sense

$$
\begin{aligned}
& -\frac{\partial^{2} \eta}{\partial y_{0}^{2}}-\Psi_{1}\left(\phi, \phi_{\psi}\right) R\left(\eta, X_{0}\right) X_{0} \\
\cong & -\left.\frac{\partial^{2} \eta}{\partial y_{0}^{2}}\right|_{y_{0}}-\left.I_{2} R\left(\eta, X_{0}\right) X_{0}\right|_{x_{0}} .
\end{aligned}
$$

But $\frac{\partial}{\partial y_{0}} \cong I_{1}^{-1} \frac{\partial}{\partial x_{0}}$. So

$$
-\left.\frac{\partial^{2} \eta}{\partial y_{0}^{2}}\right|_{y_{0}}-\left.I_{2} R\left(\eta, X_{0}\right) X_{0}\right|_{x_{0}} \cong-\left.I_{1}^{-2}\left(\left.\frac{\partial^{2} \eta}{\partial x_{0}^{2}}\right|_{x_{0}}+I_{1}^{2} I_{2} R\left(\eta, X_{0}\right) X_{0}\right)\right|_{x_{0}} .
$$

So if

$$
I_{1}^{2} I_{2}=1,
$$

we will have the chance to unearth the Jacobi operator of the geodesic $\mathcal{J}_{A}$. Fortunately, it is true. Due to easy calculation, it is equivalent to the following lemma

Lemma 3.1. ("average 1" lemma)

$$
\begin{aligned}
& \int_{a_{1}}^{b_{1}} \frac{1}{\phi}\left(2 \dot{\phi} \ddot{\psi}+2 \frac{\dot{\phi}^{2} \dot{\psi}}{\phi}+\frac{\dot{\psi}^{3}}{\phi}-2 \frac{\dot{\psi}^{2}}{\phi}\left(\phi^{2}-\tau_{0}\right)-2 \phi \dot{\phi}^{2}\right) d \psi \cdot \int_{a_{1}}^{b_{1}} \frac{\phi^{2}}{\dot{\psi}^{3}} d \psi \\
= & \left(b_{1}-a_{1}\right)^{2} .
\end{aligned}
$$

The proof of this lemma is direct calculations. The proof can be found in Appendix B

Let $\tilde{y}=I_{1}^{-1} y_{0}$. First we define a map $\mathfrak{F}: \Gamma \rightarrow \Gamma . \mathfrak{F}(p)=q$ if and only if $x_{0}(p)=\tilde{y}(q)$. The following graph illustrate the definition of $\mathfrak{F}$.

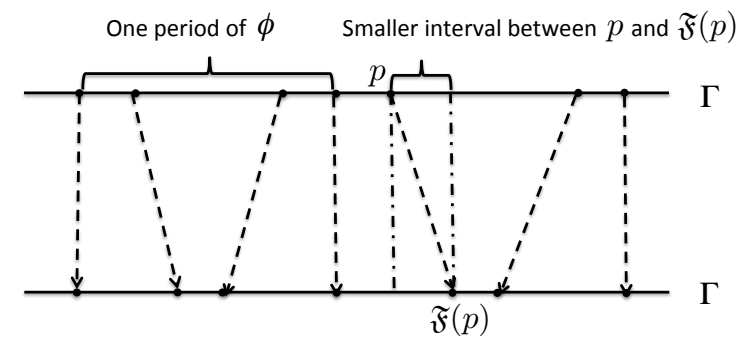

Let $P_{p}^{\widetilde{F}(p)}:\left.\left.(N \Gamma)^{*} \otimes N \Gamma\right|_{p} \rightarrow(N \Gamma)^{*} \otimes N \Gamma\right|_{\mathfrak{F}(p)}$ be defined by parallel translating any element of $\left.(N \Gamma)^{*} \otimes N \Gamma\right|_{p}$ to the space $\left.(N \Gamma)^{*} \otimes N \Gamma\right|_{\mathfrak{F}(p)}$, along the smaller interval between $p$ and $\mathfrak{F}(p)$. Let $\tilde{\mathcal{J}}_{A}=-\frac{\partial^{2}}{\partial y_{0}^{2}}-I_{2}\left(P_{p}^{\mathfrak{F}(p)} R\left(\cdot, X_{0}\right) X_{0}\right)$.

Lemma 3.2. $I_{1}^{2} \tilde{\mathcal{J}}_{A}$ is conjugate to $\mathcal{J}_{A}$. So $\tilde{\mathcal{J}}_{A}$ is invertible and

$$
\|\eta\|_{C_{y_{0}}^{2}} \leq C\left\|\tilde{\mathcal{J}}_{A} \eta\right\|_{C^{0}}
$$


Proof. Let $\eta_{1}$ be a smooth section of $N \Gamma$. Define $\left(\eta_{1} \circ \mathfrak{F}\right)(p) \in N \Gamma(p)$ by parallel translating $\eta_{1}$ from $\mathfrak{F}(p)$ to $p$ along the smaller interval between them. For another section $\eta_{2}, \eta_{2} \circ \mathfrak{F}^{-1}$ can be defined similarly.

$$
\begin{aligned}
I_{1}^{2} \tilde{\mathcal{J}}_{A}\left(\eta_{1}\right)(\mathfrak{F}(p)) & =-\frac{\partial^{2} \eta_{1}(\mathfrak{F}(p))}{\partial \tilde{y}^{2}}-\left(P_{p}^{\widetilde{F}(p)} R\left(\cdot, X_{0}\right) X_{0}\right)\left(\eta_{1}(\mathfrak{F}(p))\right) \\
& =-\frac{\partial^{2}\left(\eta_{1} \circ \mathfrak{F}\right)(p)}{\partial x_{0}^{2}}-R\left(\left(\eta_{1} \circ \mathfrak{F}\right)(p), X_{0}\right) X_{0} .
\end{aligned}
$$

So

$$
I_{1}^{2} \tilde{\mathcal{J}}_{A}\left(\eta_{1}\right)(q)=\left(-\frac{\partial^{2}\left(\eta_{1} \circ \mathfrak{F}\right)(p)}{\partial x_{0}^{2}}-R\left(\left(\eta_{1} \circ \mathfrak{F}\right)(p), X_{0}\right) X_{0}\right) \circ \mathfrak{F}^{-1}(q) .
$$

It is obvious that $C^{-1}\|\eta \circ \mathfrak{F}\|_{C_{x_{0}}^{2}} \leq\|\eta\|_{C_{y_{0}}^{2}} \leq C\|\eta \circ \mathfrak{F}\|_{C_{x_{0}}^{2}}$. So we can prove 20p.

Definition 3. The Green operator $G\left(y_{0}, z_{0}\right):\left.\left.N \Gamma\right|_{z_{0}} \rightarrow N \Gamma\right|_{y_{0}}$ is a linear map for given $y_{0}, z_{0}$ which satisfies

1. For fixed $z_{0}$, and $\left.\nu \in N \Gamma\right|_{z_{0}}, \tilde{\mathcal{J}}_{A}\left(G\left(y_{0}, z_{0}\right) \nu\right)=0, y_{0} \neq z_{0}$, where $\tilde{\mathcal{J}}_{A}$ acts on $y_{0}$.

2. For fixed $z_{0},\left.\nu \in N \Gamma\right|_{z_{0}}, G\left(y_{0}, z_{0}\right) \nu$ can be extended to be continuous at $y_{0}=z_{0}$ and $\frac{\partial G\left(z_{0}^{+}, z_{0}\right) \nu}{\partial y_{0}}-\frac{\partial G\left(z_{0}^{-}, z_{0}\right) \nu}{\partial y_{0}}=\nu$.

It is obvious that $G_{z_{0}}\left(y_{0}, z_{0}\right)$ and $G_{y_{0}}\left(y_{0}, z_{0}\right)$ make sense in light of the notion of connection of $N \Gamma$.

Lemma 3.3. The Green operator exists. If $\tilde{\mathcal{J}}_{A} \eta=f$, then

$$
\eta\left(y_{0}\right)=\int_{\Gamma} G\left(y_{0}, z_{0}\right) f\left(z_{0}\right) d z_{0} .
$$

Moreover, $G\left(y_{0}, z_{0}\right)$ satisfies that

1. For fixed $z_{0}$, let $\left.\nu \in N \Gamma\right|_{z_{0}},|\nu|=1$. Then $G\left(y_{0}, z_{0}\right) \nu \in C_{y_{0}}^{\infty}\left(\Gamma \backslash\left\{z_{0}\right\}, N \Gamma\right)$ and can be extended to be in $C_{y_{0}}^{0,1}(\Gamma, N \Gamma)$ and the $C_{y_{0}}^{0,1}$ norm is bounded independent of $z_{0}$ and $\nu$.

2. Let $\left.\nu \in N \Gamma\right|_{z_{0}},|\nu|=1$. $G_{z_{0}}\left(y_{0}, z_{0}\right) \nu, G_{z_{0} y_{0}}\left(y_{0}, z_{0}\right) \nu, y_{0} \neq z_{0}$ are bounded independent of $y_{0}, z_{0}$.

Proof. For fixed $z_{0}$, we choose orthonormal basis $E_{1},\left.E_{2} \in N \Gamma\right|_{z_{0}}$. Let $\mathfrak{J}$ be the Jacobi field which is orthonormal to $\gamma$, with $\mathfrak{J}\left(z_{0}^{+}\right)=a E_{1}+b E_{2}, \mathfrak{J}^{\prime}\left(z_{0}^{+}\right)=c E_{1}+$ $d E_{2}$. We extend $\mathfrak{J}$ along $\gamma$ to $z_{0}^{-}$. And It is obvious that there is a $4 \times 4$ matrix $A\left(z_{0}\right)$ which does not depends on $\mathfrak{J}$, such that under the basis $E_{1}, E_{2}$

$$
\left(\begin{array}{c}
\mathfrak{J}\left(z_{0}^{-}\right) \\
\mathfrak{J}^{\prime}\left(z_{0}^{-}\right)
\end{array}\right)=A\left(z_{0}\right)\left(\begin{array}{c}
a \\
b \\
c \\
d
\end{array}\right) .
$$


As $\tilde{\mathcal{J}}_{A}$ is nondegenerate, 1 is not the eigenvalue of $A\left(z_{0}\right)$. So we can solve

$$
\left(A\left(z_{0}\right)-I\right)\left(\begin{array}{c}
a_{1} \\
b_{1} \\
c_{1} \\
d_{1}
\end{array}\right)=\left(\begin{array}{c}
0 \\
0 \\
-1 \\
0
\end{array}\right) \text { and }\left(A\left(z_{0}\right)-I\right)\left(\begin{array}{c}
a_{2} \\
b_{2} \\
c_{2} \\
d_{2}
\end{array}\right)=\left(\begin{array}{c}
0 \\
0 \\
0 \\
-1
\end{array}\right) .
$$

We let the Jacobi field $\mathfrak{J}_{i}^{z_{0}}\left(y_{0}\right)$ satisfy $\mathfrak{J}_{i}^{z_{0}}\left(z_{0}^{+}\right)=a_{i} E_{1}+b_{i} E_{2}, \frac{\partial}{\partial y_{0}} \mathfrak{J}_{i}^{z_{0}}\left(z_{0}^{+}\right)=c_{i} E_{1}+$ $d_{i} E_{2}$. So $\mathfrak{J}_{i}^{z_{0}}\left(z_{0}^{+}\right)=\mathfrak{J}_{i}^{z_{0}}\left(z_{0}^{-}\right)$and $\frac{\partial}{\partial y_{0}} \mathfrak{J}_{i}^{z_{0}}\left(z_{0}^{+}\right)=\frac{\partial}{\partial y_{0}} \mathfrak{J}_{i}^{z_{0}}\left(z_{0}^{-}\right)+E_{i} . G\left(y_{0}, z_{0}\right)$ is defined by $G\left(y_{0}, z_{0}\right) E_{i}=\mathfrak{J}_{i}^{z_{0}}\left(y_{0}\right)$. One can check that $G\left(y_{0}, z_{0}\right)$ is the Green operator and it is the inverse of $\tilde{\mathcal{J}}_{A}$.

Now we prove the first item. It is obvious that $\mathfrak{J}_{i}^{z_{0}}\left(y_{0}\right)$ is a continuous function of $\left(y_{0}, z_{0}\right) \in \Gamma \times \Gamma$. So it is bounded. From the Jacobi equation, the $\frac{\partial^{2}}{\partial y_{0}^{2}} \mathfrak{J}_{i}^{z_{0}}\left(y_{0}\right)$ is uniformly bounded when $y_{0} \neq z_{0}$. As $\left(A\left(z_{0}\right)-I\right)^{-1}$ is uniformly bounded, we know $\frac{\partial}{\partial y_{0}} \mathfrak{J}_{i}^{z_{0}}\left(z_{0}^{ \pm}\right)$is uniformly bounded. So $\frac{\partial}{\partial y_{0}} \mathfrak{J}_{i}^{z_{0}}\left(y_{0}\right)$ is uniformly bounded in $\Gamma \times \Gamma \backslash\{(p, p)\}$. So the first item is clear.

For the second item, first we know that for fixed $y_{0} G\left(y_{0}, z_{0}\right)$ is smooth in $z_{0} \neq$ $y_{0}$ and $G_{z_{0}}\left(y_{0}, z_{0}\right) E_{i}$ is also a Jacobi field. As $\frac{d}{d z_{0}}\left(G\left(z_{0}, z_{0}\right) E_{i}\right)=\frac{d}{d z_{0}} \mathfrak{J}_{i}^{z_{0}}\left(z_{0}\right)$ is uniformly bounded and $\frac{d}{d z_{0}}\left(G\left(z_{0}, z_{0}\right) E_{i}\right)=G_{y_{0}}\left(z_{0}^{ \pm}, z_{0}\right) E_{i}+G_{z_{0}}\left(z_{0}^{ \pm}, z_{0}\right) E_{i}$, we know $G_{z_{0}}\left(z_{0}^{ \pm}, z_{0}\right) E_{i}$ is uniformly bounded. Again as $\frac{d}{d z_{0}}\left(G_{y_{0}}\left(z_{0}, z_{0}\right) E_{i}\right)=\frac{d}{d z_{0}} \frac{\partial}{\partial y_{0}} \mathfrak{J}_{i}^{z_{0}}\left(z_{0}\right)$ is bounded, and $\frac{d}{d z_{0}}\left(G_{y_{0}}\left(z_{0}, z_{0}\right) E_{i}\right)=G_{y_{0} y_{0}}\left(z_{0}^{ \pm}, z_{0}\right) E_{i}+G_{z_{0} y_{0}}\left(z_{0}^{ \pm}, z_{0}\right) E_{i}$, we know $G_{z_{0} y_{0}}\left(z_{0}^{ \pm}, z_{0}\right) E_{i}$ is uniformly bounded. So $G_{z_{0}}\left(y_{0}, z_{0}\right) E_{i}$ is uniformly bounded from the comparison theorem of Jacobi fields. Also $G_{z_{0} y_{0}}\left(y_{0}, z_{0}\right) E_{i}$ is uniformly bounded from the Jacobi equation.

Lemma 3.4. Suppose that $F\left(\phi, \phi_{\psi}\right)$ is a smooth function of $\phi$ and $\phi_{\psi}$ and it has the cancellation property in $y_{0}$ coordinate, i.e.

$$
\int_{y_{1}}^{y_{2}} F\left(\phi, \phi_{\psi}\right) d y_{0}=0
$$

where $\left[y_{1}, y_{2}\right]$ is one period of $\phi\left(y_{0}\right)$. Let $R$ be a $C_{x_{0}}^{1}$ section of $N \Gamma$. Then

$$
h\left(y_{0}\right)=\int_{\Gamma} G\left(y_{0}, z_{0}\right) R\left(z_{0}\right) F\left(\phi, \phi_{\psi}\right)\left(z_{0}\right) d z_{0}
$$

satisfies

$$
\left\|h\left(y_{0}\right)\right\|_{C_{y_{0}}^{1}} \leq C \varepsilon\|R\|_{C_{y_{0}}^{1}} \leq C \varepsilon\|R\|_{C_{x_{0}}^{1}}
$$

where $C$ depends on $\tau_{0}$.

Proof. Suppose $\chi\left(y_{0}\right)$ is the primitive function of $F\left(\phi, \phi_{\psi}\right)$ in $y_{0}$ coordinate. Because $F\left(\phi, \phi_{\psi}\right)$ has cancellation property, $\chi\left(y_{0}\right)$ is a global periodic function on $\Gamma$. We may add a constant to $\chi\left(y_{0}\right)$ such that it also has cancellation property in $y_{0}$ coordinate. It is easy verified that

$$
\left\|\chi\left(y_{0}\right)\right\|_{C^{0}} \leq C\left(\tau_{0}\right) \varepsilon
$$


We have

$$
h\left(y_{0}\right)=-\int_{\Gamma} \chi\left(z_{0}\right)\left(G_{z_{0}} R+G R_{z_{0}}\right) d z_{0}
$$

and

$$
\begin{aligned}
h^{\prime}\left(y_{0}\right) & =\int_{\Gamma} G_{y_{0}} R\left(z_{0}\right) F\left(\phi, \phi_{\psi}\right)\left(z_{0}\right) d z_{0} \\
& =\int_{0}^{L_{\Gamma}} G_{y_{0}}\left(y_{0}, y_{0}+z\right) R\left(y_{0}+z\right) F\left(\phi, \phi_{\psi}\right)\left(y_{0}+z\right) d z \\
& =\left.G_{y_{0}}\left(y_{0}, y_{0}+z\right) R\left(y_{0}+z\right) \chi\left(y_{0}+z\right)\right|_{0} ^{L_{\Gamma}}-\int_{0}^{L_{\Gamma}} \chi\left(y_{0}+z\right)\left(G_{y_{0} z} R+G_{y_{0}} R_{z}\right) d z .
\end{aligned}
$$

From $\left|R_{z_{0}}\right| \leq C\left|R_{x_{0}}\right| \leq C, 22$ and Lemma 3.3 we can prove this lemma.

\subsubsection{The main theorem of 1st mode}

Now we can discuss the operator $\mathcal{J}$. First it is clear that, there is a map $P_{1}$ : $L^{2}(S N \Gamma) \rightarrow L^{2}(N \Gamma)$ such that for any $f \in L^{2}(S N \Gamma)$

$$
\Pi_{1}(f)=<P_{1}(f), \Upsilon>\text {. }
$$

We have

Theorem 3.5. There is $\delta>0$ such that when $0<\varepsilon<\delta$, we have

1. For each $f \in C_{\varepsilon}^{\alpha}(N \Gamma)$, there exists a unique $\eta \in C_{x_{0}, \varepsilon}^{2, \alpha}(N \Gamma)$ such that

$$
\mathcal{J} \eta=f
$$

and for some uniform $C$ which does not depend on $\varepsilon$

$$
\|\eta\|_{C_{x_{0}, \varepsilon}^{2, \alpha}} \leq C\|f\|_{C_{\varepsilon}^{\alpha}}
$$

2. The equation

$$
\mathcal{J} \eta=-\frac{2}{3} \varepsilon \dot{\phi} P_{1}\left(R\left(\Upsilon_{\theta}, X_{0}, \Upsilon, \Upsilon_{\theta}\right)\right)
$$

has a unique solution $\eta$ such that for some uniform $C$

$$
\|\eta\|_{C_{x_{0}, \varepsilon}^{2, \alpha}} \leq C \varepsilon^{2} .
$$

Proof. The proof of the first item. Consider

$$
\begin{aligned}
\frac{\dot{\psi}^{3}}{\phi} \mathcal{J} \eta(q)= & -\frac{\partial^{2} \eta}{\partial y_{0}^{2}}(q)-\left.\Psi_{1}\left(\phi, \phi_{\psi}\right) R\right|_{q}\left(\eta(q), X_{0}\right) X_{0} \\
= & \tilde{\mathcal{J}}_{A} \eta+\left(I_{2}-\left.\Psi_{1}\left(\phi, \phi_{\psi}\right)\right|_{q}\right)\left(P_{\mathfrak{F}^{-1}(q)}^{q} R\right)\left(\eta(q), X_{0}\right) X_{0} \\
& +\left.\Psi_{1}\left(\phi, \phi_{\psi}\right)\right|_{q}\left(\left(P_{\mathfrak{F}^{-1}(q)}^{q} R\right)-\left.R\right|_{q}\right)\left(\eta(q), X_{0}\right) X_{0} \\
= & \tilde{\mathcal{J}}_{A} \eta+\left(I_{2}-\left.\Psi_{1}\left(\phi, \phi_{\psi}\right)\right|_{q}\right)\left(P_{\mathfrak{F}^{-1}(q)}^{q} R\right)\left(\eta(q), X_{0}\right) X_{0}+O(\varepsilon) L(\eta) \\
= & \frac{\dot{\psi}^{3}}{\phi} f
\end{aligned}
$$


First we solve

$$
\tilde{\mathcal{J}}_{A} \eta_{1}=\frac{\dot{\psi}^{3}}{\phi} f
$$

From the invertibility of $\tilde{\mathcal{J}}_{A}$ and Lemma 3.3 we know there is one unique solution $\eta_{1}$

$$
\begin{aligned}
\left\|\eta_{1}\right\|_{C_{y_{0}}^{2}} & \leq\left\|\int_{\Gamma} G\left(y_{0}, z_{0}\right) \frac{\dot{\psi}^{3}}{\phi} f d z_{0}\right\|_{C^{0}} \\
& \leq\|f\|_{C^{0}} .
\end{aligned}
$$

Then for each $i \geq 1$ we solve

$$
\begin{aligned}
& \tilde{\mathcal{J}}_{A}\left(\eta_{i+1}-\eta_{i}\right)(q) \\
= & \left(\left.\Psi_{1}\left(\phi, \phi_{\psi}\right)\right|_{q}-I_{2}\right)\left(P_{\mathfrak{F}^{-1}(q)}^{q} R\right)\left(\eta_{i}(q)-\eta_{i-1}(q), X_{0}\right) X_{0}+O(\varepsilon) L\left(\eta_{i}-\eta_{i-1}\right)
\end{aligned}
$$

where $\eta_{0}=0$. From 19 we know $\left.\Psi_{1}\left(\phi, \phi_{\psi}\right)\right|_{x_{0}}-I_{2}$ has 0 average in one period in $y_{0}$ coordinate. From Lemma 3.4 , we know

$$
\left\|\eta_{i+1}-\eta_{i}\right\|_{C_{y_{0}}^{1}} \leq C \varepsilon\left\|\eta_{i}-\eta_{i-1}\right\|_{C_{y_{0}}^{1}}
$$

where $C$ depends on $\tau_{0}$ and the norm of the curvature. So we can choose $\delta$ such that $0<C \delta<\frac{1}{2}$. When $\varepsilon<\delta, 0<C \varepsilon<\frac{1}{2}$. So $\eta_{i}$ converges to some $\eta$ in $C_{y_{0}}^{1}$. From 25

$$
\left\|\eta_{i+1}-\eta_{i}\right\|_{C_{y_{0}}^{2}} \leq C\left\|\eta_{i}-\eta_{i-1}\right\|_{C_{y_{0}}^{1}}
$$

we know $\partial_{y_{0}}^{2} \eta_{i} \rightarrow \partial_{y_{0}}^{2} \eta$ in $C^{0}$ sense. So we get a solution for $\mathcal{J} \eta=f$ and we have the estimate

$$
\|\eta\|_{C_{y_{0}}^{1}} \leq\|f\|_{C^{0}}
$$

So we have

$$
\|\eta\|_{C_{x_{0}}^{1}} \leq\|f\|_{C^{0}}
$$

and hence from 23 .

$$
\|\eta\|_{C_{x_{0}, \varepsilon}^{2, \alpha}} \leq\|f\|_{C_{\varepsilon}^{\alpha}} .
$$

Now we prove the second item. In 24, we let $f=-\frac{2}{3} \varepsilon \dot{\phi} P_{1}\left(R\left(\Upsilon_{\theta}, X_{0}, \Upsilon, \Upsilon_{\theta}\right)\right)$. It is obvious that $\frac{\dot{\psi}^{3}}{\phi} \dot{\phi}$ has 0 average in one period. Apply Lemma 3.4 again, we can draw the conclusion.

\subsection{The sketch of the proof and main difficulties}

Now we are in a position to sketch the whole proof and state the main difficulities. It would be helpful to analyze the three modes together. Basically, we would like 
to use an iteration method to solve the following system.

$$
\left\{\begin{aligned}
\mathcal{L}_{0} w_{0}= & -\varepsilon^{2} F_{1}\left(\phi, \phi_{\psi}\right) \star \Pi_{0}\left(R_{1}\right)-\varepsilon \Pi_{0}(E) \\
& -\varepsilon F_{3}\left(\phi, \phi_{\psi}\right) \star \Pi_{0}\left(R_{3}(\eta)\right) \\
& -\varepsilon \Pi_{0}\left(\varepsilon L\left(w_{0}+\tilde{w}, \eta\right)+\varepsilon^{-1} Q\left(w_{0}+\tilde{w}, \eta\right)\right), \\
<\mathcal{J} \eta, \Upsilon>= & -\varepsilon F_{2}\left(\phi, \phi_{\psi}\right) \star \Pi_{1}\left(R_{2}\right)-\Pi_{1}(E) \\
& -\Pi_{1}\left(\varepsilon L\left(w_{0}+\tilde{w}, \eta\right)+\varepsilon^{-1} Q\left(w_{0}+\tilde{w}, \eta\right)\right), \\
\tilde{\mathcal{L}} \tilde{w}=\quad & -\varepsilon^{2} F_{1}\left(\phi, \phi_{\psi}\right) \star \tilde{\Pi}\left(R_{1}\right)-\varepsilon^{2} F_{2}\left(\phi, \phi_{\psi}\right) \star \tilde{\Pi}\left(R_{2}\right) \\
& -\varepsilon \tilde{\Pi}(E)-\varepsilon F_{3}\left(\phi, \phi_{\psi}\right) \star \tilde{\Pi}\left(R_{3}(\eta)\right) \\
& -\varepsilon \tilde{\Pi}\left(\varepsilon L\left(w_{0}+\tilde{w}, \eta\right)+\varepsilon^{-1} Q\left(w_{0}+\tilde{w}, \eta\right)\right) .
\end{aligned}\right.
$$

The two fundamental solutions for $\mathcal{L}_{0} w_{0}=0$ are $W_{1}=h_{1}\left(\tau_{0}\right) \phi_{\psi}$ and $W_{2}=$ $h_{2}\left(\tau_{0}\right) \psi \phi_{\psi}+v$, where $v$ is a periodic function. $W_{1}$ is periodic and $W_{2}$ has linear growth. $w_{0}$ can be expressed using $\mathcal{L}_{0} w_{0}$ by

$$
\begin{aligned}
w_{0}(\psi)= & c_{1} W_{1}(\psi)+c_{2} W_{2}(\psi) \\
& -\int_{0}^{\psi}\left(W_{2}(\psi) W_{1}(t)-W_{1}(\psi) W_{2}(t)\right) \sigma(t)^{-1}\left(1+\phi_{\psi}^{2}\right)^{-\frac{3}{2}} \mathcal{L}_{0} w_{0} d \psi,
\end{aligned}
$$

where $\sigma(\psi)=W_{2}^{\prime}(\psi) W_{1}(\psi)-W_{1}^{\prime}(\psi) W_{2}(\psi)$.

There are three difficulties in solving the above equation system.

1. The operator $\mathcal{L}_{0}$ has kernel $\frac{\dot{\phi}}{\dot{\psi}}=\phi_{\psi}$ which corresponds to the translation of the surface along the geodesic. So one may only solve it up to the kernel. We have to add one term $\varepsilon^{3} \omega \phi^{-1} \phi_{\psi}$ to the right hand side of the first equation. Once we have a solution $w_{0}$, which is smooth at any $p \in \Gamma$, we get infinite many solutions as we have freedom to choose $c_{1}$. We let $c_{1}=0$ to have a unique solution which satisfies $w_{0}^{\prime}(0)=0$. To get such a solution, first we adjust $\omega$ such that $w_{0}(0)=w_{0}\left(\frac{L_{\Gamma}}{\varepsilon}\right)$. Then we adjust $c_{2}$ such that $w_{0}^{\prime}\left(0^{+}\right)=w_{0}^{\prime}\left({\frac{L_{\Gamma}}{\varepsilon}}^{-}\right)$.

2. From 26, we generally expect $w_{0}$ to be of the size $\frac{1}{\varepsilon^{2}} \mathcal{L}_{0} w_{0}$. Then as $-\varepsilon^{2} F_{1}\left(\phi, \phi_{\psi}\right) \star \Pi_{0}\left(R_{1}\right)$ is of size $\varepsilon^{2}$ and there is one term $\left(\partial_{s} w\right)\left(\partial_{s}^{2} w\right)$ in $\Pi_{0}(Q(w, \eta))$, it is impossible to solve the first equation using an iteration method, even given $\tilde{w}=0$ and $\eta=0$. Actually there is a cancellation property for $-\varepsilon^{2} F_{1}\left(\phi, \phi_{\psi}\right) \star \Pi_{0}\left(R_{1}\right)$ so that it looks as if it has order $O\left(\varepsilon^{3}\right)$. Even though, the iteration still breaks down.

3. The term $F_{3}\left(\phi, \phi_{\psi}\right) \star R_{3}(\eta)$ also causes problem because there is a term of the type $\frac{1}{s} w \cdot \partial_{x_{0}} \eta$ in $\Pi_{1}(Q(w, \eta))$. Once $w_{0}$ has a variation of $O(\varepsilon)$. From Theorem 3.5, $\eta$ will have a variation of size $O\left(\varepsilon^{2}\right)$. Now if we go back to 0th mode, $w_{0}$ will again have a variation of size $O(\varepsilon)$. So even given $\tilde{w}=0$, it is impossible to solve the first two equations together.

The second difficulty appears because of the shortcoming of

$$
\begin{aligned}
\mathcal{L}_{0} w_{0}= & -\varepsilon^{2} F_{1}\left(\phi, \phi_{\psi}\right) \star \Pi_{0}\left(R_{1}\right)-\varepsilon \Pi_{0}(E)-\varepsilon F_{3}\left(\phi, \phi_{\psi}\right) \star \Pi_{0}\left(R_{3}(\eta)\right) \\
& -\varepsilon \Pi_{0}\left(\varepsilon L\left(w_{0}+\tilde{w}, \eta\right)+\varepsilon^{-1} Q\left(w_{0}+\tilde{w}, \eta\right)\right),
\end{aligned}
$$


that is, $Q\left(w_{0}+\tilde{w}, \eta\right)$ involves special structures, which are hard to analyze and use. Now we consider 0 th mode in a nonlinear way. Notice that if $\phi_{\tau_{0}}$ satisfies (2),

$$
\begin{aligned}
\Pi_{0}\left(H\left(\mathcal{D}_{\phi_{\tau_{0}}, p_{0}, \varepsilon}(w, \eta)\right)-\frac{2}{\varepsilon}\right)= & \varepsilon F_{1}\left(\phi_{\tau_{0}},\left(\phi_{\tau_{0}}\right)_{\psi}\right) \star \Pi_{0}\left(R_{1}\right)+\Pi_{0}(E) \\
& +F_{3}\left(\phi_{\tau_{0}},\left(\phi_{\tau_{0}}\right)_{\psi}\right) \star \Pi_{0}\left(R_{3}(\eta)\right) \\
& +\Pi_{0}\left(\varepsilon L\left(w_{0}+\tilde{w}, \eta\right)+\varepsilon^{-1} Q\left(w_{0}+\tilde{w}, \eta\right)\right) .
\end{aligned}
$$

The key idea is we substitute the solution $\phi_{\xi, \mu}(\psi)$ of the following nonlinear ODE for $\phi_{\tau_{0}}$. We let $\psi\left(p_{0}\right)=0, \bmod \frac{L_{\Gamma}}{\varepsilon}$. Consider

$$
\begin{cases}\phi_{\psi \psi} & -\phi^{-1}\left(1+\phi_{\psi}^{2}\right)+(2+\rho)\left(1+\phi_{\psi}^{2}\right)^{\frac{3}{2}}=0, \\ \phi_{\psi}(0) & =\phi_{\psi}\left(\frac{L_{\Gamma}}{\varepsilon}\right)=0, \\ \phi(0) & =\phi\left(\frac{L_{\Gamma}}{\varepsilon}\right)=\frac{1-\sqrt{1-4 \tau(0)}}{2}, \\ \rho & =-\varepsilon^{2} F_{1}\left(\phi, \phi_{\psi}\right) \star \Pi_{0}\left(R_{1}\right)+\varepsilon F_{4}\left(\phi, \phi_{\psi}\right) \xi\left(x_{0}\right) \\ & +\varepsilon^{3} \mu(\psi)+\varepsilon^{3} \omega \phi^{-1} \phi_{\psi},\end{cases}
$$

where $\tau(0)=\tau(\phi(0), 0)$ and

$$
\|\xi\|_{C_{x_{0}}^{1}} \leq C_{1} \varepsilon^{2},\|\mu\|_{C_{\varepsilon}^{\alpha}} \leq C_{2},|\omega| \leq C_{3},\left|\phi(0)-\frac{1-\sqrt{1-4 \tau_{0}}}{2}\right| \leq C\left(\tau_{0}\right) \varepsilon,
$$

where

$$
C\left(\tau_{0}\right)=\frac{1}{10} \min \left\{\frac{\sqrt{1-4 \tau_{0}}}{2}, \frac{1-\sqrt{1-4 \tau_{0}}}{2}, \frac{1}{4}-\tau_{0}, \tau_{0}\right\} .
$$

Among $\xi, \mu, \omega, \tau(0)$, only $\xi, \mu$ have freedom because this equation is overdetermined. Once we have prescribed $\xi, \mu$, we need to adjust $\omega$ and $\tau(0)$ to get a global smooth solution. Theorem 3.7 contains the main results of 0th mode, one may refer to which for the existence, uniqueness and estimates of the solution $\phi_{\xi, \mu}$ to (27).

In Subsection 4.1 we calculate $H\left(\mathcal{D}_{\phi_{\xi, \mu}, p_{0}, \varepsilon}(\tilde{w}, \eta)\right)$ instead, where $\Pi_{0}(\tilde{w})=0$. The expression for $H\left(\mathcal{D}_{\phi_{\xi, \mu}, p_{0}, \varepsilon}(\tilde{w}, \eta)\right)$ is 50 .

After we prescribed $\xi$ and $\mu$ and got $\phi_{\xi, \mu}$, the coefficients of 1st mode and high mode change accordingly, while from Theorem 4.1, the first mode and high mode are still solvable and the solutions enjoy similar estimates as in Subsection 3.2 and Theorem 3.5. We denote the corresponding solutions as $\tilde{w}_{\xi, \mu}$ and $\eta_{\xi, \mu}$.

We have

$$
\begin{aligned}
\Pi_{0}\left(H\left(\mathcal{D}_{\phi_{\xi, \mu}, p_{0}, \varepsilon}\left(\tilde{w}_{\xi, \mu}, \eta_{\xi, \mu}\right)\right)-\frac{2}{\varepsilon}\right)= & \Pi_{0}\left(\left(\varepsilon^{2}+\varepsilon \rho\right) E_{\xi, \mu}+\left(\varepsilon+\varepsilon^{-1} \rho\right) L_{\xi, \mu}\left(\tilde{w}_{\xi, \mu}, \eta_{\xi, \mu}\right)\right. \\
& \left.+\varepsilon^{-1}(1+\rho) Q_{\xi, \mu}\left(\tilde{w}_{\xi, \mu}, \eta_{\xi, \mu}\right)\right)+\varepsilon^{2} \mu \\
& +\Pi_{0}\left(F_{3}\left(\phi_{\xi, \mu}, \zeta_{\xi, \mu}\right) \star R_{3}\left(\eta_{\xi, \mu}\right)\right) \\
& +F_{4}\left(\phi_{\xi, \mu}, \zeta_{\xi, \mu}\right) \star \xi+\varepsilon^{2} \omega \phi_{\xi, \mu}^{-1} \frac{\partial \phi_{\xi, \mu}}{\partial \psi}
\end{aligned}
$$


where $\zeta_{\xi, \mu}=\partial_{\psi} \phi_{\xi, \mu}$.

Note that $\Pi_{0}\left(F_{3}\left(\phi_{\xi, \mu}, \zeta_{\xi, \mu}\right) \star R_{3}\left(\eta_{\xi, \mu}\right)\right)=F_{4}\left(\phi_{\xi, \mu}, \zeta_{\xi, \mu}\right) \star \Pi_{0}\left(R_{3}\left(\eta_{\xi, \mu}\right)\right)$, because

$$
\Pi_{0}\left(R\left(\Upsilon, X_{0}, \eta_{\xi, \mu}, \Upsilon\right)-R\left(\Upsilon_{\theta}, X_{0}, \eta_{\xi, \mu}, \Upsilon_{\theta}\right)\right)=0 .
$$

We want to choose $\xi$ and $\mu$ such that

$$
\left\{\begin{array}{l}
\left(\varepsilon^{2}+\varepsilon \rho\right) E_{\xi, \mu}+\left(\varepsilon+\varepsilon^{-1} \rho\right) L_{\xi, \mu}\left(\tilde{w}_{\xi, \mu}, \eta_{\xi, \mu}\right) \quad+\varepsilon^{-1}(1+\rho) Q_{\xi, \mu}\left(\tilde{w}_{\xi, \mu}, \eta_{\xi, \mu}\right)+\varepsilon^{2} \mu=0, \\
\Pi_{0}\left(R_{3}\left(\eta_{\xi, \mu}\right)\right)+\xi=0 .
\end{array}\right.
$$

From Definition 4, Lemma 4.2, 4.3, we know a fixed point argument works and we will find the solution $(\hat{\xi}, \hat{\mu})$ such that

$$
H\left(\mathcal{D}_{\phi_{\hat{\xi}, \hat{\mu}}, p_{0}, \varepsilon}\left(\tilde{w}_{\hat{\xi}, \hat{\mu}}, \eta_{\hat{\xi}, \hat{\mu}}\right)\right)=\frac{2}{\varepsilon}+\varepsilon^{2} \omega_{\hat{\xi}, \hat{\mu}} \phi_{\hat{\xi}, \hat{\mu}}^{-1} \frac{\partial \phi_{\hat{\xi}, \hat{\mu}}}{\partial \psi} .
$$

In Subsection 4.2 we remove the term $\varepsilon^{2} \omega_{\hat{\xi}, \hat{\mu}} \phi_{\hat{\xi}, \hat{\mu}}^{-1} \frac{\partial \phi_{\hat{\xi}, \hat{\mu}}}{\partial \psi}$, by considering the energy of the surface. Letting $p_{0}$ vary on the geodesic, we find the critical points of the energy functional, which correspond to the case $\omega_{\hat{\xi}, \hat{\mu}}=0$. So we can overcome the first difficulty.

The third difficulty exists even when we consider nonlinear ODE in 0th mode. Generally $\eta=O\left(\varepsilon^{2}\right)$ is too big to solve the whole system.

The key to control the size of $\phi_{\xi, \mu}$ is the Delaunay parameter function $\tau=$ $-\phi^{2}+\frac{\phi}{\sqrt{1+\phi_{\psi}^{2}}}$. For the solution $\phi_{\xi, \mu}$ of 27 ,,$F_{4} \xi$ influence the size of $\phi_{\xi, \mu}$ through the following equation

$$
\frac{d \tau}{d \psi}=\phi \phi_{\psi} \rho
$$

Here $\xi$ represents $R_{4}(\eta)$ whose norm is $C_{x_{0}}^{1}$. For $F_{4}$, we have

Lemma 3.6. ("average 0" lemma) Suppose $\phi_{\tau_{0}}$ is the solution to (2) and $\psi \in$ $\left[a_{\tau_{0}}, b_{\tau_{0}}\right]$ is one period of $\phi_{\tau_{0}}(\psi)$. Then we have

$$
\int_{a_{\tau_{0}}}^{b_{\tau_{0}}} \phi_{\tau_{0}}\left(\frac{\partial \phi_{\tau_{0}}}{\partial \psi}\right) F_{4}\left(\phi_{\tau_{0}}, \frac{\partial \phi_{\tau_{0}}}{\partial \psi}\right) d \psi=0
$$

We prove this lemma in Appendix B

Using this lemma, we can overcome the third difficulty. Indeed, if in the $C_{x_{0}}^{1}$ norm, $\eta$ has a variation of size $O\left(\varepsilon^{2}\right)$, because of Lemma 3.6, its influence on 0th mode is only as if it were $O\left(\varepsilon^{3}\right)$. The function $\phi_{\tau_{0}}\left(\frac{\partial \phi_{\tau_{0}}}{\partial \psi}\right) F_{4}\left(\phi_{\tau_{0}}, \frac{\partial \phi_{\tau_{0}}}{\partial \psi}\right)$ is in no sense an "odd" function. So it is nontrivial that such a cancellation result holds.

\subsection{0th mode}

\subsubsection{The main theorem of 0th mode}

Theorem 3.7. For fixed $\tau_{0} \in\left(0, \frac{1}{4}\right), C_{1}, C_{2}>0$, we can choose $C_{3}, C_{4}>0$ and $\delta_{0}>0$, such that when $\varepsilon \leq \delta_{0}$ and $\varepsilon \in \operatorname{PS}\left(L_{\Gamma}, \tau_{0}\right)$, for every $\|\xi\|_{C_{x_{0}}^{1}} \leq$ 
$C_{1} \varepsilon^{2},\|\mu\|_{C_{\varepsilon}^{\alpha}} \leq C_{2}$, we can find unique

$$
\left|\omega_{\xi, \mu}\right| \leq C_{3},\left|\phi(0)_{\xi, \mu}-\frac{1-\sqrt{1-4 \tau_{0}}}{2}\right| \leq C_{4} \varepsilon^{2}
$$

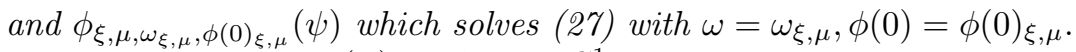

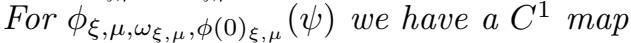

$$
\tilde{\Phi}=\tilde{\Phi}_{\xi, \mu, \tau_{0}}: \Gamma \rightarrow \Gamma
$$

such that

$$
\begin{aligned}
& \left|\phi_{\xi, \mu, \omega_{\xi, \mu}, \phi(0)_{\xi, \mu}}(\psi)-\phi_{\tau_{0}}(\tilde{\Phi}(\psi))\right|+\left|\frac{\partial \phi_{\xi, \mu, \omega_{\xi, \mu}, \phi(0)_{\xi, \mu}}}{\partial \psi}(\psi)-\frac{\partial \phi_{\tau_{0}}}{\partial \tilde{\Phi}(\psi)}(\tilde{\Phi}(\psi))\right| \\
\leq & C\left(\tau_{0}, C_{1}, C_{2}\right) \varepsilon^{2}
\end{aligned}
$$

and

$$
\left\{\begin{array}{l}
|\tilde{\Phi}(\psi)-\psi| \leq C\left(\tau_{0}, C_{1}, C_{2}\right) \varepsilon, \\
\left|\tilde{\Phi}^{\prime}(\psi)-1\right| \leq C\left(\tau_{0}, C_{1}, C_{2}\right) \varepsilon^{2} .
\end{array}\right.
$$

Moreover suppose $\psi_{i}$ is the ith local minimum point of $\phi_{\xi, \mu, \omega_{\xi, \mu}, \phi(0)_{\xi, \mu}}$, then $\tilde{\Phi}\left(\psi_{i}\right)$ is the ith local minimum point of $\phi_{\tau_{0}}$. In particular $\tilde{\Phi}(0)=0, \tilde{\Phi}\left(L_{\Gamma}\right)=L_{\Gamma}$.

Moreover,

$$
\begin{aligned}
\left|\omega_{\xi_{2}, \mu_{2}}-\omega_{\xi_{1}, \mu_{1}}\right| & \leq C\left(\tau_{0}, C_{1}, C_{2}\right)\left(\left\|\mu_{2}-\mu_{1}\right\|_{C_{\varepsilon}^{\alpha}}+\frac{1}{\varepsilon}\left\|\xi_{2}-\xi_{1}\right\|_{C_{x_{0}}^{1}}\right), \\
\left|\phi(0)_{\xi_{2}, \mu_{2}}-\phi(0)_{\xi_{1}, \mu_{1}}\right| & \leq C\left(\tau_{0}, C_{1}, C_{2}\right)\left(\varepsilon^{2}\left\|\mu_{2}-\mu_{1}\right\|_{C_{\varepsilon}^{\alpha}}+\varepsilon\left\|\xi_{2}-\xi_{1}\right\|_{C_{x_{0}}^{1}}^{1}\right),
\end{aligned}
$$

and

$$
\begin{aligned}
& \left\|\phi_{\xi_{2}, \mu_{2}, \omega_{\xi_{2}, \mu_{2}}, \tau(0)_{\xi_{2}, \mu_{2}}}(\psi)-\phi_{\xi_{1}, \mu_{1}, \omega_{\xi_{1}, \mu_{1}}, \tau(0)_{\xi_{1}, \mu_{1}}}(\psi)\right\|_{C_{\varepsilon}^{2, \alpha}} \\
& \leq C\left(\tau_{0}, C_{1}, C_{2}\right)\left(\varepsilon\left\|\mu_{2}-\mu_{1}\right\|_{C_{\varepsilon}^{\alpha}}+\left\|\xi_{2}-\xi_{1}\right\|_{C_{x_{0}}^{1}}\right) .
\end{aligned}
$$

The proof of Theorem 3.7 is the most technical part of this paper. We prove it in six steps.

From Step 1 to Step 4, we will study the following initial value problem instead of (27).

$$
\begin{cases}\phi_{\psi \psi} & -\phi^{-1}\left(1+\phi_{\psi}^{2}\right)+(2+\rho)\left(1+\phi_{\psi}^{2}\right)^{\frac{3}{2}}=0 \\ \phi_{\psi}(0)=0 \\ \phi(0) \quad=\frac{1-\sqrt{1-4 \tau(0)}}{2}\end{cases}
$$

where $\rho$ is defined in (27). In Step 1, we prove the local existence of (30); In Step 2 , we prove the existence of the solution of $\left[30\right.$ on the whole interval $\left[0, \frac{L_{\Gamma}}{\varepsilon}\right]$. In Step 3, we do some basic estimates for the solution. In Step 4, we analyze the linearized equations of (30), which will be used in the next two steps. In Step 5 , we adjust $\omega$ and $\tau(0)$ to match the boundary data on both sides of $\psi=0$ (or $\psi=\frac{L_{\Gamma}}{\varepsilon}$ ) and get the global solution to 27 . In Step 6 , we derive the estimates in Theorem 3.7 . 
Step 1. Local existence and uniqueness of ODE (27) Fix $C_{1}, C_{2}, C_{3}$ and $C\left(\tau_{0}\right)$ in (28). Choose $A_{1}, A_{2}, B_{1}, B_{2}, K_{1}, K_{2}$ that only depend on $\tau_{0}$ such that

$$
0<A_{1}<A_{2}<\frac{1-\sqrt{1-4 \tau(0)}}{2}<\frac{1+\sqrt{1-4 \tau(0)}}{2}<B_{2}<B_{1}<1,
$$

and

$$
K_{1}-1=K_{2}=1+\sup \left\{\left|\phi_{\psi}\right| ;-\phi^{2}+\frac{\phi}{\sqrt{1+\phi_{\psi}}}=\tau_{0}-C\left(\tau_{0}\right)\right\} .
$$

Define $C_{A_{i}, B_{i}, K_{i}}^{1}([0, T])=\left\{\phi(\psi) \in C^{1}([0, T]) ; A_{i} \leq \phi(\psi) \leq B_{i},\left|\phi_{\psi}\right| \leq K_{i}\right\}, i=$ 1,2 . We have

Lemma 3.8. If $\phi(\psi) \in C_{A_{2}, B_{2}, K_{2}}^{1}([0, T])$ solves (30) for $T \geq 0$. Then for some $\delta>0$ which only depends on $\tau_{0}$, this solution can be uniquely extended to $\phi(\psi) \in$ $C_{A_{1}, B_{1}, K_{1}}^{1}([0, T+\delta])$.

Proof. Denote $\frac{\partial \phi}{\partial \psi}$ by $\zeta$. Then $(\phi, \zeta)$ satisfies the following system

$$
\begin{cases}\phi_{\psi}=\zeta, & \phi(0)=\frac{1-\sqrt{1-4 \tau(0)}}{2}, \\ \zeta_{\psi}=\phi^{-1}\left(1+\zeta^{2}\right)-(2+\rho)\left(1+\zeta^{2}\right)^{\frac{3}{2}}, & \zeta(0)=0\end{cases}
$$

The right hand side is uniformly bounded in $(\phi, \zeta, \psi)$ and is a Lipschitz function with respect to $(\phi, \zeta)$ in the domain

$$
\left\{\begin{array}{l}
|\zeta|<K_{1} \\
A_{1}<\phi<B_{1} \\
0<\psi<\frac{L_{\Gamma}}{\varepsilon}
\end{array}\right.
$$

The $C^{0}$ norm and Lipschitz constant of the right hand side only depend on $\tau_{0}$. So from standard theory of ODE we get the conclusion.

Step 2. Existence on $\left[0, \frac{L_{\Gamma}}{\varepsilon}\right]$ To get the existence of the solution on $\left[0, \frac{L_{\Gamma}}{\varepsilon}\right]$, we need to do apriori estimates for the solution. The key to do this is the first integral

$$
\tau\left(\phi, \phi_{\psi}\right)=-\phi^{2}+\frac{\phi}{\sqrt{1+\phi_{\psi}^{2}}} .
$$

The following graph is the phase space of 27$)$. We choose $\tau_{0} \in\left(0, \frac{1}{4}\right)$ and $\delta_{1}$ small. We denote the closure of the domain between the outside and inside circle by $A\left(\tau_{0}, \delta_{1}\right)$. And we denote $\left\{\phi(\psi) \in C^{1}\left(\left[0, T_{0}\right]\right) \mid\left(\phi, \phi_{\psi}\right) \in A\left(\tau_{0}, \delta_{1}\right)\right\}$ as $C_{A\left(\tau_{0}, \delta_{1}\right)}^{1}\left(\left[0, T_{0}\right]\right)$. 


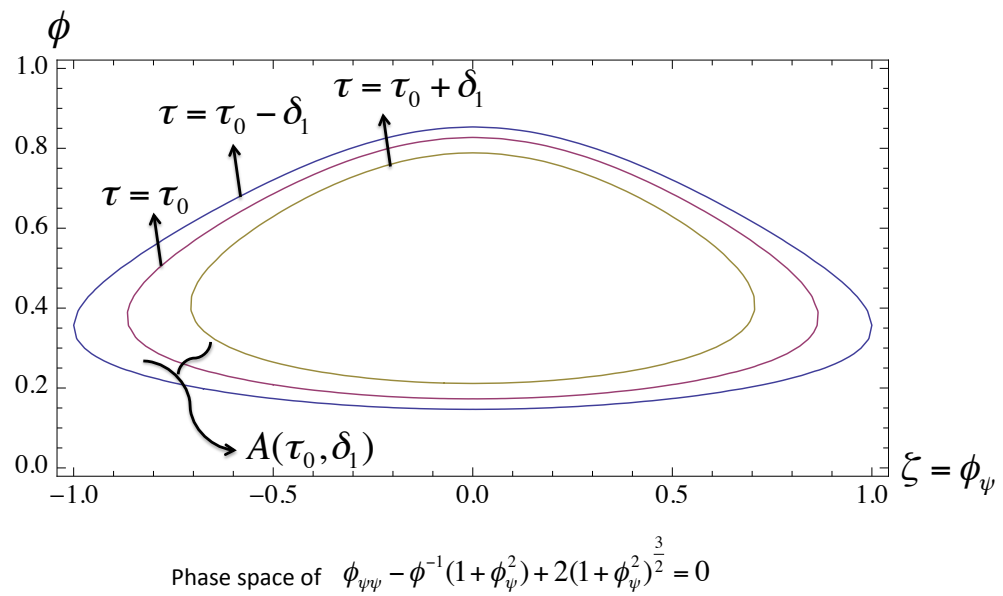

We have,

Lemma 3.9. (Existence on $\left[0, \frac{L_{\Gamma}}{\varepsilon}\right]$ ) For fixed $\xi, \mu, \omega, \tau(0)$ which satisfy 208), there is $\tilde{\delta}>0$ such that when $\tilde{\delta}>\varepsilon \in \operatorname{PS}\left(L_{\Gamma}, \tau_{0}\right)$, there is a unique solution $\phi_{\xi, \mu, \omega, \tau(0)}(\psi) \in C_{A\left(\tau_{0}, \delta_{1}\right)}^{1}\left(\left[0, \frac{1}{\varepsilon} L_{\Gamma}\right]\right)$ to 30 ).

Proof. First one can choose $\tilde{\delta}$ small that $\left|\tau(0)-\tau_{0}\right| \leq C\left(\tau_{0}\right) \tilde{\delta}<\delta_{1}$. Suppose for contradiction the lemma were false. From Step 1, we assume $T<\frac{L_{\Gamma}}{\varepsilon}$ is the maximal value that the solution $\phi(\psi)$ can be extended in $C_{A\left(\tau_{0}, \delta_{1}\right)}^{1}([0, T])$. So we must have $(\phi(T), \zeta(T)) \in \partial A\left(\tau_{0}, \delta_{1}\right)$, that is to say $\tau(\phi(T), \zeta(T))=\tau_{0} \pm \delta_{1}$.

From easy calculations,

$$
\frac{d \tau}{d \psi}=\rho(\psi) \phi \phi_{\psi},
$$

where $\|\rho(\psi)\|_{C^{0}} \leq C\left(\varepsilon^{2}+\varepsilon\|\xi\|_{C^{0}}+\varepsilon^{3}\|\mu\|_{C^{0}}+\varepsilon^{3}|\omega|\right)$. So

$$
\begin{aligned}
& |\tau(\phi(T), \zeta(T))-\tau(\phi(0), 0)| \\
= & \left|\int_{0}^{T} \rho(\psi) \phi \phi_{\psi} d \psi\right| \\
\leq & C T\left(\varepsilon^{2}+\varepsilon\|\xi\|_{C^{0}}+\varepsilon^{3}\|\mu\|_{C^{0}}+\varepsilon^{3}|\omega|\right) \\
\leq & C\left(\varepsilon+\|\xi\|_{C^{0}}+\varepsilon^{2}\|\mu\|_{C^{0}}+\varepsilon^{2}|\omega|\right) \\
\leq & C\left(\varepsilon+\left(C_{1}+C_{2}+C_{3}\right) \varepsilon^{2}\right) .
\end{aligned}
$$

So we have

$$
\begin{aligned}
& \left|\tau(\phi(T), \zeta(T))-\tau_{0}\right| \\
\leq & |\tau(\phi(T), \zeta(T))-\tau(\phi(0), 0)|+\left|\tau(\phi(0), 0)-\tau_{0}\right| \\
\leq & C\left(\varepsilon+\left(C_{1}+C_{2}+C_{3}\right) \varepsilon^{2}\right)+C\left(\tau_{0}\right) \varepsilon .
\end{aligned}
$$

So we can choose $\tilde{\delta}$ small enough, such that, when $\varepsilon \leq \tilde{\delta}$

$$
\left|\tau(\phi(T), \zeta(T))-\tau_{0}\right| \leq \frac{\delta_{1}}{2}
$$


which makes $\tau(\phi(T), \zeta(T))=\tau_{0} \pm \delta_{1}$ impossible. So when $\varepsilon \leq \tilde{\delta}$, for any prescription of $\xi, \mu, \omega, \tau(0)$ which satisfies $(28)$, the solution $\phi_{\xi, \mu, \omega, \tau(0)}(\psi)$ exists for $\psi \in\left[0, \frac{L_{\Gamma}}{\varepsilon}\right]$.

Step 3. Estimates of $\phi_{\xi, \mu, \omega, \tau(0)}(\psi) \quad$ For simplicity we denote $\left(\phi_{\xi, \mu, \omega, \tau(0)}(\psi), \frac{\partial \phi_{\xi, \mu, \omega, \tau(0)}(\psi)}{\partial \psi}\right)$ as $(\phi(\psi), \zeta(\psi))$. From (31) we know, $\tau(\phi(\psi), \zeta(\psi))$ will keeps in $C\left(C_{1}, C_{2}, C_{3}, \tau_{0}\right) \varepsilon$ neighborhood of $\tau(0)=\tau(\phi(0), 0)$. Actually we can improve this estimate to $C \varepsilon^{2}$ neighborhood. This result comes from a simple observation. Note that

$$
\begin{aligned}
& |\tau(\phi(T), \zeta(T))-\tau(\phi(0), 0)| \\
= & \left|\int_{0}^{T} \rho(\psi) \phi \phi_{\psi} d \psi\right| \\
= & \mid \int_{0}^{T} \phi \phi_{\psi}\left(-\varepsilon^{2} F_{1}\left(\phi, \phi_{\psi}\right) \star \Pi_{0}\left(R_{1}\right)+\varepsilon F_{4}\left(\phi, \phi_{\psi}\right) \xi\right. \\
& \left.+\varepsilon^{3} \mu+\varepsilon^{3} \omega \phi^{-1} \phi_{\psi}\right) d \psi \mid .
\end{aligned}
$$

First $\varepsilon \xi=O\left(\varepsilon^{3}\right), \varepsilon^{3} \mu=O\left(\varepsilon^{3}\right), \varepsilon^{3} \omega \phi^{-1} \phi_{\psi}=O\left(\varepsilon^{3}\right)$. The integral of these three terms is $O\left(\varepsilon^{2}\right)$ because $0 \leq T \leq \frac{L_{\Gamma}}{\varepsilon}$. At first glance the integral of $\phi \frac{\partial \phi}{\partial \psi}\left(-\varepsilon^{2}\right) F_{1}\left(\phi, \phi_{\psi}\right) \star$ $\Pi_{0}\left(R_{1}\right)$ is of order $O(\varepsilon)$. However there is a cancellation property. Note that $F_{1}\left(\phi, \phi_{\psi}\right) \phi \frac{\partial \phi}{\partial \psi}$ nearly has 0 average in one period (up to an error of order $O(\varepsilon)$ ). Also we know $\nabla_{\psi} R\left(\Upsilon, \Upsilon_{\theta}, \Upsilon, \Upsilon_{\theta}\right)=O(\varepsilon)$. So in one period the integral is only of size $\varepsilon^{3}$. When $T$ is as large as $\frac{L_{\Gamma}}{\varepsilon}$, the integral is of size $\varepsilon^{2}$.

To be precise, we have

Lemma 3.10. There exists $C=C\left(C_{1}, C_{2}, C_{3}, \tau_{0}\right)$ which doesn't depend on $\varepsilon$ such that for $\psi \in\left[0, \frac{L_{\Gamma}}{\varepsilon}\right],(\phi(\psi), \zeta(\psi)) \in A\left(\tau(0), C \varepsilon^{2}\right)$.

Proof. We know that for each $\psi \in\left[0, \frac{L_{\Gamma}}{\varepsilon}\right],|\tau(\phi(\psi), \zeta(\psi))-\tau(\phi(0), 0)| \leq C(\varepsilon+$ $\left.\|\xi\|_{C^{0}}+\varepsilon^{2}\|\mu\|_{C^{0}}+\varepsilon^{2}|\omega|\right)$ from (31). Suppose $\phi_{\tau(0)}(\psi)$ defines the standard Delaunay surface with parameter $\tau \equiv \tau(\phi(0), 0)$ and $\zeta_{\tau(0)}(\psi)=\frac{d}{d \psi} \phi_{\tau(0)}(\psi)$. We assume the arc length parameter of the curve $\left(\phi_{\tau(0)}(\psi), \zeta_{\tau(0)}(\psi)\right)$ in the phase space is $s_{0}$ $\left(s_{0}\right.$ is a multi-valued function on the curve $\left.\left(\phi_{\tau(0)}, \zeta_{\tau(0)}\right)\right)$. We can extend $s_{0}$ to a neighborhood of $\left(\phi_{\tau(0)}(\psi), \zeta_{\tau(0)}(\psi)\right)$ such that $\frac{\partial}{\partial \tau} \perp \frac{\partial}{\partial s_{0}}$ holds everywhere in this neighborhood. We know that in this neighborhood

$$
d \phi^{2}+d \zeta^{2}=<\partial_{s_{0}}, \partial_{s_{0}}>d s_{0}^{2}+<\partial_{\tau}, \partial_{\tau}>d \tau^{2}
$$

where $<,>$ denotes the inner product of the metric $d \phi^{2}+d \zeta^{2}$. We know that $<\partial_{s_{0}}, \partial_{s_{0}}>=1$ on $\left(\phi_{\tau(0)}(\psi), \zeta_{\tau(0)}(\psi)\right)$. Regard $\left(s_{0}, \tau\right)$ as new local coordinate of $A(\tau(0), C \varepsilon)$. We define a continuous map $\Phi=\Phi_{\xi, \mu, \omega, \tau(0)}:\left[0, \frac{L_{\Gamma}}{\varepsilon}\right] \rightarrow \mathbb{R}$ such that

$$
s_{0}(\phi(\psi), \zeta(\psi))=s_{0}\left(\phi_{\tau(0)}(\Phi(\psi)), \zeta_{\tau(0)}(\Phi(\psi))\right) .
$$

Here is a graph which illustrates the definition of $\Phi$. 


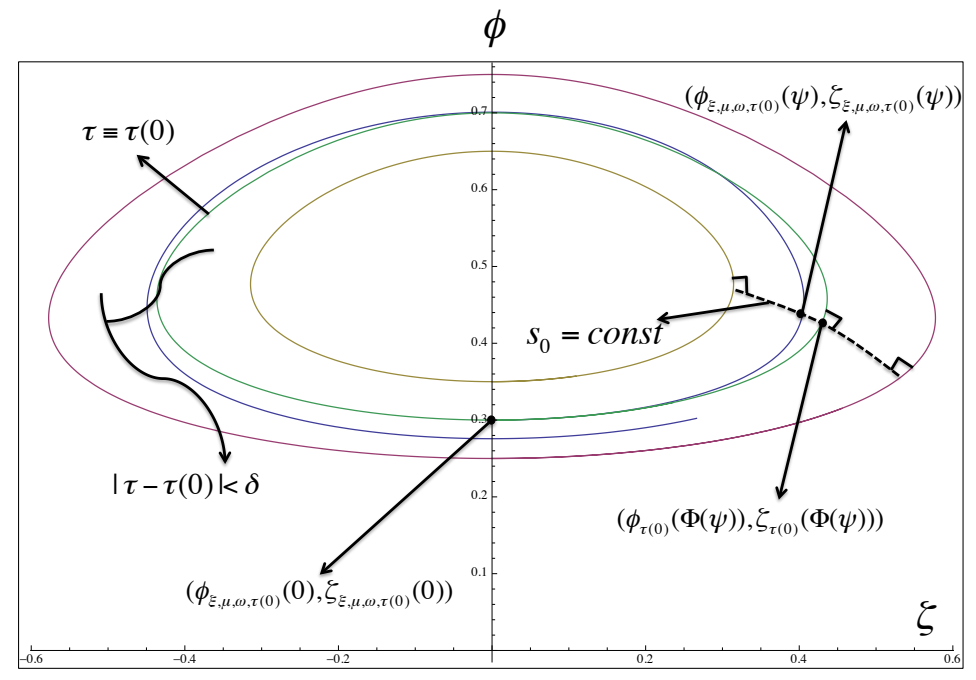

The definition of $\Phi(\psi)$

Remark. From (34) we see, if $\zeta(\psi)=0$, then $\zeta_{\tau(0)}(\Phi(\psi))=0$. If $\psi$ is a local minimum point of $\phi, \Phi(\psi)$ is a local minimum point of $\phi_{\tau(0)}$.

So we have

$$
\begin{cases}\left|\phi(\psi)-\phi_{\tau(0)}(\Phi(\psi))\right| & \leq C\left(\tau_{0}\right)\left(\varepsilon+\left(C_{1}+C_{2}+C_{3}\right) \varepsilon^{2}\right) \\ \left|\zeta(\psi)-\zeta_{\tau(0)}(\Phi(\psi))\right| & \leq C\left(\tau_{0}\right)\left(\varepsilon+\left(C_{1}+C_{2}+C_{3}\right) \varepsilon^{2}\right) .\end{cases}
$$

Lemma 3.11.

$$
\left\{\begin{array}{l}
\Phi(0)=0 \\
|\Phi(\psi)-\psi| \leq \frac{C\left(\tau_{0}\right)}{\varepsilon}\left(\varepsilon+\left(C_{1}+C_{2}+C_{3}\right) \varepsilon^{2}\right) \\
\left|\Phi^{\prime}(\psi)-1\right| \leq C\left(\tau_{0}\right)\left(\varepsilon+\left(C_{1}+C_{2}+C_{3}\right) \varepsilon^{2}\right) .
\end{array}\right.
$$

In particular, $\Phi:\left[0, \frac{L_{\Gamma}}{\varepsilon}\right] \rightarrow \Phi\left(\left[0, \frac{L_{\Gamma}}{\varepsilon}\right]\right)$ is invertible.

We prove Lemma 3.11 in Appendix C.

Now we continue to prove Lemma 3.9. Let $\left[\tilde{\psi}_{i}, \tilde{\psi}_{i+1}\right]$ be the $i$ th period of $\phi_{\tau(0)}(\psi)$. We assume $\psi_{i}=\Phi^{-1}\left(\tilde{\psi}_{i}\right)$. We know $\phi_{\psi}\left(\psi_{i}\right)=0$. From 31 35, we have 


$$
\begin{aligned}
& \left|\int_{\psi_{i}}^{\psi_{i+1}} \phi \phi_{\psi} F_{1}\left(\phi, \phi_{\psi}\right) \star \Pi_{0}\left(R_{1}\right) d \psi\right| \\
\leq & \left.\left|\int_{\tilde{\psi}_{i}}^{\tilde{\psi}_{i+1}} \phi_{\tau(0)} \frac{\partial \phi_{\tau(0)}}{\partial \psi} F_{1}\left(\phi_{\tau(0)}, \frac{\partial \phi_{\tau(0)}}{\partial \psi}\right)\right|_{\varsigma} \star \Pi_{0}\left(R_{1}\right)\right|_{\Phi^{-1}(\varsigma)} d \varsigma \mid \\
& +C\left(\tau_{0}\right)\left(\varepsilon+\|\xi\|_{C^{0}}+\varepsilon^{2}\|\mu\|_{C^{0}}+\varepsilon^{2}|\omega|\right) \\
= & \left|\int_{\tilde{\psi}_{i}}^{\tilde{\psi}_{i+1}} \tilde{\chi}(\varsigma) \star \frac{\partial}{\partial \varsigma} \Pi_{0}\left(R_{1}\right)\right|_{\Phi^{-1}(\varsigma)} d \varsigma \mid \\
& +C\left(\tau_{0}\right)\left(\varepsilon+\|\xi\|_{C^{0}}+\varepsilon^{2}\|\mu\|_{C^{0}}+\varepsilon^{2}|\omega|\right) \\
= & C\left(\tau_{0}\right)\left(\varepsilon+\left(C_{1}+C_{2}+C_{3}\right) \varepsilon^{2}\right),
\end{aligned}
$$

where $\tilde{\chi}(\varsigma)$ is the primitive of $\phi_{\tau(0)} \frac{\partial \phi_{\tau(0)}}{\partial \psi} F_{1}\left(\phi_{\tau(0)}, \frac{\partial \phi_{\tau(0)}}{\partial \psi}\right)$ and $|\tilde{\chi}(s)|_{C^{0}} \leq C\left(\tau_{0}\right) \varepsilon$. Now by dividing $\left[0, \frac{L_{\Gamma}}{\varepsilon}\right]$ into "periods" of $\phi(\psi)$, it is easy to prove that, for any $\bar{\psi} \in\left[0, \frac{L_{\Gamma}}{\varepsilon}\right]$

$$
\begin{aligned}
& \left|\int_{0}^{\bar{\psi}} \phi \phi_{\psi} \varepsilon^{2} F_{1}\left(\phi, \phi_{\psi}\right) \star \Pi_{0}\left(R_{1}\right) d \psi\right| \\
\leq & C\left(\tau_{0}\right) \varepsilon\left(\varepsilon+\left(C_{1}+C_{2}+C_{3}\right) \varepsilon^{2}\right) .
\end{aligned}
$$

So we can get better estimate for $\tau$,

$$
|\tau(\phi(T), \zeta(T))-\tau(\phi(0), 0)| \leq C\left(\tau_{0}\right)\left(1+C_{1}+C_{2}+C_{3}\right) \varepsilon^{2}
$$

for $T \in\left[0, \frac{1}{\varepsilon} L_{\Gamma}\right]$.

Now we can get better estimates for $\Phi$ and $\phi(\psi)$, i.e.

$$
\left\{\begin{array}{l}
\left|\phi(\psi)-\phi_{\tau(0)}(\Phi(\psi))\right| \leq C\left(\tau_{0}\right)\left(\varepsilon^{2}+\left(C_{1}+C_{2}+C_{3}\right) \varepsilon^{2}\right) \\
\left|\zeta(\psi)-\zeta_{\tau(0)}(\Phi(\psi))\right| \leq C\left(\tau_{0}\right)\left(\varepsilon^{2}+\left(C_{1}+C_{2}+C_{3}\right) \varepsilon^{2}\right) .
\end{array}\right.
$$

and

$$
\left\{\begin{array}{l}
\Phi(0)=0, \\
|\Phi(\psi)-\psi| \leq \frac{C\left(\tau_{0}\right)}{\varepsilon}\left(\varepsilon^{2}+\|\xi\|_{C^{0}}+\varepsilon^{2}\|\mu\|_{C^{0}}+\varepsilon^{2}|\omega|\right), \\
\left|\Phi^{\prime}(\psi)-1\right| \leq C\left(\tau_{0}\right)\left(\varepsilon^{2}+\|\xi\|_{C^{0}}+\varepsilon^{2}\|\mu\|_{C^{0}}+\varepsilon^{2}|\omega|\right) .
\end{array}\right.
$$

\section{Corollary 3.12.}

$$
\begin{aligned}
& \left\|\phi(\psi)-\phi_{\tau(0)}(\psi)\right\|_{C_{\varepsilon}^{2, \alpha}} \\
\leq & C\left(\tau_{0}\right) \frac{1}{\varepsilon}\left(\varepsilon^{2}+\|\xi\|_{C_{x_{0}}^{1}}+\varepsilon^{2}\|\mu\|_{C_{\varepsilon}^{\alpha}}+\varepsilon^{2}|\omega|\right) \\
\leq & C\left(\tau_{0}\right)\left(1+C_{1}+C_{2}+C_{3}\right) \varepsilon .
\end{aligned}
$$


Proof. From 38, 39 and $\zeta_{\tau(0)}, \frac{\partial^{2} \phi_{\tau(0)}}{\partial \psi^{2}}$ are uniformly bounded by $C\left(\tau_{0}\right)$, we have

$$
\begin{aligned}
\left|\phi(\psi)-\phi_{\tau(0)}(\psi)\right| \leq & \left|\phi(\psi)-\phi_{\tau(0)}(\Phi(\psi))\right|+\left|\phi_{\tau(0)}(\Phi(\psi))-\phi_{\tau(0)}(\psi)\right| \\
\leq & C\left(\tau_{0}\right)\left(\varepsilon^{2}+\|\xi\|_{C^{0}}+\varepsilon^{2}\|\mu\|_{C^{0}}+\varepsilon^{2}|\omega|\right) \\
& +\sup \left|\zeta_{\tau(0)}\right| \cdot|\Phi(\psi)-\psi| \\
\leq & \frac{C\left(\tau_{0}\right)}{\varepsilon}\left(\varepsilon^{2}+\|\xi\|_{C^{0}}+\varepsilon^{2}\|\mu\|_{C^{0}}+\varepsilon^{2}|\omega|\right)
\end{aligned}
$$

and in the same way

$$
\left|\zeta(\psi)-\zeta_{\tau(0)}(\psi)\right| \leq \frac{C\left(\tau_{0}\right)}{\varepsilon}\left(\varepsilon^{2}+\|\xi\|_{C^{0}}+\varepsilon^{2}\|\mu\|_{C^{0}}+\varepsilon^{2}|\omega|\right) .
$$

$\left|\phi^{-1}-\phi_{\tau(0)}^{-1}\right|$ also has similar estimates as $\phi$ and $\phi_{\tau(0)}$ has positive lower bounds. Finally from (27), the definition of $\phi_{\tau(0)}$ and $(38) \sqrt{41)}$ we have

$$
\left\|\frac{\partial^{2} \phi}{\partial \psi^{2}}(\psi)-\frac{\partial^{2} \phi_{\tau(0)}}{\partial \psi^{2}}(\psi)\right\|_{C_{\varepsilon}^{\alpha}} \leq \frac{C\left(\tau_{0}\right)}{\varepsilon}\left(\varepsilon^{2}+\|\xi\|_{C_{x_{0}}^{1}}+\varepsilon^{2}\|\mu\|_{C_{\varepsilon}^{\alpha}}+\varepsilon^{2}|\omega|\right) .
$$

Corollary 3.13. Suppose $F\left(\theta_{1}, \theta_{2}\right)$ is a function with the following property. For any $\tilde{C}_{1}\left(\tau_{0}\right), \tilde{C}_{2}\left(\tau_{0}\right)$, there exists $\widetilde{C}_{3}\left(\tilde{C}_{1}, \tilde{C}_{2}\right)$ such that

$$
\sup _{\left|\theta_{1}\right| \leq \tilde{C}_{1},\left|\theta_{2}\right| \leq \tilde{C}_{2}}|F|+\left|\partial_{\theta_{1}} F\right|+\left|\partial_{\theta_{2}} F\right| \leq \tilde{C}_{3} .
$$

Then for any

$$
\|R(\psi)\|_{C_{x_{0}}^{1}(\Gamma)} \leq C
$$

we have

$$
\begin{aligned}
& \left|\int_{0}^{\psi} F\left(\phi, \phi_{\psi}\right) R(\psi) d \psi-\int_{0}^{\Phi(\psi)} F\left(\phi_{\tau(0)}(\varsigma), \frac{\partial \phi_{\tau(0)}}{\partial \varsigma}\right) R(\varsigma) d \varsigma\right| \\
\leq & \frac{C\left(\tau_{0}\right)}{\varepsilon}\left(\varepsilon^{2}+\|\xi\|_{C^{0}}+\varepsilon^{2}\|\mu\|_{C^{0}}+\varepsilon^{2}|\omega|\right)\|R\|_{C_{x_{0}}^{1}} .
\end{aligned}
$$

In particular, when $R(\psi) \equiv 1$,

$$
\begin{aligned}
& \left|\int_{0}^{\psi} F\left(\phi, \phi_{\psi}\right) d \psi-\int_{0}^{\Phi(\psi)} F\left(\phi_{\tau(0)}(\varsigma), \frac{\partial \phi_{\tau(0)}}{\partial \varsigma}\right) d \varsigma\right| \\
\leq & \frac{C\left(\tau_{0}\right)}{\varepsilon}\left(\varepsilon^{2}+\|\xi\|_{C^{0}}+\varepsilon^{2}\|\mu\|_{C^{0}}+\varepsilon^{2}|\omega|\right) .
\end{aligned}
$$


Proof.

$$
\begin{aligned}
& \left|\int_{0}^{\psi} F\left(\phi, \phi_{\psi}\right) R(\psi) d \psi-\int_{0}^{\Phi(\psi)} F\left(\phi_{\tau(0)}(\varsigma), \frac{\partial \phi_{\tau(0)}}{\partial \varsigma}\right) R(\varsigma) d \varsigma\right| \\
\leq & \int_{0}^{\Phi(\psi)}\left|F\left(\phi, \phi_{\psi}\right)\left(\Phi^{-1}(\varsigma)\right)\left(\Phi^{-1}\right)^{\prime} R\left(\Phi^{-1}(\varsigma)\right)-F\left(\phi_{\tau(0)}(\varsigma), \frac{\partial \phi_{\tau(0)}}{\partial \varsigma}\right) R(\varsigma)\right| d \varsigma \\
\leq & \frac{C\left(\tau_{0}\right)}{\varepsilon}\left(\left|\phi\left(\Phi^{-1}(\varsigma)\right)-\phi_{\tau(0)}(\varsigma)\right||R|+\left|\phi_{\psi}\left(\Phi^{-1}(\varsigma)\right)-\frac{\partial \phi_{\tau(0)}(\varsigma)}{\partial \varsigma} \| R\right|\right. \\
& \left.+\left|\left(\Phi^{-1}\right)^{\prime}-1\right||R|+\left|\partial_{\psi} R \| \Phi^{-1}(\varsigma)-\varsigma\right|\right) \\
\leq & \frac{C\left(\tau_{0}\right)}{\varepsilon}\left(\varepsilon^{2}+\|\xi\|_{C^{0}}+\varepsilon^{2}\|\mu\|_{C^{0}}+\varepsilon^{2}|\omega|\right)\|R\|_{C_{x_{0}}^{1}} .
\end{aligned}
$$

Step 4. The linearized equations In this step we analyze the linearized equations of $(30)$. In Step 2, we have got a solution $\phi(\psi)=\phi_{\xi, \mu, \omega, \tau(0)}(\psi), \psi \in$ $\left[0, \frac{L_{\Gamma}}{\varepsilon}\right]$. We can linearize the equation in different ways. First, we fix $\xi, \mu, \omega$ and perturb the initial values. Then we fixed the initial values and perturb $\xi, \mu, \omega$.

For fixed $\xi, \mu, \omega$ we suppose $\phi_{t}$ is a class of solutions to

$$
\frac{\partial^{2} \phi}{\partial \psi^{2}}-\phi^{-1}\left(1+\left(\frac{\partial \phi}{\partial \psi}\right)^{2}\right)+(2+\rho)\left(1+\left(\frac{\partial \phi}{\partial \psi}\right)^{2}\right)^{\frac{3}{2}}=0,
$$

(with different initial values) and $\left.\frac{d}{d t} \phi_{t}\right|_{t=0}=\beta(\psi)$. Then we can calculate

$$
\begin{aligned}
& \mathcal{L}_{\xi, \mu, \omega, \tau(0)} \beta(\psi) \\
= & \frac{\partial^{2} \beta}{\partial \psi^{2}}+\left(6\left(1+\phi_{\psi}^{2}\right)^{\frac{1}{2}} \phi_{\psi}-2 \phi^{-1} \phi_{\psi}+\bar{F}_{1}\right) \frac{\partial \beta}{\partial \psi}+\left(\phi^{-2}\left(1+\phi_{\psi}^{2}\right)+\bar{F}_{2}\right) \beta \\
= & 0
\end{aligned}
$$

where $\left\|\bar{F}_{1}\right\|_{C_{\varepsilon}^{\alpha}}+\left\|\bar{F}_{2}\right\|_{C_{\varepsilon}^{\alpha}} \leq \varepsilon^{2}\left(C+C_{1}+C_{2}+C_{3}\right)$. First we analyze the fundamental solutions to the linearized equation (42). Suppose $\beta_{1}(\psi), \beta_{2}(\psi)$ satisfy

$$
\mathcal{L}_{\xi, \mu, \omega, \tau(0)} \beta_{i}(\psi)=0, \psi \in\left[0, \frac{L_{\Gamma}}{\varepsilon}\right]
$$

and

$$
\begin{gathered}
\left(\begin{array}{cc}
\beta_{1}(0) & \beta_{2}(0) \\
\frac{\partial \beta_{1}}{\partial \psi}(0) & \frac{\partial \beta_{2}}{\partial \psi}(0)
\end{array}\right)=\left(\begin{array}{cc}
1 & 0 \\
0 & 1
\end{array}\right) . \\
\text { Let } R(\psi)=\left|\begin{array}{cc}
\beta_{1}(\psi) & \beta_{2}(\psi) \\
\beta_{1}^{\prime}(\psi) & \beta_{2}^{\prime}(\psi)
\end{array}\right| . \text { Easy calculation yields } \\
\frac{d}{d \psi} \log R(\psi)=-\left(6\left(1+\phi_{\psi}^{2}\right)^{\frac{1}{2}} \phi_{\psi}-2 \phi^{-1} \phi_{\psi}+\bar{F}_{1}\right) .
\end{gathered}
$$


Suppose $\phi_{\tau(0)}$ is the solution to $(30)$, with $\rho$ replaced by 0. From Corollary 3.13 . and the fact that $6\left(1+\left(\frac{\partial \phi_{\tau(0)}}{\partial \varsigma}\right)^{2}\right)^{\frac{1}{2}} \frac{\partial \phi_{\tau(0)}}{\partial \varsigma}-2 \phi_{\tau(0)}^{-1} \frac{\partial \phi_{\tau(0)}}{\partial \varsigma}$ has 0 average in one period, we deduce that there exists $C=C\left(\tau_{0}, C_{1}, C_{2}, C_{3}\right)>0$ such that

$$
\mathrm{e}^{-C} \leq R(\psi) \leq \mathrm{e}^{C} .
$$

We define an operator

$$
\begin{aligned}
& \mathcal{L}_{\tau(0)} \beta(\varsigma) \\
= & \frac{\partial^{2} \beta}{\partial \varsigma^{2}}+\left(6\left(1+\left(\frac{\partial \phi_{\tau(0)}}{\partial \varsigma}\right)^{2}\right)^{\frac{1}{2}} \frac{\partial \phi_{\tau(0)}}{\partial \varsigma}-2 \phi_{\tau(0)}^{-1} \frac{\partial \phi_{\tau(0)}}{\partial \varsigma}\right) \frac{\partial \beta}{\partial \varsigma} \\
& +\phi_{\tau(0)}^{-2}\left(1+\left(\frac{\partial \phi_{\tau(0)}}{\partial \varsigma}\right)^{2}\right) \beta .
\end{aligned}
$$

Using Lemma 3.11 and Corollary 3.12 we can make comparison between $\mathcal{L}_{\xi, \mu, \omega, \tau(0)}$ and $\mathcal{L}_{\tau(0)}$.

We denote the $i$ th local minimum of $\phi$ in $\left[0, \frac{L_{\Gamma}}{\varepsilon}\right]$ as $\psi_{i}, i=0,1,2, \cdots$. So $\psi_{0}=0$ and $\left[\psi_{i-1}, \psi_{i}\right]$ resembles the $i$ th "period" of $\phi(\psi) . \tilde{\psi}_{i}=\Phi\left(\psi_{i}\right)$ is the $i$ th local minimum of $\phi_{\tau(0)}$. It is obvious that $\tilde{\psi}_{i}=i \tilde{\psi}_{1}$. When $\lambda \in[0,1]$, let $\psi=(1-\lambda) \psi_{i-1}+\lambda \psi_{i}$. From (38), (39) we have

$$
\begin{aligned}
& \left|\phi\left((1-\lambda) \psi_{i-1}+\lambda \psi_{i}\right)-\phi_{\tau(0)}\left((1-\lambda) \tilde{\psi}_{i-1}+\lambda \tilde{\psi}_{i}\right)\right| \\
\leq & \left|\phi(\psi)-\phi_{\tau(0)}(\Phi(\psi))\right|+\left|\phi_{\tau(0)}(\Phi(\psi))-\phi_{\tau(0)}\left((1-\lambda) \tilde{\psi}_{i-1}+\lambda \tilde{\psi}_{i}\right)\right| \\
\leq & C\left(\tau_{0}\right)\left(\varepsilon^{2}+\|\xi\|_{C^{0}}+\varepsilon^{2}\|\mu\|_{C^{0}}+\varepsilon^{2}|\omega|\right),
\end{aligned}
$$

and

$$
\begin{aligned}
& \left|\zeta\left((1-\lambda) \psi_{i-1}+\lambda \psi_{i}\right)-\zeta_{\tau(0)}\left((1-\lambda) \tilde{\psi}_{i-1}+\lambda \tilde{\psi}_{i}\right)\right| \\
\leq & C\left(\tau_{0}\right)\left(\varepsilon^{2}+\|\xi\|_{C^{0}}+\varepsilon^{2}\|\mu\|_{C^{0}}+\varepsilon^{2}|\omega|\right) .
\end{aligned}
$$

Suppose $\beta_{1, i-1}, \beta_{2, i-1}$ solves

$$
\mathcal{L}_{\xi, \mu, \omega, \tau(0)} \beta_{j, i-1}(\psi)=0, \psi \in\left[\psi_{i-1}, \psi_{i}\right], j=1,2,
$$

with

$$
\left(\begin{array}{cc}
\beta_{1, i-1}\left(\psi_{i-1}\right) & \beta_{2, i-1}\left(\psi_{i-1}\right) \\
\beta_{1, i-1}^{\prime}\left(\psi_{i-1}\right) & \beta_{2, i-1}^{\prime}\left(\psi_{i-1}\right)
\end{array}\right)=\left(\begin{array}{cc}
1 & 0 \\
0 & 1
\end{array}\right)
$$

Suppose $W_{1, i-1}, W_{2, i-1}$ solves

$$
\mathcal{L}_{\tau(0)} W_{j, i-1}=0, \psi \in\left[\tilde{\psi}_{i-1}, \tilde{\psi}_{i}\right], j=1,2,
$$

with

$$
\left(\begin{array}{ll}
W_{1, i-1}\left(\tilde{\psi}_{i-1}\right) & W_{2, i-1}\left(\tilde{\psi}_{i-1}\right) \\
W_{1, i-1}^{\prime}\left(\tilde{\psi}_{i-1}\right) & W_{2, i-1}^{\prime}\left(\tilde{\psi}_{i-1}\right)
\end{array}\right)=\left(\begin{array}{cc}
1 & 0 \\
0 & 1
\end{array}\right)
$$


We know

$$
\begin{aligned}
& W_{1, i-1}(\varsigma)=h_{1}(\tau(0))\left(\varsigma-\tilde{\psi}_{i-1}\right) \frac{\partial \phi_{\tau(0)}}{\partial \varsigma}+v_{\tau(0)}(\varsigma), \\
& W_{2, i-1}(\varsigma)=h_{2}(\tau(0)) \frac{\partial \phi_{\tau(0)}}{\partial \varsigma}
\end{aligned}
$$

where $v_{\tau(0)}(\varsigma)$ has period $\tilde{\psi}_{1}$.

Comparing the coefficients of $\mathcal{L}_{\xi, \mu, \omega, \tau(0)}$ with that of $\mathcal{L}_{\tau(0)}$ we have

$$
\begin{aligned}
& \quad\left|\beta_{j, i-1}\left((1-\lambda) \psi_{i-1}+\lambda \psi_{i}\right)-W_{j, i-1}\left((1-\lambda) \tilde{\psi}_{i-1}+\lambda \tilde{\psi}_{i}\right)\right| \\
& \quad+\left|\beta_{j, i-1}^{\prime}\left((1-\lambda) \psi_{i-1}+\lambda \psi_{i}\right)-W_{j, i-1}^{\prime}\left((1-\lambda) \tilde{\psi}_{i-1}+\lambda \tilde{\psi}_{i}\right)\right| \\
& \leq C\left(\tau_{0}\right)\left(1+C_{1}+C_{2}+C_{3}\right) \varepsilon^{2}
\end{aligned}
$$

which implies the following lemma,

Lemma 3.14.

$$
\begin{aligned}
& \quad\left\|\beta_{1, i-1}(\psi)-\left(h_{1}(\tau(0))\left(\psi-\psi_{i}\right) \frac{\partial \phi}{\partial \psi}+v_{1}(\psi)\right)\right\|_{C_{\varepsilon}^{1}\left(\left[\psi_{i-1}, \psi_{i}\right]\right)} \\
& \quad+\left\|\beta_{2, i-1}(\psi)-h_{2}(\tau(0)) \frac{\partial \phi}{\partial \psi}\right\|_{C_{\varepsilon}^{1}\left(\left[\psi_{i-1}, \psi_{i}\right]\right)} \\
& \leq C\left(\tau_{0}\right)\left(1+C_{1}+C_{2}+C_{3}\right) \varepsilon^{2}
\end{aligned}
$$

where $v_{1}\left((1-\lambda) \psi_{i-1}+\lambda \psi_{i}\right)=v_{\tau(0)}\left(\lambda\left(\tilde{\psi}_{i}-\tilde{\psi}_{i-1}\right)\right)$.

From Lemma 3.14 we have

$$
\left(\begin{array}{cc}
\beta_{1, i-1}\left(\psi_{i}\right) & \beta_{2, i-1}\left(\psi_{i}\right) \\
\frac{\partial \beta_{1, i-1}}{\partial \psi}\left(\psi_{i}\right) & \frac{\partial \beta_{2, i-1}}{\partial \psi}\left(\psi_{i}\right)
\end{array}\right)=\left(\begin{array}{cc}
1+e_{11}^{i} & e_{12}^{i} \\
\kappa+e_{21}^{i} & 1+e_{22}^{i}
\end{array}\right),
$$

where $\left|e_{j k}^{i}\right| \leq C\left(\tau_{0}\right)\left(1+C_{1}+C_{2}+C_{3}\right) \varepsilon^{2}$.

From the theory of linear ODE, we know

$$
\begin{aligned}
\left(\begin{array}{cc}
\beta_{1}\left(\psi_{i}\right) & \beta_{2}\left(\psi_{i}\right) \\
\frac{\partial \beta_{1}}{\partial \psi}\left(\psi_{i}\right) & \frac{\partial \beta_{2}}{\partial \psi}\left(\psi_{i}\right)
\end{array}\right) & =\left(\begin{array}{cc}
1+e_{11}^{i} & e_{12}^{i} \\
\kappa+e_{21}^{i} & 1+e_{22}^{i}
\end{array}\right) \cdots\left(\begin{array}{cc}
1+e_{11}^{1} & e_{12}^{1} \\
\kappa+e_{21}^{1} & 1+e_{22}^{1}
\end{array}\right) \\
& =\left(\begin{array}{cc}
A_{11}^{i} & A_{12}^{i} \\
A_{21}^{i} & A_{22}^{i}
\end{array}\right) .
\end{aligned}
$$

By analysis of the matrix, we can prove

Lemma 3.15.

$$
\begin{aligned}
\exp \left(-C\left(\tau_{0}\right)\left(1+C_{1}+C_{2}+C_{3}\right)\right) & \leq A_{11}^{i}+A_{22}^{i}+\varepsilon A_{21}^{i} \leq \exp C\left(\tau_{0}\right)\left(1+C_{1}+C_{2}+C_{3}\right), \\
\left|A_{12}^{i}\right| & \leq C\left(\tau_{0}, C_{1}, C_{2}, C_{3}\right) \varepsilon \exp C\left(\tau_{0}\right)\left(1+C_{1}+C_{2}+C_{3}\right) .
\end{aligned}
$$

We will prove this result in Appendix D So from Lemma 3.14 we have 
Lemma 3.16. For $\xi, \mu, \omega, \tau(0)$ which satisfy (28), there is $\delta>0$ such that when $0<\varepsilon<\delta$, in the interval $\left[\psi_{i}, \psi_{i+1}\right]$, we have

$$
\begin{aligned}
& \left\|\beta_{1}(\psi)-\left(A_{11}^{i}\left[h_{1}(\tau(0))\left(\psi-\psi_{i}\right) \frac{\partial \phi}{\partial \psi}+v_{i}(\psi)\right]+A_{21}^{i} h_{2}(\tau(0)) \frac{\partial \phi}{\partial \psi}\right)\right\|_{C_{\varepsilon}^{1}} \\
\leq & C\left(\tau_{0}, C_{1}, C_{2}, C_{3}\right) \varepsilon, \\
& \left\|\beta_{2}(\psi)-\left(A_{12}^{i}\left[h_{1}(\tau(0))\left(\psi-\psi_{i}\right) \frac{\partial \phi}{\partial \psi}+v_{i}(\psi)\right]+A_{22}^{i} h_{2}(\tau(0)) \frac{\partial \phi}{\partial \psi}\right)\right\|_{C_{\varepsilon}^{1}} \\
\leq & C\left(\tau_{0}, C_{1}, C_{2}, C_{3}\right) \varepsilon^{2} .
\end{aligned}
$$

where $v_{i}\left((1-\lambda) \psi_{i}+\lambda \psi_{i+1}\right)=v_{\tau(0)}\left((1-\lambda) \Phi\left(\psi_{i}\right)+\lambda \Phi\left(\psi_{i+1}\right)\right)$ for $\lambda \in[0,1]$ and $A_{k l}^{i}$ satisfy the inequalities in Lemma 3.15 .

In particular, $\beta_{1}$ has linear growth and $\beta_{2}$ is bounded.

We denote

$$
\begin{cases}\left.\frac{d}{d t} \phi_{\xi, \mu+t \Delta \mu, \omega, \tau(0)}(\psi)\right|_{t=0} & =\beta_{\mu}(\psi), \\ \left.\frac{d}{d t} \phi_{\xi+t \Delta \xi, \mu, \omega, \tau(0)}(\psi)\right|_{t=0} & =\beta_{\xi}(\psi), \\ \left.\frac{d}{d t} \phi_{\xi, \mu, \omega+t, \tau(0)}(\psi)\right|_{t=0} & =\beta_{\omega}(\psi) .\end{cases}
$$

Using (43), Lemma 3.15, 3.16, we have the following estimates

\section{Lemma 3.17.}

$$
\begin{aligned}
\left\|\beta_{\mu}(\psi)\right\|_{C_{\varepsilon}^{1}} & \leq C\left(\tau_{0}, C_{1}, C_{2}, C_{3}\right) \varepsilon\|\Delta \mu\|_{C^{0}} \\
\left\|\beta_{\xi}(\psi)\right\|_{C_{\varepsilon}^{1}} & \leq C\left(\tau_{0}, C_{1}, C_{2}, C_{3}\right)\|\Delta \xi\|_{C_{x_{0}}^{1}} \\
\left\|\beta_{\omega}(\psi)\right\|_{C_{\varepsilon}^{1}} & \leq C\left(\tau_{0}, C_{1}, C_{2}, C_{3}\right) \varepsilon
\end{aligned}
$$

Moreover if we consider

$$
\frac{\partial\left(\Delta \tau, \zeta\left(\frac{L_{\Gamma}}{\varepsilon}\right)\right)}{\partial(\omega, \phi(0))}
$$

where $\Delta \tau=\tau\left(\frac{L_{\Gamma}}{\varepsilon}\right)-\tau(0)$ and $\zeta=\phi_{\psi}$. We can get

$$
\begin{cases}\frac{\partial \Delta \tau}{\partial \omega} & \geq C_{5}\left(\tau_{0}\right) \varepsilon^{2} \\ \left|\frac{\partial \Delta \tau}{\partial \phi(0)}\right| & \leq K_{1}\left(\tau_{0}, C_{1}, C_{2}, C_{3}\right) \varepsilon \\ \left|\frac{\partial \zeta\left(\frac{L_{\Gamma}}{\varepsilon}\right)}{\partial \omega}\right| & =\left|\beta_{\omega}^{\prime}\left(\frac{L_{\Gamma}}{\varepsilon}\right)\right| \leq K_{2}\left(\tau_{0}, C_{1}, C_{2}, C_{3}\right) \varepsilon \\ \frac{\partial \zeta\left(\frac{L_{\Gamma}}{\varepsilon}\right)}{\partial \phi(0)} & =\beta_{1}^{\prime}\left(\frac{L_{\Gamma}}{\varepsilon}\right) \geq \exp \left(-C\left(\tau_{0}\right)\left(1+C_{1}+C_{2}+C_{3}\right)\right) \frac{1}{\varepsilon} .\end{cases}
$$

We will prove this lemma in Appendix E We note that the proof of (47) relies on Lemma 3.6, the "average 0" lemma.

\section{Step 5. Match the boundary value}


Lemma 3.18. For $C_{1}, C_{2}>0$, if $\|\xi\|_{C_{x_{0}}^{1}} \leq C_{1} \varepsilon^{2}$ and $\|\mu\|_{C_{\varepsilon}^{\alpha}} \leq C_{2}$, we can choose $C_{3}, C_{4}$ and $\delta$ such that when $0<\varepsilon<\delta$, there are unique $\omega_{\xi, \mu}$ and $\phi(0)_{\xi, \mu}$ (or $\tau(0)_{\xi, \mu}$ ) such that

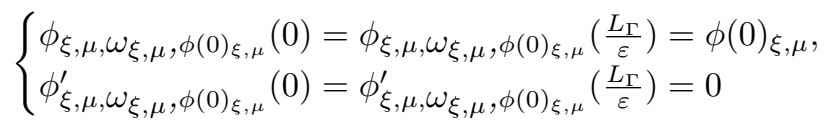

and

$$
\begin{aligned}
\left|\omega_{\xi, \mu}\right| & \leq C_{3}, \\
\left|\phi(0)_{\xi, \mu}-\frac{1-\sqrt{1-4 \tau_{0}}}{2}\right| & \leq C_{4} \varepsilon^{2} .
\end{aligned}
$$

Proof. We begin with $\omega=0, \tau(0)=\tau_{0}$. From (37) and Corollary 3.12 we have for a particular $\tilde{C}=\tilde{C}\left(\tau_{0}\right)$

$$
\begin{aligned}
|\Delta \tau| & =\left|\tau\left(\frac{L_{\Gamma}}{\varepsilon}\right)-\tau(0)\right| \leq \tilde{C}\left(\tau_{0}\right) \varepsilon^{2}\left(1+C_{1}+C_{2}\right) \\
\left|\zeta\left(\frac{L_{\Gamma}}{\varepsilon}\right)\right| & \leq \tilde{C}\left(\tau_{0}\right) \varepsilon\left(1+C_{1}+C_{2}\right) .
\end{aligned}
$$

We choose

$$
C_{3}=\frac{4 \tilde{C}\left(\tau_{0}\right)\left(1+C_{1}+C_{2}\right)}{C_{5}\left(\tau_{0}\right)}
$$

and

$$
C_{4}=4 \tilde{K}_{2} C_{5}^{-1} \tilde{C}\left(\tau_{0}\right)\left(1+C_{1}+C_{2}\right) \exp \left(C\left(\tau_{0}\right)\left(1+C_{1}+C_{2}+C_{3}\right)\right)
$$

where $\tilde{K}_{2}=\tilde{K}_{2}\left(\tau_{0}, C_{1}, C_{2}, C_{3}, C_{5}\right)=K_{2}\left(\tau_{0}, C_{1}, C_{2}, C_{3}\right)+C_{5}$. We choose

$$
0<\varepsilon \leq C_{6}=\min \left\{\frac{C\left(\tau_{0}\right)}{C_{4}}, \frac{C_{5} \exp \left(-C\left(\tau_{0}\right)\left(1+C_{1}+C_{2}+C_{3}\right)\right)}{2\left|K_{1} \tilde{K}_{2}\right|+1}\right\} .
$$

Note that first $C_{4} \varepsilon^{2} \leq C\left(\tau_{0}\right) \varepsilon$, so one can work with a uniform constant $C_{5}\left(\tau_{0}\right)>0$ such that

$$
\frac{\partial \Delta \tau}{\partial \omega} \geq C_{5} \varepsilon^{2}
$$

First we prove the uniqueness. For $\omega_{1}, \omega_{2} \in\left[-C_{3}, C_{3}\right], \phi(0)_{1}, \phi(0)_{2} \in\left[\frac{1-\sqrt{1-4 \tau_{0}}}{2}-\right.$ $\left.C_{4} \varepsilon^{2}, \frac{1-\sqrt{1-4 \tau_{0}}}{2}+C_{4} \varepsilon^{2}\right]$, if $\Delta \tau$ and $\zeta\left(\frac{L_{\Gamma}}{\varepsilon}\right)$ takes the same value at $\left(\omega_{1}, \phi(0)_{1}\right)$ and $\left(\omega_{2}, \phi(0)_{2}\right)$ then there exists $\omega_{3}, \omega_{4}$ lying between $\omega_{1}, \omega_{2}$ and $\phi(0)_{3}, \phi(0)_{4}$ lying between $\phi(0)_{1}, \phi(0)_{2}$ such that

$$
\left[\begin{array}{cc}
\left.\frac{\partial \Delta \tau}{\partial \omega}\right|_{\left(\omega_{3}, \phi(0)_{1}\right)} & \left.\frac{\partial \Delta \tau}{\partial \phi(0)}\right|_{\left(\omega_{2}, \phi(0)_{3}\right)} \\
\left.\frac{\partial \zeta\left(\frac{L_{\Gamma}}{\varepsilon}\right)}{\partial \omega}\right|_{\left(\omega_{4}, \phi(0)_{1}\right)} & \left.\frac{\partial \zeta\left(\frac{(\Gamma}{\varepsilon}\right)}{\partial \phi(0)}\right|_{\left(\omega_{2}, \phi(0)_{4}\right)}
\end{array}\right]\left[\begin{array}{c}
\omega_{1}-\omega_{2} \\
\phi(0)_{1}-\phi(0)_{2}
\end{array}\right]=\left[\begin{array}{l}
0 \\
0
\end{array}\right] .
$$

From estimate 494 , the matrix $\left[\begin{array}{cc}\left.\frac{\partial \Delta \tau}{\partial \omega}\right|_{\left(\omega_{3}, \phi(0)_{1}\right)} & \left.\frac{\partial \Delta \tau}{\partial \phi(0)}\right|_{\left(\omega_{2}, \phi(0)_{3}\right)} \\ \frac{\partial \zeta\left(\left.\frac{\left.L_{\Gamma}\right)}{\varepsilon}\right|_{\left(\omega_{4}, \phi(0)_{1}\right)}\right.}{\partial \omega} & \left.\frac{\partial \zeta\left(\frac{L}{\varepsilon}\right)}{\partial \phi(0)}\right|_{\left(\omega_{2}, \phi(0)_{4}\right)}\end{array}\right]$ is invertible as long as $\varepsilon \leq C_{6}$. So $\omega_{1}=\omega_{2}$ and $\phi(0)_{1}=\phi(0)_{2}$. We have proved the uniqueness. 
For the existence first we perturb $\omega$ within $\frac{\tilde{C}\left(1+C_{1}+C_{2}\right)}{C_{5}}$ such that $\Delta \tau=0$. Then $\zeta\left(\frac{L_{\Gamma}}{\varepsilon}\right)$ will change no more than $K_{2} \frac{\tilde{C}\left(1+C_{1}+C_{2}\right)}{C_{5}} \varepsilon$. Then we perturb $\phi(0)$ within

$$
\tilde{K}_{2} C_{5}^{-1} \tilde{C}\left(1+C_{1}+C_{2}\right) \exp \left(C\left(\tau_{0}\right)\left(1+C_{1}+C_{2}+C_{3}\right)\right) \varepsilon^{2}
$$

such that $\zeta\left(\frac{L_{\Gamma}}{\varepsilon}\right)=0$. Then $\Delta \tau$ will change no more than $K_{1} \tilde{K}_{2} C_{5}^{-1} \tilde{C}\left(1+C_{1}+\right.$ $\left.C_{2}\right) \exp \left(C\left(\tau_{0}\right)\left(1+C_{1}+C_{2}+C_{3}\right)\right) \varepsilon^{3}$. From

$$
\varepsilon \leq \frac{C_{5} \exp \left(-C\left(\tau_{0}\right)\left(1+C_{1}+C_{2}+C_{3}\right)\right)}{2\left|K_{1} \tilde{K}_{2}\right|+1}
$$

we know

$$
\begin{aligned}
& K_{1} \tilde{K}_{2} C_{5}^{-1} \tilde{C}\left(1+C_{1}+C_{2}\right) \exp \left(C\left(\tau_{0}\right)\left(1+C_{1}+C_{2}+C_{3}\right)\right) \varepsilon^{3} \\
\leq & \frac{1}{2} \tilde{C}\left(1+C_{1}+C_{2}\right) \varepsilon^{2} .
\end{aligned}
$$

Then by an iteration argument, we have, there exists $\left|\omega_{\xi, \mu}\right| \leq C_{3}$ and $\mid \phi(0)_{\xi, \mu}-$ $\frac{1-\sqrt{1-4 \tau_{0}}}{2} \mid \leq C_{4} \varepsilon^{2}$ such that $\Delta \tau=0$ and $\zeta\left(\frac{L_{\Gamma}}{\varepsilon}\right)=0$. From $\mid \phi_{\xi, \mu, \omega_{\xi, \mu}, \tau(0)_{\xi, \mu}\left(\frac{L_{\Gamma}}{\varepsilon}\right)-}$

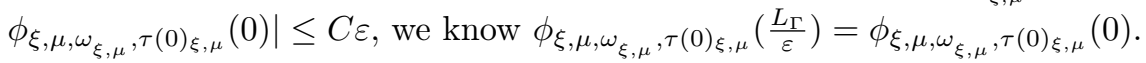

From Corollary 3.12 and Lemma 3.18 we have

Corollary 3.19. For given $C_{1}, C_{2}$ and $\tau_{0}$ there is $C=C\left(\tau_{0}, C_{1}, C_{2}\right)$ such that

$$
\left\|\phi_{\xi, \mu}(\psi)-\phi_{\tau_{0}}(\psi)\right\|_{C_{\varepsilon}^{2, \alpha}} \leq C \varepsilon .
$$

As $\left|\tau(0)_{\xi, \mu}-\tau_{0}\right| \leq C \varepsilon^{2}$, we know $\left(\phi_{\xi, \mu}, \zeta_{\xi, \mu}\right)$ is within $C \varepsilon^{2}$ neighborhood of $\left(\phi_{\tau_{0}}, \zeta_{\tau_{0}}\right)$. So similar to the construction of $\Phi$, we can construct $\tilde{\Phi}$ by projecting $\left(\phi_{\xi, \mu}, \zeta_{\xi, \mu}\right)$ to the orbit $\left(\phi_{\tau_{0}}, \zeta_{\tau_{0}}\right)$. We have

Corollary 3.20. For $\phi_{\xi, \mu, \omega_{\xi, \mu} \phi(0)_{\xi, \mu}}(\psi)$ we have a $C^{1}$ map

$$
\tilde{\Phi}=\tilde{\Phi}_{\xi, \mu, \tau_{0}}: \Gamma \rightarrow \Gamma
$$

such that

$$
\begin{aligned}
& \left|\phi_{\xi, \mu, \omega_{\xi, \mu} \phi(0)_{\xi, \mu}}(\psi)-\phi_{\tau_{0}}(\tilde{\Phi}(\psi))\right|+\left|\zeta_{\xi, \mu, \omega_{\xi, \mu} \phi(0)_{\xi, \mu}}(\psi)-\zeta_{\tau_{0}}(\tilde{\Phi}(\psi))\right| \\
\leq & C\left(\tau_{0}, C_{1}, C_{2}\right) \varepsilon^{2}
\end{aligned}
$$

and

$$
\left\{\begin{array}{l}
|\tilde{\Phi}(\psi)-\psi| \leq C\left(\tau_{0}, C_{1}, C_{2}\right) \varepsilon, \\
\left|\tilde{\Phi}^{\prime}(\psi)-1\right| \leq C\left(\tau_{0}, C_{1}, C_{2}\right) \varepsilon^{2} .
\end{array}\right.
$$

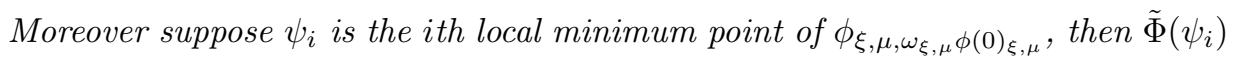
is the ith local minimum point of $\phi_{\tau_{0}}$. In particular $\tilde{\Phi}(0)=0, \tilde{\Phi}\left(L_{\Gamma}\right)=L_{\Gamma}$. 
Step 6. Main estimates of 0th mode For

$$
\left\|\xi_{1}\right\|_{C_{x_{0}}^{1}},\left\|\xi_{2}\right\|_{C_{x_{0}}^{1}} \leq C_{1} \varepsilon^{2},\left\|\mu_{1}\right\|_{C_{\varepsilon}^{\alpha}},\left\|\mu_{2}\right\|_{C_{\varepsilon}^{\alpha}} \leq C_{2}
$$

we have

$$
\begin{gathered}
\quad\left\|\phi_{\xi_{1}, \mu_{1}, \omega_{\xi_{1}, \mu_{1}}, \tau(0)_{\xi_{1}, \mu_{1}}}(\psi)-\phi_{\xi_{2}, \mu_{2}, \omega_{\xi_{2}, \mu_{2}}, \tau(0)_{\xi_{2}, \mu_{2}}}(\psi)\right\|_{C_{\varepsilon}^{1}} \\
\leq\left\|\phi_{\xi_{1}, \mu_{1}, \omega_{\xi_{1}, \mu_{1}, \tau(0)}}(\psi)_{\xi_{1}, \mu_{1}}(\psi)-\phi_{\left.\xi_{2}, \mu_{2}, \omega_{\xi_{1}, \mu_{1}, \tau(0)},\right)_{\xi_{1}, \mu_{1}}}(\psi)\right\|_{C_{\varepsilon}^{1}} \\
\quad+\left\|\phi_{\xi_{2}, \mu_{2}, \omega_{\xi_{1}, \mu_{1}}, \tau(0)_{\xi_{1}, \mu_{1}}}(\psi)-\phi_{\xi_{2}, \mu_{2}, \omega_{\xi_{2}, \mu_{2}}, \tau(0)_{\xi_{2}, \mu_{2}}}(\psi)\right\|_{C_{\varepsilon}^{1}} .
\end{gathered}
$$

Note that from (46) and (47) we have

$$
\begin{aligned}
& \left\|\phi_{\xi_{1}, \mu_{1}, \omega_{\xi_{1}, \mu_{1}}, \tau(0) \xi_{\xi_{1}, \mu_{1}}}(\psi)-\phi_{\xi_{2}, \mu_{2}, \omega_{\xi_{1}, \mu_{1}}, \tau(0)_{\xi_{1}, \mu_{1}}}(\psi)\right\|_{C_{\varepsilon}^{1}} \\
\leq & \int_{0}^{1}\left\|\frac{d}{d t} \phi_{t \xi_{1}+(1-t) \xi_{2}, \mu_{1}, \omega_{\xi_{1}, \mu_{1}}, \tau(0)_{\xi_{1}, \mu_{1}}}(\psi)\right\|_{C_{\varepsilon}^{1}} d \psi \\
& +\int_{0}^{1}\left\|\frac{d}{d t} \phi_{\xi_{2}, t \mu_{1}+(1-t) \mu_{2}, \omega_{\xi_{1}, \mu_{1}}, \tau(0)_{\xi_{1}, \mu_{1}}}(\psi)\right\|_{C_{\varepsilon}^{1}} d \psi \\
\leq & C \varepsilon\left\|\mu_{2}-\mu_{1}\right\|_{C_{\varepsilon}^{\alpha}}+C\left\|\xi_{2}-\xi_{1}\right\|_{C_{x_{0}}^{1}} .
\end{aligned}
$$

By comparing $\phi_{\xi_{2}, \mu_{2}, \omega_{\xi_{1}, \mu_{1}}, \tau(0)_{\xi_{1}, \mu_{1}}}(\psi)$ with $\phi_{\xi_{1}, \mu_{1}, \omega_{\xi_{1}, \mu_{1}}, \tau(0)_{\xi_{1}, \mu_{1}}}(\psi)$, we have for $\phi_{\xi_{2}, \mu_{2}, \omega_{\xi_{1}, \mu_{1}}, \tau(0)_{\xi_{1}, \mu_{1}}}(\psi)$

$$
\begin{aligned}
|\Delta \tau| & \leq C\left(\tau_{0}, C_{1}, C_{2}, C_{3}\right)\left(\varepsilon^{2}\left\|\mu_{2}-\mu_{1}\right\|_{C_{\varepsilon}^{\alpha}}+\varepsilon\left\|\xi_{2}-\xi_{1}\right\|_{C_{x_{0}}^{1}}\right), \\
\left|\zeta\left(\frac{L_{\Gamma}}{\varepsilon}\right)\right| & \leq C\left(\tau_{0}, C_{1}, C_{2}, C_{3}\right)\left(\varepsilon\left\|\mu_{2}-\mu_{1}\right\|_{C_{\varepsilon}^{\alpha}}+\left\|\xi_{2}-\xi_{1}\right\|_{C_{x_{0}}^{1}}\right) .
\end{aligned}
$$

If we denote

$$
\left(\begin{array}{ll}
B_{11} & B_{12} \\
B_{21} & B_{22}
\end{array}\right)=\frac{\partial\left(\Delta \tau, \zeta\left(\frac{L_{\Gamma}}{\varepsilon}\right)\right)^{-1}}{\partial(\omega, \phi(0))}
$$

we can get

$$
\begin{aligned}
& \left|B_{11}\right| \leq 2 C_{5}^{-1} \varepsilon^{-2} \\
& \left|B_{22}\right| \leq 2 \exp \left(C\left(\tau_{0}\right)\left(1+C_{1}+C_{2}+C_{3}\right)\right) \varepsilon \\
& \left|B_{12}\right| \leq 2 K_{1} C_{5}^{-1} \exp \left(C\left(\tau_{0}\right)\left(1+C_{1}+C_{2}+C_{3}\right)\right) \\
& \left|B_{21}\right| \leq 2 K_{2} C_{5}^{-1} \exp \left(C\left(\tau_{0}\right)\left(1+C_{1}+C_{2}+C_{3}\right)\right) .
\end{aligned}
$$

So we know for some $C=C\left(\tau_{0}, C_{1}, C_{2}, C_{3}\right)$

$$
\begin{cases}\left|\omega_{\xi_{2}, \mu_{2}}-\omega_{\xi_{1}, \mu_{1}}\right| & \leq \frac{C}{\varepsilon}\left(\varepsilon\left\|\mu_{2}-\mu_{1}\right\|_{C_{\varepsilon}^{\alpha}}+\left\|\xi_{2}-\xi_{1}\right\|_{C_{x_{0}}^{1}}\right) \\ \left|\phi(0)_{\xi_{2}, \mu_{2}}-\phi(0)_{\xi_{1}, \mu_{1}}\right| & \leq C\left(\varepsilon^{2}\left\|\mu_{2}-\mu_{1}\right\|_{C_{\varepsilon}^{\alpha}}+\varepsilon\left\|\xi_{2}-\xi_{1}\right\|_{C_{x_{0}}^{1}}\right)\end{cases}
$$

So from (48) and Lemma 3.16 we have

$$
\begin{aligned}
& \left|\phi_{\xi_{2}, \mu_{2}, \omega_{\xi_{2}, \mu_{2}}, \tau(0)_{\xi_{2}, \mu_{2}}}(\psi)-\phi_{\xi_{1}, \mu_{1}, \omega_{\xi_{1}, \mu_{1}}, \tau(0)_{\xi_{1}, \mu_{1}}}(\psi)\right| \\
& \left.+\mid \zeta_{\xi_{2}, \mu_{2}, \omega_{\xi_{2}, \mu_{2}, \tau(0)}}(\psi)\right)_{\xi_{2}, \mu_{2}}(\psi)-\zeta_{\left.\xi_{1}, \mu_{1}, \omega_{\xi_{1}, \mu_{1}}, \tau(0)\right)_{\xi_{1}, \mu_{1}}}(\psi) \mid \\
\leq & C\left(\varepsilon\left\|\mu_{2}-\mu_{1}\right\|_{C_{\varepsilon}^{\alpha}}+\left\|\xi_{2}-\xi_{1}\right\|_{C_{x_{0}}^{1}}^{1}\right) .
\end{aligned}
$$


And from 27)

$$
\begin{aligned}
& \left\|\phi_{\xi_{2}, \mu_{2}, \omega_{\xi_{2}, \mu_{2}, \tau(0)}, \xi_{\xi_{2}, \mu_{2}}}(\psi)-\phi_{\xi_{1}, \mu_{1}, \omega_{\xi_{1}, \mu_{1}}, \tau(0) \xi_{\xi_{1}, \mu_{1}}}(\psi)\right\|_{C_{\varepsilon}^{2, \alpha}} \\
\leq & C\left(\varepsilon\left\|\mu_{2}-\mu_{1}\right\|_{C_{\varepsilon}^{\alpha}}+\left\|\xi_{2}-\xi_{1}\right\|_{C_{x_{0}}^{1}}\right) .
\end{aligned}
$$

At last note $C_{3}=C_{3}\left(\tau_{0}, C_{1}, C_{2}\right)$. So $C\left(\tau_{0}, C_{1}, C_{2}, C_{3}\right)=C\left(\tau_{0}, C_{1}, C_{2}\right)$.

\section{The existence of CMC surfaces}

\subsection{A fixed point argument}

In 0th mode, if we prescribe $\xi \in C_{x_{0}}^{1}, \mu \in C_{\varepsilon}^{\alpha}, \omega_{\xi, \mu}$ and $\phi(0)_{\xi, \mu}$ in $\rho$, we can get $\phi_{\xi, \mu, \omega_{\xi, \mu}, \phi(0)_{\xi, \mu}}$, which we denote by $\phi_{\xi, \mu}$ for short. We define parameter $s$ using $\dot{\psi}=\frac{d \psi}{d s}=\phi_{\xi, \mu}^{2}+\tau_{\xi, \mu}$ where $\tau_{\xi, \mu}=-\phi_{\xi, \mu}^{2}+\frac{\phi_{\xi, \mu}}{\sqrt{1+\zeta_{\xi, \mu}^{2}}}$. From 27 we know

$$
\dot{\phi}_{\xi, \mu}^{2}+\left(\phi_{\xi, \mu}^{2}+\tau_{\xi, \mu}\right)^{2}=\phi_{\xi, \mu}^{2} .
$$

And further we have

$$
\begin{aligned}
& \ddot{\phi}_{\xi, \mu}=\phi_{\xi, \mu}-(2+\rho) \phi_{\xi, \mu}\left(\phi_{\xi, \mu}^{2}+\tau_{\xi, \mu}\right), \\
& \ddot{\psi}_{\xi, \mu}=\phi_{\xi, \mu} \dot{\phi}_{\xi, \mu}(2+\rho) .
\end{aligned}
$$

Follow the calculations of mean curvature in Appendix A and we can get $H\left(\mathcal{D}_{\phi_{\xi, \mu}, p_{0}, \varepsilon}(w, \eta)\right)$. We substitute

$$
\frac{\dot{\phi}_{\xi, \mu} \ddot{\psi}}{\phi_{\xi, \mu}}-\frac{\ddot{\phi}_{\xi, \mu} \dot{\psi}}{\phi_{\xi, \mu}}=\phi_{\xi, \mu}^{2}-\tau_{\xi, \mu}+\phi_{\xi, \mu}^{2} \rho
$$

for 600 .

Definition 4. In the following, $L_{\xi, \mu}(\tilde{w}, \eta)$ denotes any expression which is linear differential operator (of order at most 2), which satisfies

$$
\begin{aligned}
\left\|L_{\xi, \mu}(\tilde{w}, \eta)\right\|_{C_{\varepsilon}^{\alpha}} \leq & C\left(\|\tilde{w}\|_{C_{\varepsilon}^{2, \alpha}(S N \Gamma)}+\|\eta\|_{C_{x_{0}, \varepsilon}^{2, \alpha}(\Gamma, N \Gamma)}\right) \\
\left\|L_{\xi_{1}, \mu_{1}}(\tilde{w}, \eta)-L_{\xi_{2}, \mu_{2}}(\tilde{w}, \eta)\right\|_{C_{\varepsilon}^{\alpha} \leq} & C\left(\varepsilon\left\|\mu_{1}-\mu_{2}\right\|_{C_{\varepsilon}^{\alpha}}+\left\|\xi_{1}-\xi_{2}\right\|_{C_{x_{0}}^{1}}\right) \\
& \times\left(\|\tilde{w}\|_{C_{\varepsilon}^{2, \alpha}(S N \Gamma)}+\|\eta\|_{C_{x_{0}, \varepsilon}^{2, \alpha}(\Gamma, N \Gamma)}\right) .
\end{aligned}
$$

$Q_{\xi, \mu}(\tilde{w}, \eta)$ denotes any nonlinear differential operator (of order less than or equal to 2$)$ in $\tilde{w}$ and $\eta$ which vanishes quadratically in the pair $(\tilde{w}, \eta)$ and such that

$$
\begin{aligned}
\left\|Q_{\xi, \mu}\left(\tilde{w}_{1}, \eta_{1}\right)-Q_{\xi, \mu}\left(\tilde{w}_{2}, \eta_{2}\right)\right\|_{C_{\varepsilon}^{\alpha}} \leq & C \sup _{i=1,2}\left(\left\|\tilde{w}_{i}\right\|_{C_{\varepsilon}^{2, \alpha}(S N \Gamma)}+\left\|\eta_{i}\right\|_{C_{x_{0}, \varepsilon}^{2, \alpha}(\Gamma, N \Gamma)}\right) \\
& \times\left(\left\|\tilde{w}_{1}-\tilde{w}_{2}\right\|_{C_{\varepsilon}^{2, \alpha}(S N \Gamma)}+\left\|\eta_{1}-\eta_{2}\right\|_{C_{x_{0}, \varepsilon}^{2, \alpha}(\Gamma, N \Gamma)}\right), \\
\left\|Q_{\xi_{1}, \mu_{1}}(\tilde{w}, \eta)-Q_{\xi_{2}, \mu_{2}}(\tilde{w}, \eta)\right\|_{C_{\varepsilon}^{\alpha} \leq} & C\left(\varepsilon\left\|\mu_{1}-\mu_{2}\right\|_{C_{\varepsilon}^{\alpha}}+\left\|\xi_{1}-\xi_{2}\right\|_{C_{x_{0}}^{1}}\right) \\
& \times\left(\|\tilde{w}\|_{C_{\varepsilon}^{2, \alpha}(S N \Gamma)}^{2}+\|\eta\|_{C_{x_{0}, \varepsilon}^{2, \alpha}(\Gamma, N \Gamma)}^{2}\right) .
\end{aligned}
$$


Finally $E_{\xi, \mu}=E\left(\phi_{\xi, \mu}, \frac{\partial \phi_{\xi, \mu}}{\partial \psi}, \psi\right)$, where $E$ is defined in Definition 2. For $E_{\xi, \mu}$, we have

$$
\left\|E_{\xi_{1}, \mu_{1}}-E_{\xi_{2}, \mu_{2}}\right\| \leq C\left(\varepsilon\left\|\mu_{1}-\mu_{2}\right\|_{C_{\varepsilon}^{\alpha}}+\left\|\xi_{1}-\xi_{2}\right\|_{C_{x_{0}}^{1}}\right) .
$$

Note that each time when $\ddot{\phi}, \ddot{\psi}$ appear, one term $\rho$ comes out. Then, letting $\zeta_{\xi, \mu}=\partial_{\psi} \phi_{\xi, \mu}$, we have,

$$
\begin{aligned}
H\left(\mathcal{D}_{\phi_{\xi, \mu}, p_{0}, \varepsilon}(\tilde{w}, \eta)\right)= & \frac{2}{\varepsilon}+\frac{\rho}{\varepsilon}+\frac{1}{\varepsilon} \tilde{\mathcal{L}}_{\xi, \mu} \tilde{w}+<\mathcal{J}_{\xi, \mu} \eta, \Upsilon> \\
& +\varepsilon\left(F_{1}\left(\phi_{\xi, \mu}, \zeta_{\xi, \mu}\right) \star R_{1}+F_{2}\left(\phi_{\xi, \mu}, \zeta_{\xi, \mu}\right) \star R_{2}\right) \\
& +\left(\varepsilon^{2}+\varepsilon \rho\right) E_{\xi, \mu}+F_{3}\left(\phi_{\xi, \mu}, \zeta_{\xi, \mu}\right) \star R_{3}(\eta) \\
& +\left(\varepsilon+\varepsilon^{-1} \rho\right) L_{\xi, \mu}(\tilde{w}, \eta)+\varepsilon^{-1}(1+\rho) Q_{\xi, \mu}(\tilde{w}, \eta)
\end{aligned}
$$

where

$$
\begin{aligned}
\tilde{\mathcal{L}}_{\xi, \mu} \tilde{w}= & -\frac{\dot{\psi}_{\xi, \mu}}{\phi_{\xi, \mu}^{3}}\left(\frac{\partial^{2}}{\partial s^{2}}+\frac{\partial^{2}}{\partial \theta^{2}}\right) \tilde{w}-\left(\phi_{\xi, \mu}^{2}-\tau_{\xi, \mu}\right) \frac{\dot{\phi}_{\xi, \mu}}{\phi_{\xi, \mu}^{4}} \frac{\partial w}{\partial s}-\frac{\dot{\psi}_{\xi, \mu}}{\phi_{\xi, \mu}^{3}} \tilde{w} \\
\mathcal{J}_{\xi, \mu} \eta= & -\frac{\dot{\psi}_{\xi, \mu}^{3}}{\phi_{\xi, \mu}^{3}} \frac{\partial^{2} \eta}{\left(\partial x_{0}\right)^{2}}-\frac{1}{\varepsilon}\left(\frac{\dot{\psi}_{\xi, \mu} \ddot{\psi}_{\xi, \mu}}{\phi_{\xi, \mu}^{3}}+2\left(\phi_{\xi, \mu}^{2}-\tau_{\xi, \mu}\right) \frac{\dot{\phi}_{\xi, \mu} \dot{\psi}_{\xi, \mu}}{\phi_{\xi, \mu}^{4}}\right) \frac{\partial \eta}{\partial x_{0}} \\
& -\phi_{\xi, \mu}^{-2}\left(2 \dot{\phi}_{\xi, \mu} \ddot{\psi}_{\xi, \mu}+2 \frac{\dot{\phi}_{\xi, \mu}^{2} \dot{\psi}_{\xi, \mu}}{\phi_{\xi, \mu}}+\frac{\dot{\psi}_{\xi, \mu}^{3}}{\phi_{\xi, \mu}}\right. \\
& \left.-2 \frac{\dot{\psi}_{\xi, \mu}^{2}}{\phi_{\xi, \mu}}\left(\phi_{\xi, \mu}^{2}-\tau_{\xi, \mu}\right)-2 \phi_{\xi, \mu} \dot{\phi}_{\xi, \mu}^{2}\right) R\left(\eta, X_{0}\right) X_{0} .
\end{aligned}
$$

Theorem 4.1. 1. The operator

$$
\tilde{\mathcal{L}}_{\xi, \mu}: \tilde{\Pi} C_{\varepsilon}^{2, \alpha}(S N \Gamma) \rightarrow \tilde{\Pi} C_{\varepsilon}^{0, \alpha}(S N \Gamma)
$$

is invertible and for some uniform $C$

$$
\|\tilde{w}\|_{C_{\varepsilon}^{2, \alpha}} \leq C\left\|\tilde{\mathcal{L}}_{\xi, \mu} \tilde{w}\right\|_{C_{\varepsilon}^{0, \alpha}}
$$

2. The operator

$$
\mathcal{J}_{\xi, \mu}: C_{x_{0}, \varepsilon}^{2, \alpha}(\Gamma, N \Gamma) \rightarrow C_{\varepsilon}^{\alpha}(\Gamma, N \Gamma)
$$

is invertible and for some uniform $C$

$$
\|\eta\|_{C_{x_{0}, \varepsilon}^{2, \alpha}} \leq C\left\|\mathcal{J}_{\xi, \mu} \eta\right\|_{C_{\varepsilon}^{\alpha}}
$$

3. If

$$
\mathcal{J}_{\xi, \mu} \hat{\eta}=-\frac{2}{3} \varepsilon \dot{\phi}_{\xi, \mu} P_{1}\left(R\left(\Upsilon_{\theta}, X_{0}, \Upsilon, \Upsilon_{\theta}\right)\right)
$$

we have

$$
\|\hat{\eta}\|_{C_{x_{0}, \varepsilon}^{2, \alpha}} \leq C \varepsilon^{2}
$$


Proof. For the first item, consider the bilinear functional on the space $\tilde{\Pi} W_{\varepsilon}^{1,2}(S N \Gamma)$

$$
B_{\xi, \mu}(w, v)=\int_{S N \Gamma}\left(v\left(\phi_{\xi, \mu} \tilde{\mathcal{L}}_{\xi, \mu}\right) w\right) d \theta d \psi
$$

By using exactly the same argument as used in Subsection 3.2 , we can prove the results.

For the second and third item, consider $\frac{\dot{\psi}_{\xi, \mu}^{3}}{\phi_{\xi, \mu}} \mathcal{J}_{\xi, \mu} \eta$. Let $\frac{\partial}{\partial y_{0}}=\frac{\dot{\psi}_{\xi, \mu}^{3}}{\phi_{\xi, \mu}^{2}} \frac{\partial}{\partial x_{0}}$. From Corollary 3.20, we know $y_{0}\left(L_{\Gamma}\right)=I_{1}^{\prime} L_{\Gamma}, I_{1}^{\prime}=I_{1}+O\left(\varepsilon^{2}\right)$. Now we define $\tilde{y}=$ $\left(I_{1}^{\prime}\right)^{-1} y_{0}$ and define $\mathfrak{F}$ and $P_{p}^{\mathfrak{F}(p)}$ as in the paragraph before Lemma 3.2 .

$$
\begin{aligned}
\frac{\dot{\psi}_{\xi, \mu}^{3}}{\phi_{\xi, \mu}} \mathcal{J}_{\xi, \mu} \eta(q)= & -\frac{\partial^{2} \eta}{\partial y_{0}^{2}}(q)-\left.\Psi_{1}\left(\phi_{\xi, \mu}, \frac{\partial \phi_{\xi, \mu}}{\partial \psi}\right) R\right|_{q}\left(\eta(q), X_{0}\right) X_{0}+\frac{1}{\varepsilon} \rho L(\eta) \\
= & \tilde{\mathcal{J}}_{A}^{\prime} \eta+\left(I_{2}^{\prime}-\left.\Psi_{1}\right|_{q}\right)\left(P_{\mathfrak{F}^{-1}(q)}^{q} R\right)\left(\eta(q), X_{0}\right) X_{0} \\
& +\left.\Psi_{1}\right|_{x_{0}}\left(P_{\mathfrak{F}^{-1}(q)}^{q} R-\left.R\right|_{q}\right)\left(\eta(q), X_{0}\right) X_{0}+\frac{1}{\varepsilon} \rho L(\eta) \\
= & \tilde{\mathcal{J}}_{A}^{\prime} \eta+\left(I_{2}^{\prime}-\left.\Psi_{1}\right|_{q}\right)\left(P_{\mathfrak{F}^{-1}(q)}^{q} R\right)\left(\eta(q), X_{0}\right) X_{0}+\varepsilon L(\eta),
\end{aligned}
$$

where $I_{2}^{\prime}=I_{2}+O\left(\varepsilon^{2}\right)$ and $I_{1}^{\prime 2} I_{2}^{\prime}=1 . I_{1}^{\prime 2} \tilde{\mathcal{J}}_{A}^{\prime}$ is conjugate to $\mathcal{J}_{A}$. The rest of the proof is similar to Theorem 3.3 However, the primitive of $\left(I_{2}^{\prime}-\Psi_{1}\right)$ and $\frac{\dot{\psi}_{\xi, \mu}^{3}}{\phi_{\xi, \mu}} \dot{\phi}_{\xi, \mu}$ may not be global smooth function on the geodesic. Nevertheless, from Corollary 3.13 , we know

$$
\begin{aligned}
\chi\left(y_{1}\right) & =\int_{0}^{y_{1}}\left(I_{2}^{\prime}-\Psi_{1}\right) d y_{0}=O(\varepsilon), \\
\chi\left(L_{\Gamma}\right) & =O\left(\varepsilon^{2}\right) .
\end{aligned}
$$

From the proof of Lemma 3.4 by noticing

$$
I_{2}^{\prime}-\Psi_{1}=\left(I_{2}^{\prime}-\Psi_{1}-\frac{\chi\left(L_{\Gamma}\right)}{L_{\Gamma}}\right)+\frac{\chi\left(L_{\Gamma}\right)}{L_{\Gamma}},
$$

we can prove the second item. For $\frac{\dot{\psi}_{\xi, \mu}^{3}}{\phi_{\xi, \mu}} \dot{\phi}_{\xi, \mu}$ we can do the same thing. So all the argument in Theorem 3.3 works here and we can prove this theorem.

Consider

$$
\left\{\begin{aligned}
\frac{1}{\varepsilon} \tilde{\mathcal{L}}_{\xi, \mu} \tilde{w}= & -\Pi_{0}\left(\varepsilon\left(F_{1}\left(\phi_{\xi, \mu}, \zeta_{\xi, \mu}\right) \star R_{1}+F_{2}\left(\phi_{\xi, \mu}, \zeta_{\xi, \mu}\right) \star R_{2}\right)+\left(\varepsilon^{2}+\varepsilon \rho\right) E_{\xi, \mu}\right. \\
& \left.+F_{3}\left(\phi_{\xi, \mu}, \zeta_{\xi, \mu}\right) \star R_{3}(\eta)+\left(\varepsilon+\varepsilon^{-1} \rho\right) L_{\xi, \mu}(\tilde{w}, \eta)+\varepsilon^{-1}(1+\rho) Q_{\xi, \mu}(\tilde{w}, \eta)\right), \\
\mathcal{J}_{\xi, \mu} \eta= & -P_{1}\left(\varepsilon F_{2}\left(\phi_{\xi, \mu}, \zeta_{\xi, \mu}\right) \star R_{2}\right)+\left(\varepsilon^{2}+\varepsilon \rho\right) E_{\xi, \mu}+\left(\varepsilon+\varepsilon^{-1} \rho\right) L_{\xi, \mu}(\tilde{w}, \eta) \\
& \left.+\varepsilon^{-1}(1+\rho) Q_{\xi, \mu}(\tilde{w}, \eta)\right) .
\end{aligned}\right.
$$


From Theorem 4.1 and the properties of $L_{\xi, \mu}, Q_{\xi, \mu}, E_{\xi, \mu}$ in Definition 4 we can solve this system and get $\left(\tilde{w}_{\xi, \mu}, \eta_{\xi, \mu}\right)$ which satisfies

$$
\left\|\tilde{w}_{\xi, \mu}\right\|_{C_{\varepsilon}^{2, \alpha}}+\left\|\eta_{\xi, \mu}\right\|_{C_{x_{0}, \varepsilon}^{2, \alpha}} \leq C \varepsilon^{2} .
$$

Moreover, from Theorem 3.7. Theorem 4.1. Definition 4, we can prove

\section{Lemma 4.2.}

$$
\begin{cases}\left\|\tilde{w}_{\xi+\Delta \xi, \mu+\Delta \mu}-\tilde{w}_{\xi, \mu}\right\|_{C_{\varepsilon}^{2, \alpha}} & \leq C \varepsilon^{2}\left(\varepsilon\|\Delta \mu\|_{C_{\varepsilon}^{\alpha}}+\|\Delta \xi\|_{C_{x_{0}}^{1}}\right) \\ \left\|\eta_{\xi+\Delta \xi, \mu+\Delta \mu}-\eta_{\xi, \mu}\right\|_{C_{x_{0}, \varepsilon}^{2, \alpha}} & \leq C \varepsilon\left(\varepsilon\|\Delta \mu\|_{C_{\varepsilon}^{\alpha}}+\|\Delta \xi\|_{C_{x_{0}}^{1}}\right) .\end{cases}
$$

One can calculate

$$
\begin{aligned}
& H\left(\mathcal{D}_{\phi_{\xi, \mu}, p_{0}, \varepsilon}\left(\tilde{w}_{\xi, \mu}, \eta_{\xi, \mu}\right)\right)-\frac{2}{\varepsilon}=\Pi_{0}\left(H\left(\mathcal{D}_{\phi_{\xi, \mu}, p_{0}, \varepsilon}\left(\tilde{w}_{\xi, \mu}, \eta_{\xi, \mu}\right)\right)-\frac{2}{\varepsilon}\right) \\
= & F_{4}\left(\phi_{\xi, \mu}, \zeta_{\xi, \mu}\right)\left(\Pi_{0}\left(R\left(\Upsilon, X_{0}, \eta_{\xi, \mu}, \Upsilon\right)\right)+\xi\left(x_{0}\right)\right)+\varepsilon^{2} \mu+\Pi_{0}\left(\left(\varepsilon^{2}+\varepsilon \rho\right) E_{\xi, \mu}\right. \\
& \left.+\left(\varepsilon+\varepsilon^{-1} \rho\right) L_{\xi, \mu}(\tilde{w}, \eta)+\varepsilon^{-1}(1+\rho) Q_{\xi, \mu}(\tilde{w}, \eta)\right)+\varepsilon^{2} \omega_{\xi, \mu} \phi_{\xi, \mu}^{-1} \frac{d \phi_{\xi, \mu}}{d \psi} .
\end{aligned}
$$

Now we define a map

$$
\begin{aligned}
\Omega: C_{x_{0}}^{1} \times C_{\varepsilon}^{\alpha} & \rightarrow C_{x_{0}}^{1} \times C_{\varepsilon}^{\alpha} \\
(\xi, \mu) & \mapsto\left(\Omega^{1}(\xi, \mu), \Omega^{2}(\xi, \mu)\right)
\end{aligned}
$$

where

$$
\left\{\begin{aligned}
\Omega^{1}(\xi, \mu)= & -\Pi_{0}\left(R\left(\Upsilon, X_{0}, \eta_{\xi, \mu}, \Upsilon\right)\right) \\
\Omega^{2}(\xi, \mu)= & -\varepsilon^{-2} \Pi_{0}\left(\left(\varepsilon^{2}+\varepsilon \rho\right) E_{\xi, \mu}+\left(\varepsilon+\varepsilon^{-1} \rho\right) L_{\xi, \mu}(\tilde{w}, \eta)\right. \\
& \left.+\varepsilon^{-1}(1+\rho) Q_{\xi, \mu}(\tilde{w}, \eta)\right) .
\end{aligned}\right.
$$

Lemma 4.3. For fixed $C_{1}, C_{2}$ if $\|\xi\|_{C_{x_{0}}^{1}} \leq C_{1} \varepsilon^{2},\|\mu\|_{C_{\varepsilon}^{\alpha}} \leq C_{2}$, there is $C>0$ which does not depend on $\varepsilon$ such that

$$
\begin{aligned}
& \left\|\left(\Omega^{1}\left(\xi_{1}, \mu_{1}\right), \Omega^{2}\left(\xi_{1}, \mu_{1}\right)\right)-\left(\Omega^{1}\left(\xi_{2}, \mu_{2}\right), \Omega^{2}\left(\xi_{2}, \mu_{2}\right)\right)\right\|_{\varepsilon, \alpha} \\
\leq & C \varepsilon\left\|\left(\xi_{1}-\xi_{2}, \mu_{1}-\mu_{2}\right)\right\|_{\varepsilon, \alpha}
\end{aligned}
$$

where the $\|(\cdot, \cdot)\|_{\varepsilon, \alpha}$ norm is defined in Subsection 2.1.3.

Proof.

$$
\begin{aligned}
& \left\|\left(\Omega^{1}\left(\xi_{1}, \mu_{1}\right), \Omega^{2}\left(\xi_{1}, \mu_{1}\right)\right)-\left(\Omega^{1}\left(\xi_{2}, \mu_{2}\right), \Omega^{2}\left(\xi_{2}, \mu_{2}\right)\right)\right\|_{\varepsilon, \alpha} \\
\leq & \left\|\Omega^{1}\left(\xi_{1}, \mu_{1}\right)-\Omega^{1}\left(\xi_{2}, \mu_{2}\right)\right\|_{C_{x_{0}}^{1}}+\varepsilon\left\|\Omega^{2}\left(\xi_{1}, \mu_{1}\right)-\Omega^{2}\left(\xi_{2}, \mu_{2}\right)\right\|_{C_{\varepsilon}^{\alpha}} .
\end{aligned}
$$

From Lemma 4.2 .

$$
\begin{aligned}
\left\|\Omega^{1}\left(\xi_{1}, \mu_{1}\right)-\Omega^{1}\left(\xi_{2}, \mu_{2}\right)\right\|_{C_{x_{0}}^{1}} & \leq C\left\|\eta_{\xi_{1}, \mu_{1}}-\eta_{\xi_{2}, \mu_{2}}\right\|_{C_{x_{0}}^{1}} \\
& \leq C \varepsilon\left\|\left(\xi_{1}-\xi_{2}, \mu_{1}-\mu_{2}\right)\right\|_{\varepsilon, \alpha}
\end{aligned}
$$


Notice that $\rho=\rho_{\xi, \mu}=O\left(\varepsilon^{2}\right)$. From Definition 4 and Lemma 4.2 , we have

$$
\begin{aligned}
\left\|\Omega^{2}\left(\xi_{1}, \mu_{1}\right)-\Omega^{2}\left(\xi_{2}, \mu_{2}\right)\right\|_{C_{\varepsilon}^{\alpha} \leq} & C \varepsilon^{-2}\left(\varepsilon^{2}\left|E_{\xi_{1}, \mu_{1}}-E_{\xi_{2}, \mu_{2}}\right|\right. \\
& +\varepsilon\left|L_{\xi_{1}, \mu_{1}}\left(\tilde{w}_{\xi_{1}, \mu_{1}}, \eta_{\xi_{1}, \mu_{1}}\right)-L_{\xi_{2}, \mu_{2}}\left(\tilde{w}_{\xi_{2}, \mu_{2}}, \eta_{\xi_{2}, \mu_{2}}\right)\right| \\
& +\varepsilon^{-1}\left|Q_{\xi_{1}, \mu_{1}}\left(\tilde{w}_{\xi_{1}, \mu_{1}}, \eta_{\xi_{1}, \mu_{1}}\right)-Q_{\xi_{2}, \mu_{2}}\left(\tilde{w}_{\xi_{2}, \mu_{2}}, \eta_{\xi_{2}, \mu_{2}}\right)\right| \\
& \left.+O(\varepsilon)\left|\rho_{\xi_{1}, \mu_{1}}-\rho_{\xi_{2}, \mu_{2}}\right|\right) \\
\leq & C\left\|\left(\xi_{1}-\xi_{2}, \mu_{1}-\mu_{2}\right)\right\|_{\varepsilon, \alpha} .
\end{aligned}
$$

So we proved this lemma.

If $\xi^{0}=0, \mu^{0}=0$, we can get $\tilde{w}_{0,0}, \eta_{0,0}$, whose norms only depend on the curvature terms along the geodesic. So there is $C_{7}$ which only depends on the norms of the curvatures along the geodesic, such that

$$
\begin{aligned}
\left\|\Omega^{1}(0,0)\right\|_{C_{x_{0}}^{1}} & \leq C_{7} \varepsilon^{2} \\
\varepsilon\left\|\Omega^{2}(0,0)\right\|_{C_{\varepsilon}^{\alpha}} & \leq C_{7} \varepsilon
\end{aligned}
$$

and if we assume

$$
\begin{aligned}
\xi^{1} & =\Omega^{1}(0,0) \\
\mu^{1} & =\Omega^{2}(0,0)
\end{aligned}
$$

from 54

$$
\begin{aligned}
\left\|\Omega^{1}\left(\xi^{1}, \mu^{1}\right)-\xi^{1}\right\|_{C_{x_{0}}^{1}} & \leq C_{7} \varepsilon^{2} \\
\varepsilon\left\|\Omega^{2}\left(\xi^{1}, \mu^{1}\right)-\mu^{1}\right\|_{C_{\varepsilon}^{\alpha}} & \leq C_{7} \varepsilon^{2},
\end{aligned}
$$

where we can use the same constant $C_{7}$. Then

$$
\left\|\Omega\left(\xi^{1}, \mu^{1}\right)-\left(\xi^{1}, \mu^{1}\right)\right\|_{\varepsilon, \alpha} \leq 2 C_{7} \varepsilon^{2} .
$$

Let

$$
\Xi\left(5 C_{7}\right)=\left\{(\xi, \mu):\left\|(\xi, \mu)-\left(\xi^{1}, \mu^{1}\right)\right\|_{\varepsilon, \alpha} \leq 5 C_{7} \varepsilon^{2}\right\}
$$

We assume $C_{1}=C_{2}=10 C_{7}$. Let

$$
\Xi\left(C_{1}, C_{2}\right)=\left\{(\xi, \mu):\|\xi\|_{C_{x_{0}}^{1}} \leq C_{1} \varepsilon^{2},\|\mu\|_{C_{\varepsilon}^{\alpha}} \leq C_{2}\right\} .
$$

It is obvious that

$$
\Xi\left(5 C_{7}\right) \subset \Xi\left(C_{1}, C_{2}\right) .
$$

So if $\left(\xi_{1}, \mu_{1}\right),\left(\xi_{2}, \mu_{2}\right) \in \Xi\left(5 C_{7}\right)$, then

$$
\left\|\Omega\left(\xi_{1}, \mu_{1}\right)-\Omega\left(\xi_{2}, \mu_{2}\right)\right\|_{\varepsilon, \alpha} \leq C \varepsilon\left\|\left(\xi_{1}-\xi_{2}, \mu_{1}-\mu_{2}\right)\right\|_{\varepsilon, \alpha} .
$$

If we choose $\varepsilon$ such that

$$
C \varepsilon \leq \frac{1}{100}
$$


then $\Omega_{2}$ maps $\Xi\left(5 C_{7}\right)$ into itself. Note that $\Xi\left(5 C_{7}\right)$ is a complete metric space. From fixed point theorem, there is a unique

$$
(\hat{\xi}, \hat{\mu}) \in \Xi\left(5 C_{7}\right)
$$

such that

$$
\Omega(\hat{\xi}, \hat{\mu})=(\hat{\xi}, \hat{\mu}) .
$$

For this $(\hat{\xi}, \hat{\mu})$, we have

$$
H\left(\mathcal{D}_{\phi_{\hat{\xi}, \hat{\mu}}, p_{0}, \varepsilon}\left(\tilde{w}_{\hat{\xi}, \hat{\mu}}, \eta_{\hat{\xi}, \hat{\mu}}\right)\right)=\frac{2}{\varepsilon}+\varepsilon^{2} \omega_{\hat{\xi}, \hat{\mu}} \phi_{\hat{\xi}, \hat{\mu}}^{-1} \frac{\partial \phi_{\hat{\xi}, \hat{\mu}}}{\partial \psi} .
$$

\subsection{The energy of the surface and the last step of the proof.}

So far what we've got is a global smooth surface $\mathcal{D}_{\phi_{\hat{\xi}, \hat{\mu}}, p_{0}, \varepsilon}\left(\tilde{w}_{\hat{\xi}, \hat{\mu}}, \eta_{\hat{\xi}, \hat{\mu}}\right)$ whose mean curvature is

$$
\frac{2}{\varepsilon}+\omega_{\hat{\xi}, \hat{\mu}} \varepsilon^{2} \phi_{\hat{\xi}, \hat{\mu}}^{-1} \frac{\partial \phi_{\hat{\xi}, \hat{\mu}}}{\partial \psi} .
$$

In (27), we have chosen a point $p_{0}$ where $\psi=0$ (and also $x_{0}=0$ ), i.e. $\phi(\psi)$ attains a local minimum at $p_{0}$. We call $p_{0}$ the starting point. From the analysis above, we find that $\hat{\xi}, \hat{\mu}$ and $\omega_{\hat{\xi}, \hat{\mu}}$ only depend on $p_{0}$. The last thing we can do is to move the starting point along the geodesic such that $\omega_{\hat{\xi}, \hat{\mu}}=0$. View $\psi \in\left(-\varepsilon_{0}, \varepsilon_{0}\right)$ as local coordinate about $p_{0}$ and $\psi\left(p_{0}\right)=0$. Now choose $\psi=\delta$ instead of $\psi=0$ as the starting point. We can do all the analysis above and get $\left(\hat{\xi}_{\delta}, \hat{\mu}_{\delta}, \omega_{\delta}=\omega_{\hat{\xi}_{\delta}, \hat{\mu}_{\delta}}, \phi_{\delta}(\delta)=\phi_{\hat{\xi}_{\delta}, \hat{\mu}_{\delta}}(\delta), \phi_{\delta}=\phi_{\hat{\xi}_{\delta}, \hat{\mu}_{\delta}}, \tilde{w}_{\delta}, \eta_{\delta}\right)$ which satisfy similar estimates as $\delta=0$ case and

$$
H\left(\mathcal{D}_{\phi_{\delta}, \delta, \varepsilon}\left(\tilde{w}_{\delta}, \eta_{\delta}\right)\right)=\frac{2}{\varepsilon}+\omega_{\delta} \varepsilon^{2} \phi_{\delta}^{-1} \frac{\partial \phi_{\delta}}{\partial \psi} .
$$

We make the following notations

$$
\begin{aligned}
\left.\frac{\partial f_{\delta}(\psi)}{\partial \delta}\right|_{\delta=0} & =\lim _{\delta \rightarrow 0} \frac{f_{\delta}(\psi)-f_{0}(\psi)}{\delta} \\
\left.\frac{\partial^{\prime} f_{\delta}(\psi)}{\partial \delta}\right|_{\delta=0} & =\lim _{\delta \rightarrow 0} \frac{f_{\delta}(\psi)-f_{0}(\psi-\delta)}{\delta} .
\end{aligned}
$$

We can take a new angle of view. We identify different starting points and imagine that the curvature terms are translated along the geodeisc. We know

$$
\frac{\partial^{\prime}}{\partial \delta} R_{i}=\varepsilon \bar{R}_{i}
$$

and $\bar{R}_{i}$ 's derivatives with respect to $x_{0}$ are bounded. Besides, $\bar{R}_{i}$ belongs to the same subspace (range of $\Pi_{0}, \Pi_{1}, \tilde{\Pi}$ ) as $R_{i}$ does. So if we revise each mode, we know that $\phi_{\delta}, \tilde{w}_{\delta}, \eta_{\delta}$ vary smoothly in $\delta$. We want to study $\frac{\partial^{\prime} \phi_{\delta}}{\partial \delta}, \frac{\partial^{\prime} \tilde{w}_{\delta}}{\partial \delta}, \frac{\partial^{\prime} \eta_{\delta}}{\partial \delta}$.

Now we start with the initial surface $\mathcal{D}_{\phi_{\hat{\xi}, \hat{\mu}}, p_{0}, \varepsilon}\left(\tilde{w}_{\hat{\xi}, \hat{\mu}}, \eta_{\hat{\xi}, \hat{\mu}}\right) \cdot \phi_{\hat{\xi}, \hat{\mu}}$ satisfies 27] with $\xi=\hat{\xi}, \mu=\hat{\mu}, \phi(0)=\phi(0)_{\hat{\xi}, \hat{\mu}}, \omega=\omega_{\hat{\xi}, \hat{\mu}}$. Heuristically when $\delta$ has a variation 
of size 1 , the $C_{x_{0}}^{1}$ norm of $R_{i}$ will has a variation of $O(\varepsilon)$. At first we fix $\xi=\hat{\xi}$ and $\mu=\hat{\mu}$. By analyzing both nonlinear ODE and its linearized equations, we find $\omega$ should be perturbed as large as $O(\varepsilon)$ and $\phi(0)$ should be perturbed as large as $O\left(\varepsilon^{3}\right)$ to match the boundary value. Hence we know $\left\|\phi_{\delta}(\psi+\delta)-\phi_{\hat{\xi}, \hat{\mu}}(\psi)\right\|_{C_{\varepsilon}^{2, \alpha}}=$ $O\left(\varepsilon^{2}\right)$. From Theorem 4.1, 52 and Definition 4 we have $\left\|\tilde{w}_{\delta}(\psi+\delta)-\tilde{w}(\psi)\right\|_{C_{\varepsilon}^{2, \alpha}} \leq$ $C \varepsilon^{3},\left\|\eta_{\delta}(\psi+\delta)-\eta(\psi)\right\|_{C_{x_{0}, \varepsilon}^{2, \alpha}} \leq C \varepsilon^{3}$. Now we replace $(\hat{\xi}, \hat{\mu})$ with $\left(\Omega^{1}(\hat{\xi}, \hat{\mu}), \Omega^{2}(\hat{\xi}, \hat{\mu})\right)$. From $(53), \hat{\xi}$ has a $O\left(\varepsilon^{3}\right)$ variation and $\hat{\mu}$ has a variation of $O(\varepsilon)$ which implies an $\varepsilon^{2}$ variation again on $\phi_{\delta}$ by 29 and hence a $O\left(\varepsilon^{4}\right)$ variation on $\tilde{w}_{\delta}$ and a $O\left(\varepsilon^{3}\right)$ variation on $\eta_{\delta}$. However, in the second step of iteration $\hat{\mu}$ only has a variation of $O\left(\varepsilon^{2}\right)$. So the iteration argument works.

\begin{tabular}{|c|c|c|c|c|c|c|}
\hline & 1 & 2 & 3 & $\cdots$ & $\mathrm{k}$ & $\cdots$ \\
\hline $\begin{array}{c}\omega_{\delta} \\
|\cdot|\end{array}$ & $\varepsilon$ & $\varepsilon$ & $\varepsilon^{2}$ & $\cdots$ & $\varepsilon^{k-1}$ & $\cdots$ \\
\hline $\begin{array}{c}\phi_{\delta}(\delta) \\
|\cdot|\end{array}$ & $\varepsilon^{3}$ & $\varepsilon^{3}$ & $\varepsilon^{4}$ & $\cdots$ & $\varepsilon^{k+1}$ & $\cdots$ \\
\hline $\begin{array}{c}\phi_{\delta}(\cdot+\delta) \\
\|\cdot\|_{C_{\varepsilon}^{2, \alpha}}\end{array}$ & $\varepsilon^{2}$ & $\varepsilon^{2}$ & $\varepsilon^{3}$ & $\ldots$ & $\varepsilon^{k}$ & $\cdots$ \\
\hline $\begin{array}{c}\tilde{w}_{\delta}(\cdot+\delta) \\
\|\cdot\|_{C_{\varepsilon}^{2, \alpha}}\end{array}$ & $\varepsilon^{3}$ & $\varepsilon^{4}$ & $\varepsilon^{5}$ & $\cdots$ & $\varepsilon^{k+2}$ & $\cdots$ \\
\hline $\begin{array}{c}\eta_{\delta}(\cdot+\delta) \\
\|\cdot\|_{C_{x_{0}, \varepsilon}^{2, \alpha}}\end{array}$ & $\varepsilon^{3}$ & $\varepsilon^{3}$ & $\varepsilon^{4}$ & $\cdots$ & $\varepsilon^{k+1}$ & $\cdots$ \\
\hline $\begin{array}{c}\xi_{\delta}(\cdot+\delta) \\
\|\cdot\|_{C_{x_{0}}^{1}}\end{array}$ & $\varepsilon^{3}$ & $\varepsilon^{3}$ & $\varepsilon^{4}$ & $\cdots$ & $\varepsilon^{k+1}$ & $\cdots$ \\
\hline $\begin{array}{c}\mu_{\delta}(\cdot+\delta) \\
\|\cdot\|_{C_{\varepsilon}^{\alpha}}\end{array}$ & $\varepsilon$ & $\varepsilon^{2}$ & $\varepsilon^{3}$ & $\cdots$ & $\varepsilon^{k}$ & $\cdots$ \\
\hline
\end{tabular}

In the above form, for example, the $\varepsilon^{k+1}$ in $\left(\eta_{\delta}(\cdot+\delta), k\right)$ position means in the $k$ th step of iteration, $\eta_{\delta}(\cdot+\delta)$ has a variation of $\varepsilon^{k+1}$ measured in $\|\cdot\|_{C_{x_{0}, \varepsilon}^{2, \alpha}}$ norm.

The above argument can be made precise by taking $\frac{\partial^{\prime}}{\partial \delta}$ derivative to each mode as well as the expression (53).

At last we get

$$
\begin{gathered}
\left\|\left.\frac{\partial^{\prime}}{\partial \delta} \phi_{\delta}\right|_{\delta=0}\right\|_{C_{\varepsilon}^{2, \alpha}} \leq C \varepsilon^{2}, \\
\left\|\left.\frac{\partial^{\prime}}{\partial \delta} \eta_{\delta}\right|_{\delta=0}\right\|_{C_{x_{0}, \varepsilon}^{2, \alpha}} \leq C \varepsilon^{3}, \\
\left\|\left.\frac{\partial^{\prime}}{\partial \delta} \tilde{w}_{\delta}\right|_{\delta=0}\right\|_{C_{\varepsilon}^{2, \alpha}} \leq C \varepsilon^{3} .
\end{gathered}
$$

So we have 


$$
\begin{aligned}
& \left.\frac{\partial}{\partial \delta}\left(\varepsilon\left(\phi_{\delta}+\tilde{w}_{\delta}\right)+<\eta_{\delta}, \Upsilon>\right)\right|_{\delta=0} \\
= & \left.\frac{\partial^{\prime}}{\partial \delta}\left(\varepsilon\left(\phi_{\delta}+\tilde{w}_{\delta}\right)+<\eta_{\delta}, \Upsilon>\right)\right|_{\delta=0}-\left.\frac{\partial}{\partial \psi}\left(\varepsilon\left(\phi_{\hat{\xi}, \hat{\mu}}+\tilde{w}_{\hat{\xi}, \hat{\mu}}\right)+<\eta_{\hat{\xi}, \hat{\mu}}, \Upsilon\right)\right|_{\psi=0} \\
= & -\varepsilon \frac{\partial \phi_{\hat{\xi}, \hat{\mu}}}{\partial \psi}+O\left(\varepsilon^{3}\right) .
\end{aligned}
$$

Consider the energy functional of the surface $\Sigma_{\delta}=\mathcal{D}_{\phi_{\delta}, \delta, \varepsilon}\left(\tilde{w}_{\delta}, \eta_{\delta}\right)$

$$
E N\left(\Sigma_{\delta}\right)=\operatorname{Area}\left(\Sigma_{\delta}\right)-\frac{2}{\varepsilon} \operatorname{Vol}\left(\Sigma_{\delta}\right)
$$

We have

$$
\begin{aligned}
& \left.\frac{d}{d \delta} \operatorname{EN}\left(\Sigma_{\delta}\right)\right|_{\delta=0} \\
= & \frac{d}{d \delta}\left(\operatorname{Area}\left(\Sigma_{\delta}\right)-\frac{2}{\varepsilon} \operatorname{Vol}\left(\Sigma_{\delta}\right)\right) \\
= & \left.\int_{\Sigma_{0}} H \frac{\partial}{\partial \delta}\left(\varepsilon\left(\phi_{\delta}(\psi)+\tilde{w}_{\delta}\right)+<\eta_{\delta}, \Upsilon>\right)\right|_{\delta=0}<N, \Upsilon>d S \\
& -\left.\frac{2}{\varepsilon} \int_{\Sigma_{0}} \frac{\partial}{\partial \delta}\left(\varepsilon\left(\phi_{\delta}(\psi)+\tilde{w}_{\delta}\right)+<\eta_{\delta}, \Upsilon>\right)\right|_{\delta=0}<N, \Upsilon>d S \\
= & -\varepsilon^{3} \omega_{\hat{\xi}, \hat{\mu}} \int_{\Sigma_{0}} \phi_{\hat{\xi}, \hat{\mu}}^{-1}\left[\left(\frac{\partial \phi_{\hat{\xi}, \hat{\mu}}}{\partial \psi}\right)^{2}+O\left(\varepsilon^{2}\right)\right]<N, \Upsilon>d S,
\end{aligned}
$$

where $\Sigma_{0}=\mathcal{D}_{\phi_{\hat{\xi}, \hat{\mu}}, p_{0}, \varepsilon}\left(\tilde{w}_{\hat{\xi}, \hat{\mu}}, \eta_{\hat{\xi}, \hat{\mu}}\right)$. Note that

$$
\int_{\Sigma_{0}} \phi_{\hat{\xi}, \hat{\mu}}^{-1}\left(\frac{\partial \phi_{\hat{\xi}, \hat{\mu}}}{\partial \psi}\right)^{2}<N, \Upsilon>d S
$$

is always positive.

Similarly for $\delta \in\left[0, \frac{L_{\Gamma}}{\varepsilon}\right]$, we can prove that

$$
\begin{aligned}
& \left.\frac{d}{d \delta} \operatorname{EN}\left(\Sigma_{\delta}\right)\right|_{\delta=0} \\
= & -\varepsilon^{3} \omega_{\delta} \int_{\Sigma_{\delta}} \phi_{\delta}^{-1}\left[\left(\frac{\partial}{\partial \psi} \phi_{\delta}\right)^{2}+O\left(\varepsilon^{2}\right)\right]<N, \Upsilon>d S
\end{aligned}
$$

with the integral being always positive. If $E$ is constant when $\delta \in\left[0, \frac{L_{\Gamma}}{\varepsilon}\right]$, we have for every $\delta, \omega_{\delta}=0$. Then we have infinitely many Delaunay type constant mean curvature surfaces. If $E$ is not always constant, we will at least get two zeros of $\frac{d}{d \delta} E\left(\Sigma_{\delta}\right)$, where we have $\omega_{\delta}=0$. Then we get two Delaunay type constant mean curvature surfaces. The two surfaces are not the same, because they correspond to the maximal value and minimal value of $E$. That the Delaunay type CMC surfaces are embedded is evident. First the CMC surfaces constructed can be 
viewed as a smooth map from $T^{2}$ to $M$, because the unit normal bundle of $\Gamma$ has $T^{2}$ topology. And because $T^{2}$ is compact topological space, we need only to prove that this map is injective. This is easily seen from the fact that $\Gamma$ is simply closed and embedded and the estimates of the functions in each mode. So we proved the main theorem.

For Corollary 1.2, we see that the non-degeneracy condition of the Jacobi operator of the geodesic is only used in 1st mode. However, when the metric around $\Gamma$ has rotational symmetry, i.e. $\frac{\partial}{\partial \theta}$ is killing vector field, if we look at the expression of mean curvature (13), we will find that $R_{1}, R_{2}$ and $E$ have no 1st mode or high mode projections. So we may assume $\eta=0$ and $\tilde{w}=0$. The only thing that we need to do is to solve the 0th mode. So we can use the procedure in Subsection 3.5 to solve 0th mode up to the kernel $\frac{\partial \phi}{\partial \psi}$. And then we use the same argument as in this Subsection to remove the kernel. So we can get the CMC surfaces of Delaunay type.

\section{A The calculation of the mean curvature}

We prove 13 here. By definition we have

$$
\begin{aligned}
H\left(\mathcal{D}_{\phi_{\tau_{0}}, p_{0}, \varepsilon}(w, \eta)\right)= & g^{s s}<N, \nabla_{\partial_{s}} \partial_{s}>+2 g^{s \theta}<N, \nabla_{\partial_{\theta}} \partial_{s}>+g^{\theta \theta}<N, \nabla_{\partial_{\theta}} \partial_{\theta}> \\
= & \frac{1}{k}\left(g^{s s}<k N, \nabla_{\partial_{s}} \partial_{s}>+2 g^{s \theta}<k N, \nabla_{\partial_{\theta}} \partial_{s}>\right. \\
& \left.+g^{\theta \theta}<k N, \nabla_{\partial_{\theta}} \partial_{\theta}>\right) .
\end{aligned}
$$

First, we would like to point out that in the following, terms like $\eta, \frac{\partial \eta}{\partial x_{0}}$ and $\varepsilon \frac{\partial^{2} \eta}{\partial x_{0}^{2}}$ are collected in $L(w, \eta)$. It is also similar for $Q(w, \eta)$. This is why in Definition 2 . we use $C_{x_{0}, \varepsilon}^{2, \alpha}$ norm of $\eta$.

$$
\begin{aligned}
g^{s s}< & k N, \nabla_{\partial_{s}} \partial_{s}>=g^{s s}<N_{0}+a_{1} \partial_{s}+a_{2} \partial_{\theta}, \nabla_{\partial_{s}} \partial_{s}> \\
<\partial_{s}, \nabla_{\partial_{s}} \partial_{s}>= & \frac{1}{2} \partial_{s}<\partial_{s}, \partial_{s}> \\
= & \frac{\varepsilon^{2}}{2} \partial_{s}\left(\phi^{2}+\varepsilon^{2} \phi^{2} \dot{\psi}^{2} R\left(\Upsilon, X_{0}, \Upsilon, X_{0}\right)+2 \varepsilon \phi \dot{\psi}^{2} R\left(\Upsilon, X_{0}, \eta, X_{0}\right)\right. \\
& +\frac{4}{3} \varepsilon \phi \dot{\phi} \dot{\psi} R\left(\Upsilon, X_{0}, \eta, \Upsilon\right)+2 \dot{\phi} \frac{\partial w}{\partial s}+2 \dot{\phi} \dot{\psi}<\Upsilon, \frac{\partial \eta}{\partial x_{0}}>_{e} \\
& \left.+\varepsilon^{2} L(w, \eta)+Q(w, \eta)+O\left(\varepsilon^{3}\right)\right), \\
= & \frac{\varepsilon^{2}}{2}\left(2 \phi \dot{\phi}+L(w, \eta)+Q(w, \eta)+O\left(\varepsilon^{2}\right)\right),
\end{aligned}
$$




$$
\begin{aligned}
&<\partial_{\theta}, \nabla_{\partial_{s}} \partial_{s}>= \partial_{s}<\partial_{\theta}, \partial_{s}>-<\nabla_{\partial_{s}} \partial_{\theta}, \partial_{s}> \\
&= \partial_{s}<\partial_{\theta}, \partial_{s}>-\frac{1}{2} \partial_{\theta}<\partial_{s}, \partial_{s}> \\
&= \varepsilon^{2} \partial_{s}\left(\frac{2}{3} \varepsilon^{2} \phi^{3} \dot{\psi} R\left(\Upsilon, X_{0}, \Upsilon, \Upsilon_{\theta}\right)+\frac{2}{3} \varepsilon \phi^{2} \dot{\psi}\left(R\left(\Upsilon, X_{0}, \eta, \Upsilon_{\theta}\right)\right.\right. \\
&\left.+R\left(\eta, X_{0}, \Upsilon, \Upsilon_{\theta}\right)\right)+\frac{1}{3} \varepsilon \phi^{2} \dot{\phi} R\left(\eta, \Upsilon, \Upsilon, \Upsilon_{\theta}\right)+\dot{\phi} \frac{\partial w}{\partial \theta} \\
&\left.+\phi \dot{\psi}<\frac{\partial \eta}{\partial x_{0}}, \Upsilon_{\theta}>{ }_{e}+\varepsilon^{2} L(w, \eta)+Q(w, \eta)+O\left(\varepsilon^{3}\right)\right) \\
&-\frac{\varepsilon^{2}}{2} \partial_{\theta}\left(\phi^{2}+\varepsilon^{2} \phi^{2} \dot{\psi}^{2} R\left(\Upsilon, X_{0}, \Upsilon, X_{0}\right)+2 \varepsilon \phi \dot{\psi}^{2} R\left(\Upsilon, X_{0}, \eta, X_{0}\right)\right. \\
&+\frac{4}{3} \varepsilon \phi \dot{\phi} \dot{\psi} R\left(\Upsilon, X_{0}, \eta, \Upsilon\right)+2 \dot{\phi} \frac{\partial w}{\partial s}+2 \dot{\phi} \dot{\psi}<\Upsilon, \frac{\partial \eta}{\partial x^{0}}>_{e} \\
&\left.+\varepsilon^{2} L(w, \eta)+Q(w, \eta)+O\left(\varepsilon^{3}\right)\right), \\
&= \varepsilon^{2}\left(O\left(\varepsilon^{2}\right)+L(w, \eta)+Q(w, \eta)\right) . \\
& \nabla_{\partial_{s}} \partial_{s}=\quad \nabla_{\partial_{s}}\left(\dot{\psi} X_{0}+\left(\dot{\phi}+\frac{\partial w}{\partial s}\right) \Upsilon+\dot{\psi} \frac{\partial \eta^{i}}{\partial x_{0}} X_{i}\right) \\
& \varepsilon\left(\ddot{\psi} X_{0}+\left(\ddot{\phi}+\frac{\partial^{2} w}{\partial s^{2}}\right) \Upsilon+\left(\ddot{\psi} \frac{\partial \eta^{i}}{\partial x^{0}}+\dot{\psi}^{2} \varepsilon \frac{\partial^{2} \eta^{i}}{\partial x_{0}^{2}}\right) X_{i}\right) \\
&+\varepsilon^{2}\left(\dot{\psi}^{2} \nabla_{X_{0}} X_{0}+\left(\dot{\phi}+\frac{\partial w}{\partial s}\right)^{2} \nabla \Upsilon \Upsilon+\dot{\psi}^{2}\left(\frac{\partial \eta^{i}}{\partial x_{0}}\right)\left(\frac{\partial \eta^{j}}{\partial x_{0}}\right) \nabla_{X_{i}} X_{j}\right. \\
&\left.+2 \dot{\psi}\left(\dot{\phi}+\frac{\partial w}{\partial s}\right) \nabla_{X_{0}} \Upsilon+2 \dot{\psi}^{2} \frac{\partial \eta^{i}}{\partial x_{0}} \nabla_{X_{0}} X_{i}+2\left(\dot{\phi}+\frac{\partial w}{\partial s}\right) \dot{\psi} \frac{\partial \eta^{i}}{\partial x_{0}} \nabla_{\Upsilon} X_{i}\right)
\end{aligned}
$$

We calculate

$$
<N_{0}, \nabla_{\partial_{s}} \partial_{s}>=<\frac{1}{\phi}\left(\dot{\phi} X_{0}-\dot{\psi} \Upsilon\right), \nabla_{\partial_{s}} \partial_{s}>
$$

term by term. There would be 20 terms totally.

$$
\begin{aligned}
<\frac{\dot{\phi}}{\phi} X_{0}, \varepsilon \ddot{\psi} X_{0}>= & \varepsilon \frac{\dot{\phi} \ddot{\psi}}{\phi}<X_{0}, X_{0}> \\
= & \varepsilon \frac{\dot{\phi} \ddot{\psi}}{\phi}\left(1+\varepsilon^{2} \phi^{2} R\left(\Upsilon, X_{0}, \Upsilon, X_{0}\right)_{p}+2 \varepsilon \phi R\left(\Upsilon, X_{0}, \eta, X_{0}\right)_{p}\right. \\
& \left.+\varepsilon^{2} L(w, \eta)+Q(w, \eta)+O\left(\varepsilon^{3}\right)\right) \\
= & \varepsilon \frac{\dot{\phi} \ddot{\psi}}{\phi}+\varepsilon^{3} \phi \dot{\phi} \ddot{\psi} R\left(\Upsilon, X_{0}, \Upsilon, X_{0}\right)+2 \varepsilon^{2} \dot{\phi} \ddot{\psi} R\left(\Upsilon, X_{0}, \eta, X_{0}\right) \\
& +\varepsilon^{3} L(w, \eta)+\varepsilon Q(w, \eta)+O\left(\varepsilon^{4}\right)
\end{aligned}
$$




$$
\begin{aligned}
& <\frac{\dot{\phi}}{\phi} X_{0}, \varepsilon\left(\ddot{\phi}+\frac{\partial^{2} w}{\partial s^{2}}\right) \Upsilon>=\varepsilon \frac{\dot{\phi}}{\phi}\left(\ddot{\phi}+\frac{\partial^{2} w}{\partial s^{2}}\right)<X_{0}, \Upsilon> \\
& =\varepsilon \frac{\dot{\phi}}{\phi}\left(\ddot{\phi}+\frac{\partial^{2} w}{\partial s^{2}}\right)\left(\frac{2}{3} \varepsilon \phi R\left(\Upsilon, X_{0}, \eta, \Upsilon\right)\right. \\
& \left.+\varepsilon^{2} L(w, \eta)+Q(w, \eta)+O\left(\varepsilon^{3}\right)\right) \\
& =\frac{2}{3} \varepsilon^{2} \dot{\phi} \ddot{\phi} R\left(\Upsilon, X_{0}, \eta, \Upsilon\right)+\varepsilon^{3} L(w, \eta)+\varepsilon Q(w, \eta)+O\left(\varepsilon^{4}\right), \\
& <\frac{\dot{\phi}}{\phi} X_{0}, \varepsilon \ddot{\psi} \frac{\partial \eta^{i}}{\partial x^{0}} X_{i}>=\varepsilon \frac{\dot{\phi} \ddot{\psi}}{\phi} \frac{\partial \eta^{i}}{\partial x^{0}}<X_{0}, X_{i}> \\
& =\varepsilon \frac{\dot{\phi} \ddot{\psi}}{\phi} \frac{\partial \eta^{i}}{\partial x_{0}}\left(O\left(\varepsilon^{2}\right)+\varepsilon L(w, \eta)+Q(w, \eta)+O(\varepsilon)\right) \\
& =\varepsilon^{3} L(w, \eta)+\varepsilon Q(w, \eta), \\
& <\frac{\dot{\phi}}{\phi} X_{0}, \varepsilon^{2} \dot{\psi}^{2} \frac{\partial^{2} \eta^{i}}{\partial x_{0}^{2}} X_{i}>=\varepsilon^{2} \frac{\dot{\phi} \dot{\psi}^{2}}{\phi} \frac{\partial^{2} \eta^{i}}{\partial x_{0}^{2}}\left(O\left(\varepsilon^{2}\right)+\varepsilon L(w, \eta)\right. \\
& +Q(w, \eta)+O(\varepsilon)) \\
& =\varepsilon^{3} L(w, \eta)+\varepsilon Q(w, \eta), \\
& <\frac{\dot{\phi}}{\phi} X_{0}, \varepsilon^{2} \dot{\psi}^{2} \nabla_{X_{0}} X_{0}>=\varepsilon^{2} \frac{\dot{\phi} \dot{\psi}^{2}}{\phi}<X_{0}, \nabla_{X_{0}} X_{0}> \\
& =\varepsilon^{2} \frac{\dot{\phi} \dot{\psi}^{2}}{\phi}\left(O\left(\varepsilon^{2}\right)+\varepsilon L(w, \eta)+Q(w, \eta)\right) \\
& =O\left(\varepsilon^{4}\right)+\varepsilon^{3} L(w, \eta)+\varepsilon^{2} Q(w, \eta), \\
& <\frac{\dot{\phi}}{\phi} X_{0}, \varepsilon^{2}\left(\dot{\phi}+\frac{\partial w}{\partial s}\right)^{2} \nabla_{\Upsilon} \Upsilon>=\varepsilon^{2} \frac{\dot{\phi}}{\phi}\left(\dot{\phi}+\frac{\partial w}{\partial s}\right)^{2}<X_{0}, \nabla_{\Upsilon} \Upsilon> \\
& =\varepsilon^{2} \frac{\dot{\phi}}{\phi}\left(\dot{\phi}+\frac{\partial w}{\partial s}\right)^{2}\left(\Upsilon<X_{0}, \Upsilon>-<\nabla_{X_{0}} \Upsilon, \Upsilon>\right) .
\end{aligned}
$$

The method to calculate this will be used several times, so we write it in detail. Although $\Upsilon$ actually depends on $\theta$, here we are only interested in $\nabla_{\Upsilon} \Upsilon$, we may pretend $\Upsilon$ is constant vector in the coordinates $\left\{x_{0}, x_{1}, x_{2}\right\}$. We may make such assumption where it is convenient. Evidently $\Upsilon\left(x_{k}\right)=\Upsilon^{k}$. So

$$
\begin{aligned}
\Upsilon<X_{0}, \Upsilon>= & \Upsilon\left(\frac{2}{3} R\left(X_{k}, X_{0}, X_{l}, \Upsilon\right)_{p} x_{k} x_{l}+O\left(r^{3}\right)\right) \\
= & \frac{2}{3} R\left(X_{k}, X_{0}, X_{l}, \Upsilon\right)_{p}\left(\Upsilon^{k}\left(\varepsilon(\phi+w) \Upsilon^{l}+\eta^{l}\right)\right. \\
& \left.+\left(\varepsilon(\phi+w) \Upsilon^{k}+\eta^{k}\right) \Upsilon^{l}\right)+O\left(\varepsilon^{2}\right)+\varepsilon L(w, \eta)+Q(w, \eta) \\
= & \frac{2}{3} R\left(\Upsilon, X_{0}, \eta, \Upsilon\right)+O\left(\varepsilon^{2}\right)+\varepsilon L(w, \eta)+Q(w, \eta),
\end{aligned}
$$




$$
<\nabla_{X_{0}} \Upsilon, \Upsilon>_{p}=0
$$

for all $p \in \Gamma$. We consider

$$
X_{j}<\nabla_{X_{0}} \Upsilon, \Upsilon>_{p}=<\nabla_{X_{j}} \nabla_{X_{0}} \Upsilon, \Upsilon>_{p}+<\nabla_{X_{0}} \Upsilon, \nabla_{X_{j}} \Upsilon>_{p} .
$$

From Lemma 2.1 we know $\left(\nabla_{X_{\alpha}} X_{\beta}\right)_{p}=0$. So

$$
\begin{aligned}
X_{j}<\nabla_{X_{0}} \Upsilon, \Upsilon>_{p}= & <\nabla_{X_{j}} \nabla_{X_{0}} \Upsilon, \Upsilon>_{p} \\
= & <\nabla_{X_{0}} \nabla_{X_{j}} \Upsilon, \Upsilon>_{p}+R\left(X_{j}, X_{0}, \Upsilon, \Upsilon\right)_{p} .
\end{aligned}
$$

We know $R\left(X_{j}, X_{0}, \Upsilon, \Upsilon\right)_{p}=0$. And we know $\nabla_{X_{j}} \Upsilon=0$ always holds on the geodesic. So $\left(\nabla_{X_{0}} \nabla_{X_{j}} \Upsilon\right)_{p}=0$. So $X_{j}<\nabla_{X_{0}} \Upsilon, \Upsilon>{ }_{p}=0$. And we know

$$
<\nabla_{X_{0}} \Upsilon, \Upsilon>\left(x_{0}, x_{1}, x_{2}\right)=O\left(r^{2}\right)=O\left(\varepsilon^{2}\right)+\varepsilon L(w, \eta)+Q(w, \eta) .
$$

Now we get

$$
\begin{aligned}
<\frac{\dot{\phi}}{\phi} X_{0}, \varepsilon^{2}\left(\dot{\phi}+\frac{\partial w}{\partial s}\right)^{2} \nabla \Upsilon \Upsilon>= & \frac{2}{3} \varepsilon^{2} \frac{\dot{\phi}^{3}}{\phi} R\left(\Upsilon, X_{0}, \eta, \Upsilon\right) \\
& +O\left(\varepsilon^{4}\right)+\varepsilon^{3} L(w, \eta)+\varepsilon^{2} Q(w, \eta),
\end{aligned}
$$

By using the skill above we can calculate all the remaining terms. We state the result directly.

$$
\begin{aligned}
& \varepsilon^{2}<\frac{\dot{\phi}}{\phi} X_{0}, \dot{\psi}^{2}\left(\frac{\partial \eta^{i}}{\partial x_{0}}\right)\left(\frac{\partial \eta^{j}}{\partial x_{0}}\right) \nabla_{X_{i}} X_{j}>=\varepsilon^{3} Q(w, \eta), \\
& \varepsilon^{2}<\frac{\dot{\phi}}{\phi} X_{0}, 2 \dot{\psi}\left(\dot{\phi}+\frac{\partial w}{\partial s}\right) \nabla_{X_{0}} \Upsilon>=2 \varepsilon^{3} \dot{\phi}^{2} \dot{\psi} R\left(\Upsilon, X_{0}, \Upsilon, X_{0}\right) \\
& +2 \varepsilon^{2} \frac{\dot{\phi}^{2} \dot{\psi}}{\phi} R\left(\Upsilon, X_{0}, \eta, X_{0}\right) \\
& +O\left(\varepsilon^{4}\right)+\varepsilon^{3} L(w, \eta)+\varepsilon^{2} Q(w, \eta), \\
& \varepsilon^{2}<\frac{\dot{\phi}}{\phi} X_{0}, 2 \dot{\psi}^{2} \frac{\partial \eta^{i}}{\partial x_{0}} \nabla_{X_{0}} X_{i}>=\varepsilon^{3} L(w, \eta) \\
& \varepsilon^{2}<\frac{\dot{\phi}}{\phi} X_{0}, 2\left(\dot{\phi}+\frac{\partial w}{\partial s}\right) \dot{\psi} \frac{\partial \eta^{i}}{\partial x_{0}} \nabla_{\Upsilon} X_{i}>=\varepsilon^{3} L(w, \eta)+\varepsilon^{2} Q(w, \eta), \\
& <-\frac{\dot{\psi}}{\phi} \Upsilon, \varepsilon \ddot{\psi} X_{0}>=-\varepsilon \frac{\dot{\psi} \ddot{\psi}}{\phi}<\Upsilon, X_{0}> \\
& =-\frac{2}{3} \varepsilon^{2} \dot{\psi} \ddot{\psi} R\left(\Upsilon, X_{0}, \eta, \Upsilon\right)+\varepsilon^{3} L(w, \eta)+\varepsilon Q(w, \eta)+O\left(\varepsilon^{4}\right),
\end{aligned}
$$




$$
\begin{aligned}
& <-\frac{\dot{\psi}}{\phi} \Upsilon, \varepsilon\left(\ddot{\phi}+\frac{\partial^{2} w}{\partial s^{2}}\right) \Upsilon>=-\varepsilon \frac{\ddot{\phi} \dot{\psi}}{\phi}-\varepsilon \frac{\dot{\psi}}{\phi} \frac{\partial^{2} w}{\partial s^{2}} \\
& +\varepsilon^{3} L(w, \eta)+\varepsilon Q(w, \eta)+O\left(\varepsilon^{4}\right), \\
& <-\frac{\dot{\psi}}{\phi} \Upsilon, \varepsilon \ddot{\psi} \frac{\partial \eta^{i}}{\partial x_{0}} X_{i}>=-\varepsilon \frac{\dot{\psi} \ddot{\psi}}{\phi}<\frac{\partial \eta}{\partial x_{0}}, \Upsilon> \\
& <-\frac{\dot{\psi}}{\phi} \Upsilon, \varepsilon^{2} \dot{\psi}^{2} \frac{\partial^{2} \eta^{i}}{\partial x_{0}^{2}} X_{i}>=-\varepsilon^{2} \frac{\dot{\psi}^{3}}{\phi}<\frac{\partial^{2} \eta}{\partial x_{0}^{2}}, \Upsilon> \\
& <-\frac{\dot{\psi}}{\phi} \Upsilon, \varepsilon^{2} \dot{\psi}^{2} \nabla_{X_{0}} X_{0}>=\varepsilon^{3} \dot{\psi}^{3} R\left(\Upsilon, X_{0}, \Upsilon, X_{0}\right)+\varepsilon^{2} \frac{\dot{\psi}^{3}}{\phi} R\left(\Upsilon, X_{0}, \eta, X_{0}\right) \\
& +\varepsilon^{3} L(w, \eta)+\varepsilon^{2} Q(w, \eta)+O\left(\varepsilon^{4}\right), \\
& <-\frac{\dot{\psi}}{\phi} \Upsilon, \varepsilon^{2}\left(\dot{\phi}+\frac{\partial w}{\partial s}\right)^{2} \nabla_{\Upsilon}>=\varepsilon^{3} L(w, \eta)+\varepsilon^{2} Q(w, \eta)+O\left(\varepsilon^{4}\right), \\
& <-\frac{\dot{\psi}}{\phi} \Upsilon, \varepsilon^{2} \dot{\psi}^{2}\left(\frac{\partial \eta^{i}}{\partial x_{0}}\right)\left(\frac{\partial \eta^{j}}{\partial x_{0}}\right) \nabla_{X_{i}} X_{j}>=\varepsilon^{3} Q(w, \eta), \\
& <-\frac{\dot{\psi}}{\phi} \Upsilon, \varepsilon^{2} 2 \dot{\psi}\left(\dot{\phi}+\frac{\partial w}{\partial s}\right) \nabla_{X_{0}} \Upsilon>=\varepsilon^{3} L(w, \eta)+\varepsilon^{2} Q(w, \eta)+O\left(\varepsilon^{4}\right), \\
& <-\frac{\dot{\psi}}{\phi} \Upsilon, \varepsilon^{2} 2 \dot{\psi}^{2} \frac{\partial \eta^{i}}{\partial x_{0}} \nabla_{X_{0}} X_{i}>=\varepsilon^{3} L(w, \eta), \\
& <-\frac{\dot{\psi}}{\phi} \Upsilon, \varepsilon^{2} 2\left(\dot{\phi}+\frac{\partial w}{\partial s}\right) \dot{\psi} \frac{\partial \eta^{i}}{\partial x_{0}} \nabla_{\Upsilon} X_{i}>=\varepsilon^{3} L(w, \eta)+\varepsilon^{2} Q(w, \eta) .
\end{aligned}
$$

Collecting all the terms above and notice that

$$
\frac{\dot{\phi} \ddot{\psi}}{\phi}-\frac{\ddot{\phi} \dot{\psi}}{\phi}=\phi^{2}-\tau_{0}
$$

we get

$$
\begin{aligned}
<N_{0}, \nabla_{\partial_{s}} \partial_{s}>= & \varepsilon\left(\phi^{2}-\tau_{0}\right)-\varepsilon \frac{\dot{\psi}}{\phi} \frac{\partial^{2} w}{\partial s^{2}}-\varepsilon^{2} \frac{\dot{\psi}^{3}}{\phi}<\frac{\partial^{2} \eta}{\left(\partial x_{0}\right)^{2}}, \Upsilon>-\varepsilon \frac{\dot{\psi} \ddot{\psi}}{\phi}<\frac{\partial \eta}{\partial x_{0}}, \Upsilon> \\
& +\varepsilon^{3}\left(\phi \dot{\phi} \ddot{\psi}+2 \dot{\phi}^{2} \dot{\psi}+\dot{\psi}^{3}\right) R\left(\Upsilon, X_{0}, \Upsilon, X_{0}\right) \\
& +\varepsilon^{2}\left(2 \dot{\phi} \ddot{\psi}+2 \frac{\dot{\phi}^{2} \dot{\psi}}{\phi}+\frac{\dot{\psi}^{3}}{\phi}\right) R\left(\Upsilon, X_{0}, \eta, X_{0}\right) \\
& +\varepsilon^{2}\left(\frac{2}{3} \dot{\phi} \ddot{\phi}+\frac{2}{3} \frac{\dot{\phi}^{3}}{\phi}-\frac{2}{3} \dot{\psi} \ddot{\psi}\right) R\left(\Upsilon, X_{0}, \eta, \Upsilon\right) \\
& +\varepsilon^{3} L(w, \eta)+\varepsilon Q(w, \eta)+O\left(\varepsilon^{4}\right),
\end{aligned}
$$




$$
\begin{aligned}
& g^{\theta \theta}<k N, \nabla_{\partial_{\theta}} \partial_{\theta}>=g^{\theta \theta}<N_{0}+a_{1} \partial_{s}+a_{2} \partial_{\theta}, \nabla_{\partial_{\theta}} \partial_{\theta}>. \\
& <\partial_{s}, \nabla_{\partial_{\theta}} \partial_{\theta}>=\partial_{\theta}<\partial_{s}, \partial_{\theta}>-\frac{1}{2} \partial_{s}<\partial_{\theta}, \partial_{\theta}> \\
& =\varepsilon^{2} \partial_{\theta}\left(\frac{2}{3} \varepsilon^{2} \phi^{3} \dot{\psi} R\left(\Upsilon, X_{0}, \Upsilon, \Upsilon_{\theta}\right)+\frac{2}{3} \varepsilon \phi^{2} \dot{\psi}\left(R\left(\Upsilon, X_{0}, \eta, \Upsilon_{\theta}\right)\right.\right. \\
& \left.+R\left(\eta, X_{0}, \Upsilon, \Upsilon_{\theta}\right)\right)+\frac{1}{3} \varepsilon \phi^{2} \dot{\phi} R\left(\eta, \Upsilon, \Upsilon, \Upsilon_{\theta}\right) \\
& \left.+\dot{\phi} \frac{\partial w}{\partial \theta}+\phi \dot{\psi}<\frac{\partial \eta}{\partial x_{0}}, \Upsilon_{\theta}>_{e}+\varepsilon^{2} L(w, \eta)+Q(w, \eta)+O\left(\varepsilon^{3}\right)\right) \\
& -\frac{\varepsilon^{2}}{2} \partial_{s}\left(\phi^{2}+2 \phi w+\frac{1}{3} \varepsilon^{2} \phi^{4} R\left(\Upsilon, \Upsilon_{\theta}, \Upsilon, \Upsilon_{\theta}\right)\right. \\
& \left.+\frac{2}{3} \varepsilon \phi^{3} R\left(\Upsilon, \Upsilon_{\theta}, \eta, \Upsilon_{\theta}\right)+\varepsilon^{2} L(w, \eta)+Q(w, \eta)+O\left(\varepsilon^{3}\right)\right) \\
& =-\varepsilon^{2} \phi \dot{\phi}+O\left(\varepsilon^{4}\right)+\varepsilon^{2} L(w, \eta)+\varepsilon^{2} Q(w, \eta), \\
& <\partial_{\theta}, \nabla_{\partial_{\theta}} \partial_{\theta}>=\frac{1}{2} \partial_{\theta}<\partial_{\theta}, \partial_{\theta}> \\
& =\frac{\varepsilon^{2}}{2} \partial_{\theta}\left(\phi^{2}+2 \phi w+\frac{1}{3} \varepsilon^{2} \phi^{4} R\left(\Upsilon, \Upsilon_{\theta}, \Upsilon, \Upsilon_{\theta}\right)\right. \\
& \left.+\frac{2}{3} \varepsilon \phi^{3} R\left(\Upsilon, \Upsilon_{\theta}, \eta, \Upsilon_{\theta}\right)+\varepsilon^{2} L(w, \eta)+Q(w, \eta)+O\left(\varepsilon^{3}\right)\right) \\
& =O\left(\varepsilon^{4}\right)+\varepsilon^{2} L(w, \eta)+\varepsilon^{2} Q(w, \eta) \text {, } \\
& \nabla_{\partial_{\theta}} \partial_{\theta}=\varepsilon \frac{\partial w}{\partial \theta} \Upsilon_{\theta}+\varepsilon \frac{\partial^{2} w}{\partial \theta^{2}} \Upsilon+\varepsilon^{2}(\phi+w)^{2} \nabla \Upsilon_{\theta} \Upsilon_{\theta} \\
& +\varepsilon^{2}\left(\frac{\partial w}{\partial \theta}\right)^{2} \nabla \Upsilon \Upsilon+\varepsilon^{2}(\phi+w) \frac{\partial w}{\partial \theta}\left(\nabla \Upsilon_{\theta} \Upsilon+\nabla \Upsilon \Upsilon_{\theta}\right) \\
& <\frac{\dot{\phi}}{\phi} X_{0}, \varepsilon \frac{\partial w}{\partial \theta} \Upsilon_{\theta}>=\varepsilon^{3} L(w, \eta)+\varepsilon Q(w, \eta), \\
& <\frac{\dot{\phi}}{\phi} X_{0}, \varepsilon \frac{\partial^{2} w}{\partial \theta^{2}} \Upsilon>=\varepsilon^{4} L(w, \eta)+\varepsilon Q(w, \eta), \\
& <\frac{\dot{\phi}}{\phi} X_{0}, \varepsilon^{2}(\phi+w)^{2} \nabla \Upsilon_{\theta} \Upsilon_{\theta}>=\varepsilon^{2} \frac{\dot{\phi}}{\phi}(\phi+w)^{2}<X_{0}, \nabla \Upsilon_{\theta} \Upsilon_{\theta}>
\end{aligned}
$$

Notice that

$$
<X_{0}, \nabla_{\Upsilon_{\theta}} \Upsilon_{\theta}>=\Upsilon_{\theta}<X_{0}, \Upsilon_{\theta}>-<\Upsilon_{\Upsilon_{\theta}} X_{0}, \Upsilon_{\theta}>
$$


We recall $\Upsilon\left(x_{0}, x_{1}, x_{2}\right)=\frac{\left(x_{1}-\eta_{1}, x_{2}-\eta_{2}\right)}{\sqrt{\left(x_{1}-\eta_{1}\right)^{2}+\left(x_{2}-\eta_{2}\right)^{2}}}=(\cos \theta, \sin \theta)$ and $\Upsilon_{\theta}\left(x_{0}, x_{1}, x_{2}\right)=$ $\frac{\left(-x_{2}+\eta_{2}, x_{1}-\eta_{1}\right)}{\sqrt{\left(x_{1}-\eta_{1}\right)^{2}+\left(x_{2}-\eta_{2}\right)^{2}}}=(-\sin \theta, \cos \theta)$. We denote $\left(-x_{2}+\eta_{2}, x_{1}-\eta_{1}\right)$ by $\tilde{\partial}_{\theta}$, and $\sqrt{\left(x_{1}-\eta_{1}\right)^{2}+\left(x_{2}-\eta_{2}\right)^{2}}$ by $\tilde{r}$

$$
\begin{aligned}
\Upsilon_{\theta}<X_{0}, \Upsilon_{\theta}>= & \frac{1}{\tilde{r}} \tilde{\partial}_{\theta}<X_{0},-\sin \theta X_{1}+\cos \theta X_{2}> \\
= & \frac{1}{\tilde{r}}<X_{0},-\Upsilon>+(-\sin \theta) \Upsilon_{\theta}<X_{0}, X_{1}>+\cos \theta \Upsilon_{\theta}<X_{0}, X_{2}> \\
= & \frac{2}{3} \varepsilon \phi R\left(\Upsilon_{\theta}, X_{0}, \Upsilon, \Upsilon_{\theta}\right)+\frac{2}{3} R\left(\Upsilon_{\theta}, X_{0}, \eta, \Upsilon_{\theta}\right)-\frac{2}{3} R\left(\Upsilon, X_{0}, \eta, \Upsilon\right) \\
& +\varepsilon L(w, \eta)+\varepsilon^{-1} Q(w, \eta)+O\left(\varepsilon^{2}\right),
\end{aligned}
$$

where we calculate $\Upsilon_{\theta}<X_{0}, X_{i}>$ directly from Lemma 2.2. To calculate $<$ $\nabla \Upsilon_{\theta} X_{0}, \Upsilon_{\theta}>$ we can regard $\Upsilon_{\theta}$ as constant in $\left(x_{0}, x_{1}, x_{2}\right)$ coordinate.

$$
\begin{aligned}
<\nabla_{\Upsilon_{\theta}} X_{0}, \Upsilon_{\theta}> & =\frac{1}{2} X_{0}<\Upsilon_{\theta}, \Upsilon_{\theta}> \\
& =O\left(\varepsilon^{2}\right)+\varepsilon L(w, \eta)+Q(w, \eta) .
\end{aligned}
$$

We have

$$
\begin{aligned}
&<\frac{\dot{\phi}}{\phi} X_{0}, \varepsilon^{2}(\phi+w)^{2} \nabla \Upsilon_{\theta} \Upsilon_{\theta}>= \frac{2}{3} \varepsilon^{3} \dot{\phi} \phi^{2} R\left(\Upsilon_{\theta}, X_{0}, \Upsilon, \Upsilon_{\theta}\right) \\
&+\frac{2}{3} \varepsilon^{2} \phi \dot{\phi}\left(R\left(\Upsilon_{\theta}, X_{0}, \eta, \Upsilon_{\theta}\right)-R\left(\Upsilon, X_{0}, \eta, \Upsilon\right)\right) \\
&+\varepsilon^{3} L(w, \eta)+\varepsilon Q(w, \eta)+O\left(\varepsilon^{4}\right) . \\
&<\frac{\dot{\phi}}{\phi} X_{0}, \varepsilon^{2}\left(\frac{\partial w}{\partial \theta}\right)^{2} \nabla \Upsilon \Upsilon=\varepsilon^{3} Q(w, \eta) . \\
&<\frac{\dot{\phi}}{\phi} X_{0}, \varepsilon^{2}(\phi+w) \frac{\partial w}{\partial \theta} \nabla_{\Upsilon_{\theta}} \Upsilon=\varepsilon^{3} L(w, \eta)+\varepsilon^{2} Q(w, \eta) . \\
&<\frac{\dot{\phi}}{\phi} X_{0}, \varepsilon^{2}(\phi+w) \frac{\partial w}{\partial \theta} \nabla_{\Upsilon} \Upsilon_{\theta}>=\varepsilon^{3} L(w, \eta)+\varepsilon^{2} Q(w, \eta) .
\end{aligned}
$$

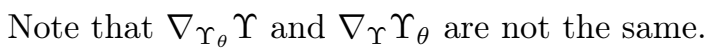

$$
\begin{gathered}
<-\frac{\dot{\psi}}{\phi} \Upsilon, \varepsilon \frac{\partial w}{\partial \theta} \Upsilon_{\theta}>=\varepsilon^{3} L(w, \eta)+\varepsilon Q(w, \eta) . \\
<-\frac{\dot{\psi}}{\phi} \Upsilon, \varepsilon \frac{\partial^{2} w}{\partial \theta^{2}} \Upsilon>=-\varepsilon \frac{\dot{\psi}}{\phi} \frac{\partial^{2} w}{\partial \theta^{2}}+\varepsilon^{3} L(w, \eta)+\varepsilon^{3} Q(w, \eta) .
\end{gathered}
$$




$$
\begin{aligned}
<-\frac{\dot{\psi}}{\phi} \Upsilon, \varepsilon^{2}(\phi+w)^{2} \nabla \Upsilon_{\theta} \Upsilon_{\theta}> & =-\varepsilon^{2} \frac{\dot{\psi}}{\phi}(\phi+w)^{2}<\Upsilon, \nabla \Upsilon_{\theta} \Upsilon_{\theta}> \\
& =-\varepsilon^{2} \frac{\dot{\psi}}{\phi}(\phi+w)^{2}\left(\Upsilon_{\theta}<\Upsilon, \Upsilon_{\theta}>-<\nabla \Upsilon_{\theta} \Upsilon, \Upsilon_{\theta}>\right),
\end{aligned}
$$

First we have

$$
\begin{aligned}
\Upsilon_{\theta}<\Upsilon, \Upsilon_{\theta}>= & \frac{1}{\tilde{r}}\left(<\Upsilon_{\theta}, \Upsilon_{\theta}>-<\Upsilon, \Upsilon>\right) \\
& +\left(-\sin \theta \cos \theta \Upsilon_{\theta}<X_{1}, X_{1}>+\sin \theta \cos \theta \Upsilon_{\theta}<X_{2}, X_{2}>\right. \\
& \left.+\left(\cos ^{2} \theta-\sin ^{2} \theta\right) \Upsilon_{\theta}<X_{1}, X_{2}>\right) \\
= & \frac{1}{3} R\left(\Upsilon, \Upsilon_{\theta}, \eta, \Upsilon_{\theta}\right)+\varepsilon L(w, \eta)+\varepsilon^{-1} Q(w, \eta)+O\left(\varepsilon^{2}\right), \\
<\nabla \Upsilon_{\theta} \Upsilon, \Upsilon_{\theta}>= & \frac{1}{\tilde{r}}<\nabla_{\tilde{r} \Upsilon_{\theta}} \Upsilon, \Upsilon_{\theta}> \\
= & \frac{1}{\tilde{r}}<\nabla \Upsilon \tilde{r} \Upsilon_{\theta}, \Upsilon_{\theta}> \\
= & \frac{1}{\tilde{r}}<\Upsilon_{\theta}, \Upsilon_{\theta}>+<\nabla \Upsilon_{\theta}, \Upsilon_{\theta}>
\end{aligned}
$$

From

$$
\begin{aligned}
\frac{1}{\tilde{r}}<\Upsilon_{\theta}, \Upsilon_{\theta}>= & \frac{1}{\tilde{r}}+\frac{1}{3} \varepsilon \phi R\left(\Upsilon, \Upsilon_{\theta}, \Upsilon, \Upsilon_{\theta}\right)+\frac{2}{3} R\left(\Upsilon, \Upsilon_{\theta}, \eta, \Upsilon_{\theta}\right) \\
& +\varepsilon L(w, \eta)+\varepsilon^{-1} Q(w, \eta)+O\left(\varepsilon^{2}\right) \\
<\nabla \Upsilon \Upsilon_{\theta}, \Upsilon_{\theta}>= & \frac{1}{2} \Upsilon<\Upsilon_{\theta}, \Upsilon_{\theta}> \\
= & \frac{1}{3} \varepsilon \phi R\left(\Upsilon, \Upsilon_{\theta}, \Upsilon, \Upsilon_{\theta}\right)+\frac{1}{3} R\left(\Upsilon, \Upsilon_{\theta}, \eta, \Upsilon_{\theta}\right) \\
& +O\left(\varepsilon^{2}\right)+\varepsilon L(w, \eta)+Q(w, \eta) .
\end{aligned}
$$

We get

$$
\begin{aligned}
& <\nabla_{\Upsilon_{\theta}} \Upsilon, \Upsilon_{\theta}>=\frac{1}{\tilde{r}}+\frac{2}{3} \varepsilon \phi R\left(\Upsilon, \Upsilon_{\theta}, \Upsilon, \Upsilon_{\theta}\right)+R\left(\Upsilon, \Upsilon_{\theta}, \eta, \Upsilon_{\theta}\right) \\
& +\varepsilon L(w, \eta)+\varepsilon^{-1} Q(w, \eta)+O\left(\varepsilon^{2}\right) \\
& <-\frac{\dot{\psi}}{\phi} \Upsilon, \varepsilon^{2}(\phi+w)^{2} \nabla \Upsilon_{\theta} \Upsilon_{\theta}>=\varepsilon\left(\phi^{2}+\tau_{0}\right)+\varepsilon \frac{\dot{\psi}}{\phi} w+\frac{2}{3} \varepsilon^{3} \phi^{2} \dot{\psi} R\left(\Upsilon, \Upsilon_{\theta}, \Upsilon, \Upsilon_{\theta}\right) \\
& +\frac{2}{3} \phi \dot{\psi} R\left(\Upsilon, \Upsilon_{\theta}, \eta, \Upsilon_{\theta}\right) \\
& +\varepsilon^{3} L(w, \eta)+\varepsilon Q(w, \eta)+O\left(\varepsilon^{4}\right) . \\
& <-\frac{\dot{\psi}}{\phi} \Upsilon, \varepsilon^{2}\left(\frac{\partial w}{\partial \theta}\right)^{2} \nabla_{\Upsilon} \Upsilon>=\varepsilon^{3} Q(w, \eta) .
\end{aligned}
$$




$$
\begin{aligned}
& <-\frac{\dot{\psi}}{\phi} \Upsilon, \varepsilon^{2}(\phi+w) \frac{\partial w}{\partial \theta} \nabla_{\Upsilon_{\theta}} \Upsilon>=\varepsilon^{3} L(w, \eta)+\varepsilon^{2} Q(w, \eta) . \\
& <-\frac{\dot{\psi}}{\phi} \Upsilon, \varepsilon^{2}(\phi+w) \frac{\partial w}{\partial \theta} \nabla \Upsilon \Upsilon_{\theta}>=\varepsilon^{3} L(w, \eta)+\varepsilon^{2} Q(w, \eta) .
\end{aligned}
$$

We collect all the terms and get

$$
\begin{aligned}
& <N_{0}, \nabla_{\partial_{\theta}} \partial_{\theta}> \\
= & \varepsilon\left(\phi^{2}+\tau_{0}\right)-\varepsilon \frac{\dot{\psi}}{\phi} \frac{\partial^{2} w}{\partial \theta^{2}}+\varepsilon \frac{\dot{\psi}}{\phi} w \\
& +\frac{2}{3} \varepsilon^{3} \phi^{2} \dot{\psi} R\left(\Upsilon, \Upsilon_{\theta}, \Upsilon, \Upsilon_{\theta}\right)+\frac{2}{3} \varepsilon^{3} \dot{\phi} \phi^{2} R\left(\Upsilon_{\theta}, X_{0}, \Upsilon, \Upsilon_{\theta}\right) \\
& +\frac{2}{3} \varepsilon^{2} \phi \dot{\phi} R\left(\Upsilon_{\theta}, X_{0}, \eta, \Upsilon_{\theta}\right)-\frac{2}{3} \varepsilon^{2} \phi \dot{\phi} R\left(\Upsilon, X_{0}, \eta, \Upsilon\right) \\
& +\frac{2}{3} \phi \dot{\psi} R\left(\Upsilon, \Upsilon_{\theta}, \eta, \Upsilon_{\theta}\right)+\varepsilon^{3} L(w, \eta)+\varepsilon Q(w, \eta)+O\left(\varepsilon^{4}\right) .
\end{aligned}
$$

For

$$
<N, \nabla_{\partial_{\theta}} \partial_{s}>=<N_{0}+a_{1} \partial_{s}+a_{2} \partial_{\theta}, \nabla_{\partial_{\theta}} \partial_{s}>
$$

we don't need very precise expansion because $g^{s \theta}$ is small relatively.

$$
\begin{gathered}
<\partial_{s}, \nabla_{\partial_{\theta}} \partial_{s}>=O\left(\varepsilon^{4}\right)+\varepsilon^{2} L(w, \eta)+\varepsilon^{2} Q(w, \eta), \\
<\partial_{\theta}, \nabla_{\partial_{\theta}} \partial_{s}>=\varepsilon^{2} \phi \dot{\phi}+O\left(\varepsilon^{4}\right)+\varepsilon^{2} L(w, \eta)+\varepsilon^{2} Q(w, \eta) . \\
<N_{0}, \nabla_{\partial_{\theta}} \partial_{s}>=O\left(\varepsilon^{3}\right)+\varepsilon^{2} L(w, \eta)+\varepsilon Q(w, \eta) .
\end{gathered}
$$

Now we can calculate the mean curvature. From (9), (10), (11), (56), (57) and (61) we know

$$
\begin{aligned}
g^{s s}<k N, \nabla_{\partial_{s}} \partial_{s}>= & \varepsilon^{-2} \phi^{-2}\left(1-\left(\varepsilon^{2} \dot{\psi}^{2} R\left(\Upsilon, X_{0}, \Upsilon, X_{0}\right)+2 \varepsilon \phi^{-1} \dot{\psi}^{2} R\left(\Upsilon, X_{0}, \eta, X_{0}\right)\right.\right. \\
& +\frac{4}{3} \varepsilon \phi^{-1} \dot{\phi} \dot{\psi} R\left(\Upsilon, X_{0}, \eta, \Upsilon\right)+2 \phi^{-2} \dot{\phi} \frac{\partial w}{\partial s}+2 \phi^{-2} \dot{\phi} \dot{\psi}<\Upsilon \frac{\partial \eta}{\partial x_{0}}>_{e} \\
& \left.\left.+\varepsilon^{2} L(w, \eta)+Q(w, \eta)+O\left(\varepsilon^{3}\right)\right)\right) \\
& \left(a_{1} \frac{\varepsilon^{2}}{2}\left(2 \phi \dot{\phi}+L(w, \eta)+Q(w, \eta)+O\left(\varepsilon^{2}\right)\right)\right. \\
& +a_{2} \varepsilon^{2}\left(O\left(\varepsilon^{2}\right)+L(w, \eta)+Q(w, \eta)\right) \\
& +\varepsilon\left(\phi^{2}-\tau_{0}\right)-\varepsilon \frac{\dot{\psi}}{\phi} \frac{\partial^{2} w}{\partial s^{2}}-\varepsilon^{2} \frac{\dot{\psi}^{3}}{\phi}<\frac{\partial^{2} \eta}{\partial x_{0}^{2}}, \Upsilon>-\varepsilon \frac{\dot{\psi} \ddot{\psi}}{\phi}<\frac{\partial \eta}{\partial x_{0}}, \Upsilon> \\
& +\varepsilon^{3}\left(\phi \dot{\phi} \ddot{\psi}+2 \dot{\phi}^{2} \dot{\psi}+\dot{\psi}^{3}\right) R\left(\Upsilon, X_{0}, \Upsilon, X_{0}\right) \\
& +\varepsilon^{2}\left(2 \dot{\phi} \ddot{\psi}+2 \frac{\dot{\phi}^{2} \dot{\psi}}{\phi}+\frac{\dot{\psi}^{3}}{\phi}\right) R\left(\Upsilon, X_{0}, \eta, X_{0}\right) \\
& +\varepsilon^{2}\left(\frac{2}{3} \dot{\phi} \ddot{\phi}+\frac{2}{3} \frac{\dot{\phi}^{3}}{\phi}-\frac{2}{3} \dot{\psi} \ddot{\psi}\right) R\left(\Upsilon, X_{0}, \eta, \Upsilon\right) \\
& \left.+\varepsilon^{3} L(w, \eta)+\varepsilon Q(w, \eta)+O\left(\varepsilon^{4}\right)\right)
\end{aligned}
$$




$$
\begin{aligned}
= & \varepsilon^{-2} \phi^{-2}\left(a_{1} \varepsilon^{2} \phi \dot{\phi}+\varepsilon\left(\phi^{2}-\tau_{0}\right)-\varepsilon \frac{\dot{\psi}}{\phi} \frac{\partial^{2} w}{\partial s^{2}}-2 \varepsilon\left(\phi^{2}-\tau_{0}\right) \frac{\dot{\phi}}{\phi^{2}} \frac{\partial w}{\partial s}\right. \\
& -\varepsilon^{2} \frac{\dot{\psi}^{3}}{\phi}<\frac{\partial^{2} \eta}{\partial x_{0}^{2}}, \Upsilon>-\varepsilon\left(\frac{\dot{\psi} \ddot{\psi}}{\phi}+2\left(\phi^{2}-\tau_{0}\right) \frac{\dot{\phi} \dot{\psi}}{\phi^{2}}\right)<\frac{\partial \eta}{\partial x_{0}}, \Upsilon> \\
& +\varepsilon^{3}\left(\phi \dot{\phi} \ddot{\psi}+2 \dot{\phi}^{2} \dot{\psi}+\dot{\psi}^{3}-\left(\phi^{2}-\tau_{0}\right) \dot{\psi}^{2}\right) R\left(\Upsilon, X_{0}, \Upsilon, X_{0}\right) \\
& +\varepsilon^{2}\left(2 \dot{\phi} \ddot{\psi}+2 \frac{\dot{\phi}^{2} \dot{\psi}}{\phi}+\frac{\dot{\psi}^{3}}{\phi}-2 \frac{\dot{\psi}^{2}}{\phi}\left(\phi^{2}-\tau_{0}\right)\right) R\left(\Upsilon, X_{0}, \eta, X_{0}\right) \\
& +\varepsilon^{2}\left(\frac{2}{3} \dot{\phi} \ddot{\phi}+\frac{2}{3} \frac{\dot{\phi}^{3}}{\phi}-\frac{2}{3} \dot{\psi} \ddot{\psi}-\frac{4}{3}\left(\phi^{2}-\tau_{0}\right) \frac{\dot{\phi} \dot{\psi}}{\phi}\right) R\left(\Upsilon, X_{0}, \eta, \Upsilon\right) \\
& \left.+\varepsilon^{3} L(w, \eta)+\varepsilon Q(w, \eta)+O\left(\varepsilon^{4}\right)\right) .
\end{aligned}
$$

From (9), 10), 11), 62), 633 and (64) we know

$$
\begin{aligned}
g^{\theta \theta}<k N, \nabla_{\partial_{\theta}} \partial_{\theta}>= & \varepsilon^{-2} \phi^{-2}\left(1-\left(2 \phi^{-1} w+\frac{1}{3} \varepsilon^{2} \phi^{2} R\left(\Upsilon, \Upsilon_{\theta}, \Upsilon, \Upsilon_{\theta}\right)\right.\right. \\
& \left.\left.+\frac{2}{3} \varepsilon \phi R\left(\Upsilon, \Upsilon_{\theta}, \eta, \Upsilon_{\theta}\right)+\varepsilon^{2} L(w, \eta)+Q(w, \eta)+O\left(\varepsilon^{3}\right)\right)\right) \\
& \left(a_{1}\left(-\varepsilon^{2} \phi \dot{\phi}+O\left(\varepsilon^{4}\right)+\varepsilon^{2} L(w, \eta)+\varepsilon^{2} Q(w, \eta)\right)\right. \\
& +a_{2}\left(O\left(\varepsilon^{4}\right)+\varepsilon^{2} L(w, \eta)+\varepsilon^{2} Q(w, \eta)\right) \\
& +\varepsilon\left(\phi^{2}+\tau_{0}\right)-\varepsilon \frac{\dot{\psi}}{\phi} \frac{\partial^{2} w}{\partial \theta^{2}}+\varepsilon \frac{\dot{\psi}}{\phi} w+\frac{2}{3} \varepsilon^{3} \phi^{2} \dot{\psi} R\left(\Upsilon, \Upsilon_{\theta}, \Upsilon_{,} \Upsilon_{\theta}\right) \\
& +\frac{2}{3} \varepsilon^{3} \dot{\phi} \phi^{2} R\left(\Upsilon_{\theta}, X_{0}, \Upsilon, \Upsilon_{\theta}\right)+\frac{2}{3} \varepsilon^{2} \phi \dot{\phi} R\left(\Upsilon_{\theta}, X_{0}, \eta, \Upsilon_{\theta}\right) \\
& -\frac{2}{3} \varepsilon^{2} \phi \dot{\phi} R\left(\Upsilon, X_{0}, \eta, \Upsilon\right)+\frac{2}{3} \varepsilon^{2} \phi \dot{\psi} R\left(\Upsilon, \Upsilon_{\theta}, \eta, \Upsilon_{\theta}\right) \\
& \left.+\varepsilon^{3} L(w, \eta)+\varepsilon Q(w, \eta)+O\left(\varepsilon^{4}\right)\right) \\
= & \varepsilon^{-2} \phi^{-2}\left(-\varepsilon^{2} a_{1} \phi \dot{\phi}+\varepsilon\left(\phi^{2}+\tau_{0}\right)-\varepsilon \frac{\dot{\psi}}{\phi} \frac{\partial^{2} w}{\partial \theta^{2}}-\varepsilon \frac{\dot{\psi}}{\phi} w\right. \\
& +\frac{1}{3} \varepsilon^{3} \phi^{2} \dot{\psi} R\left(\Upsilon, \Upsilon_{\theta}, \Upsilon, \Upsilon_{\theta}\right)+\frac{2}{3} \varepsilon^{3} \dot{\phi} \phi^{2} R\left(\Upsilon_{\theta}, X_{0}, \Upsilon, \Upsilon_{\theta}\right) \\
& +\frac{2}{3} \varepsilon^{2} \phi \dot{\phi} R\left(\Upsilon_{\theta}, X_{0}, \eta, \Upsilon_{\theta}\right)-\frac{2}{3} \varepsilon^{2} \phi \dot{\phi} R\left(\Upsilon, X_{0}, \eta, \Upsilon\right) \\
& \left.+\varepsilon^{3} L(w, \eta)+\varepsilon Q(w, \eta)+O\left(\varepsilon^{4}\right)\right)
\end{aligned}
$$


From (9), 10), (11), 65), 66) and 67) we know

$$
\begin{aligned}
g^{s \theta}<k N, \nabla_{\partial_{\theta}} \partial_{s}>= & \varepsilon^{-2} \phi^{-2}\left(-\left(\frac{2}{3} \varepsilon^{2} \phi \dot{\psi} R\left(\Upsilon, X_{0}, \Upsilon, \Upsilon_{\theta}\right)+\frac{2}{3} \varepsilon \dot{\psi}\left(R\left(\Upsilon, X_{0}, \eta, \Upsilon_{\theta}\right)\right.\right.\right. \\
& \left.+R\left(\eta, X_{0}, \Upsilon, \Upsilon_{\theta}\right)\right)+\frac{1}{3} \varepsilon \dot{\phi} R\left(\eta, \Upsilon, \Upsilon, \Upsilon_{\theta}\right) \\
& \left.\left.+\phi^{-2} \dot{\phi} \frac{\partial w}{\partial \theta}+\phi^{-1} \dot{\psi}<\frac{\partial \eta}{\partial x_{0}}, \Upsilon_{\theta}>_{e}+\varepsilon^{2} L(w, \eta)+Q(w, \eta)+O\left(\varepsilon^{3}\right)\right)\right) \\
& \left(a_{1}\left(O\left(\varepsilon^{4}\right)+\varepsilon^{2} L(w, \eta)+\varepsilon^{2} Q(w, \eta)\right)\right. \\
& +a_{2}\left(\varepsilon^{2} \phi \dot{\phi}+O\left(\varepsilon^{4}\right)+\varepsilon^{2} L(w, \eta)+\varepsilon^{2} Q(w, \eta)\right) \\
& \left.+O\left(\varepsilon^{3}\right)+\varepsilon^{2} L(w, \eta)+\varepsilon Q(w, \eta)\right) \\
= & \varepsilon^{-2} \phi^{-2}\left(\varepsilon^{3} L(w, \eta)+\varepsilon Q(w, \eta)+O\left(\varepsilon^{4}\right)\right) .
\end{aligned}
$$

So

$$
\begin{aligned}
H= & \frac{1}{k} \varepsilon^{-2} \phi^{-2}\left(2 \varepsilon \phi^{2}-\varepsilon \frac{\dot{\psi}}{\phi}\left(\frac{\partial^{2} w}{\partial s^{2}}+\frac{\partial^{2} w}{\partial \theta^{2}}\right)-2 \varepsilon\left(\phi^{2}-\tau_{0}\right) \frac{\dot{\phi}}{\phi^{2}} \frac{\partial w}{\partial s}-\varepsilon \frac{\dot{\psi}}{\phi} w\right. \\
& -\varepsilon^{2} \frac{\dot{\psi}^{3}}{\phi}<\frac{\partial^{2} \eta}{\left(\partial x_{0}\right)^{2}}, \Upsilon>-\varepsilon\left(\frac{\dot{\psi} \ddot{\psi}}{\phi}+2\left(\phi^{2}-\tau_{0}\right) \frac{\dot{\phi} \dot{\psi}}{\phi^{2}}\right)<\frac{\partial \eta}{\partial x_{0}}, \Upsilon> \\
& +\varepsilon^{3}\left(\phi \dot{\phi} \ddot{\psi}+2 \dot{\phi}^{2} \dot{\psi}+\dot{\psi}^{3}-\left(\phi^{2}-\tau_{0}\right) \dot{\psi}^{2}\right) R\left(\Upsilon, X_{0}, \Upsilon, X_{0}\right) \\
& +\frac{1}{3} \varepsilon^{3} \phi^{2} \dot{\psi} R\left(\Upsilon, \Upsilon_{\theta}, \Upsilon, \Upsilon_{\theta}\right)+\frac{2}{3} \varepsilon^{3} \dot{\phi} \phi^{2} R\left(\Upsilon_{\theta}, X_{0}, \Upsilon, \Upsilon_{\theta}\right) \\
& +\varepsilon^{2}\left(2 \dot{\phi} \ddot{\psi}+2 \frac{\dot{\phi}^{2} \dot{\psi}}{\phi}+\frac{\dot{\psi}^{3}}{\phi}-2 \frac{\dot{\psi}^{2}}{\phi}\left(\phi^{2}-\tau_{0}\right)\right) R\left(\Upsilon, X_{0}, \eta, X_{0}\right) \\
& +\varepsilon^{2}\left(\frac{2}{3} \dot{\phi} \ddot{\phi}+\frac{2}{3} \frac{\dot{\phi}^{3}}{\phi}-\frac{2}{3} \dot{\psi} \ddot{\psi}-\frac{4}{3}\left(\phi^{2}-\tau_{0}\right) \frac{\dot{\phi} \dot{\psi}}{\phi}-\frac{2}{3} \phi \dot{\phi}\right) R\left(\Upsilon, X_{0}, \eta, \Upsilon\right) \\
& +\frac{2}{3} \varepsilon^{2} \phi \dot{\phi} R\left(\Upsilon_{\theta}, X_{0}, \eta, \Upsilon_{\theta}\right) \\
& \left.+\varepsilon^{3} L(w, \eta)+\varepsilon Q(w, \eta)+O\left(\varepsilon^{4}\right)\right)
\end{aligned}
$$

From 12 we know

$$
\begin{aligned}
\frac{1}{k}= & 1-\frac{\varepsilon^{2}}{2} \dot{\phi}^{2} R\left(\Upsilon, X_{0}, \Upsilon, X_{0}\right)-\varepsilon \frac{\dot{\phi}^{2}}{\phi} R\left(\Upsilon, X_{0}, \eta, X_{0}\right) \\
& +\frac{2}{3} \varepsilon \frac{\dot{\phi} \dot{\psi}}{\phi} R\left(\Upsilon, X_{0}, \eta, \Upsilon\right)+\varepsilon^{2} L(w, \eta)+Q(w, \eta)+O\left(\varepsilon^{3}\right) .
\end{aligned}
$$

So at last we get (13). 


\section{B The proof of Lemma 3.1 and Lemma 3.6}

The proof of Lemma 3.1. The goal is to prove that

$$
\begin{aligned}
& \int_{a_{1}}^{b_{1}} \frac{1}{\phi}\left(2 \dot{\phi} \ddot{\psi}+2 \frac{\dot{\phi}^{2} \dot{\psi}}{\phi}+\frac{\dot{\psi}^{3}}{\phi}-2 \frac{\dot{\psi}^{2}}{\phi}\left(\phi^{2}-\tau_{0}\right)-2 \phi \dot{\phi}^{2}\right) d \psi \cdot \int_{a_{1}}^{b_{1}} \frac{\phi^{2}}{\dot{\psi}^{3}} d \psi \\
= & \left(b_{1}-a_{1}\right)^{2},
\end{aligned}
$$

where $\left[a_{1}, b_{1}\right]$ is one period for $\phi(\psi)$.

Proof.

$$
\int_{a_{1}}^{b_{1}} \frac{\phi^{2}}{\dot{\psi}^{3}} d \psi=\int_{a_{1}}^{b_{1}} \frac{1}{\phi}\left(1+\phi_{\psi}^{2}\right)^{\frac{3}{2}} d \psi
$$

From

$$
\phi_{\psi \psi}-\phi^{-1}\left(1+\phi_{\psi}^{2}\right)+2\left(1+\phi_{\psi}^{2}\right)^{\frac{3}{2}}=0
$$

we have

$$
\int_{a_{1}}^{b_{1}} \frac{\phi^{2}}{\dot{\psi}^{3}} d \psi=\int_{a_{1}}^{b_{1}}\left(\frac{1+\phi_{\psi}^{2}}{2 \phi^{2}}-\frac{\phi_{\psi \psi}}{2 \phi}\right) d \psi .
$$

Note that

$$
\begin{aligned}
\int_{a_{1}}^{b_{1}}-\frac{\phi_{\psi \psi}}{2 \phi} d \psi & =-\frac{1}{2}\left(\left.\frac{\phi_{\psi}}{2 \phi}\right|_{a_{1}} ^{b_{1}}-\int_{a_{1}}^{b_{1}} \phi_{\psi} \frac{-\phi_{\psi}}{\phi^{2}} d \psi\right) \\
& =-\frac{1}{2} \int_{a_{1}}^{b_{1}} \phi_{\psi} \frac{-\phi_{\psi}}{\phi^{2}} d \psi
\end{aligned}
$$

So we have

$$
\int_{a_{1}}^{b_{1}} \frac{\phi^{2}}{\dot{\psi}^{3}} d \psi=\int_{a_{1}}^{b_{1}} \frac{1}{2 \phi^{2}} d \psi .
$$

Also from direct computation one can get

$$
\begin{aligned}
& \int_{a_{1}}^{b_{1}} \frac{1}{\phi}\left(2 \dot{\phi} \ddot{\psi}+2 \frac{\dot{\phi}^{2} \dot{\psi}}{\phi}+\frac{\dot{\psi}^{3}}{\phi}-2 \frac{\dot{\psi}^{2}}{\phi}\left(\phi^{2}-\tau_{0}\right)-2 \phi \dot{\phi}^{2}\right) d \psi \\
= & \int_{a_{1}}^{b_{1}}\left(\phi^{2}\left(2 \phi_{\psi \psi}\left(1+\phi_{\psi}^{2}\right)^{-\frac{5}{2}}-\left(1+\phi_{\psi}^{2}\right)^{-\frac{5}{2}} \phi_{\psi \psi} \phi_{\psi}^{2}\right)\right. \\
& \left.+\phi\left(3\left(1+\phi_{\psi}^{2}\right)^{-\frac{3}{2}} \phi_{\psi}^{2}+\left(1+\phi_{\psi}^{2}\right)^{-\frac{3}{2}}\right)\right) d \psi \\
= & \int_{a_{1}}^{b_{1}}\left(2 \phi^{2}+\frac{2 \phi^{2} \phi_{\psi \psi}}{\left(1+\phi_{\psi}^{2}\right)^{\frac{3}{2}}}\right) d \psi .
\end{aligned}
$$

We assume that

$$
\phi(s)=\sqrt{\tau_{0}} \exp (\sigma(s)) .
$$


Then we have

$$
\begin{aligned}
\dot{\psi} & =\sqrt{\tau_{0}} \exp (\sigma(s)) \sqrt{1-\sigma_{s}^{2}}, \\
\phi_{\psi} & =\frac{\sigma_{s}}{\sqrt{1-\sigma_{s}^{2}}}, \\
\phi_{\psi \psi} & =\frac{\sigma_{s s}}{\sqrt{\tau_{0}} \exp (\sigma)\left(1-\sigma_{s}^{2}\right)^{2}} .
\end{aligned}
$$

Also we have

$$
\begin{aligned}
1-\sigma_{s}^{2} & =4 \tau_{0} \cosh ^{2} \sigma, \\
\sigma_{s s} & =-2 \tau_{0} \sinh 2 \sigma .
\end{aligned}
$$

For $\phi$ there are two particular points in one period such that $\sigma=0$. Suppose these two points are

$$
s=s_{1}, s=s_{2}
$$

and suppose $\left[s_{1}, s_{3}\right]$ is one period for $\phi(s)$. Note that $s_{2} \in\left(s_{1}, s_{3}\right)$.

Here we use one particular property of Delaunay surface

$$
\sigma\left(s_{2}-t\right)=-\sigma\left(s_{2}+t\right) .
$$

Then from direct calculation we know

$$
\begin{aligned}
& \int_{a_{1}}^{b_{1}} \frac{1}{2 \phi^{2}} d \phi=\int_{s_{1}}^{s_{3}} \cosh ^{2} \sigma d s \\
& \int_{a_{1}}^{b_{1}}\left(2 \phi^{2}+\frac{2 \phi^{2} \phi_{\psi \psi}}{\left(1+\phi_{\psi}^{2}\right)^{\frac{3}{2}}}\right) d \psi=4 \tau_{0}^{2} \int_{s_{1}}^{s_{3}} \cosh ^{2} \sigma d s
\end{aligned}
$$

and

$$
b_{1}-a_{1}=2 \tau_{0} \int_{s_{1}}^{s_{3}} \cosh ^{2} \sigma d s
$$

So we know

$$
\begin{aligned}
& \int_{a_{1}}^{b_{1}} \frac{1}{\phi}\left(2 \dot{\phi} \ddot{\psi}+2 \frac{\dot{\phi}^{2} \dot{\psi}}{\phi}+\frac{\dot{\psi}^{3}}{\phi}-2 \frac{\dot{\psi}^{2}}{\phi}\left(\phi^{2}-\tau_{0}\right)-2 \phi \dot{\phi}^{2}\right) d \psi \cdot \int_{a_{1}}^{b_{1}} \frac{\phi^{2}}{\dot{\psi}^{3}} d \psi \\
= & \left(b_{1}-a_{1}\right)^{2} .
\end{aligned}
$$

Proof of Lemma 3.6. Without loss of generality, we may assume $a_{\tau_{0}}=$ $a_{1}, b_{\tau_{0}}=b_{1}$. The goal is to prove

$$
\int_{a_{1}}^{b_{1}} \phi\left(\frac{\partial \phi}{\partial \psi}\right) \phi^{-2}\left(\frac{2}{3} \dot{\phi} \ddot{\phi}+\frac{2}{3} \frac{\dot{\phi}^{3}}{\phi}-\frac{2}{3} \dot{\psi} \ddot{\psi}-\frac{4}{3}\left(\phi^{2}-\tau_{0}\right) \frac{\dot{\phi} \dot{\psi}}{\phi}+\frac{4}{3} \phi \dot{\phi} \dot{\psi}\right) d \psi=0
$$

which is equivalent to

$$
\int_{a_{1}}^{b_{1}} \phi_{\psi}^{2} \phi^{-2} \dot{\psi}\left(\phi \ddot{\phi}+\dot{\phi}^{2}-2\left(\phi^{2}+\tau_{0}\right)\left(\phi^{2}-\tau_{0}\right)\right) d \psi=0 .
$$


Proof. Note that

$$
\begin{aligned}
& \int_{a_{1}}^{b_{1}} \phi_{\psi}^{2} \phi^{-2} \dot{\psi}\left(\phi \ddot{\phi}+\dot{\phi}^{2}-2\left(\phi^{2}+\tau_{0}\right)\left(\phi^{2}-\tau_{0}\right)\right) d \psi=0 \\
& =\int_{a_{1}}^{b_{1}} \frac{\sigma_{s}^{2}}{1-\sigma_{s}^{2}} \frac{1}{\tau \exp (2 \sigma)} \tau_{0} \exp (2 \sigma)\left(1-\sigma_{s}^{2}\right)\left(\phi \ddot{\phi}+\dot{\phi}^{2}-2\left(\phi^{2}+\tau_{0}\right)\left(\phi^{2}-\tau_{0}\right)\right) d \psi \\
& =\int_{a_{1}}^{b_{1}} \sigma_{s}^{2}\left(\phi \ddot{\phi}+\dot{\phi}^{2}-2\left(\phi^{2}+\tau_{0}\right)\left(\phi^{2}-\tau_{0}\right)\right) d \psi \text {. } \\
& \int_{a_{1}}^{b_{1}} \sigma_{s}^{2}\left(\phi \ddot{\phi}+\dot{\phi}^{2}\right) d \psi \\
& =\int_{a_{1}}^{b_{1}} \sigma_{s}^{2}(\phi \dot{\phi})_{s} d s \\
& =-\int_{a_{1}}^{b_{1}} \phi \dot{\phi} 2 \sigma_{s} \sigma_{s s} d s \\
& =-2 \int_{a_{1}}^{b_{1}} \exp (\sigma) \exp (\sigma) \sigma_{s}^{2} \sigma_{s s} d s \\
& =4 \tau_{0}^{2} \int_{a_{1}}^{b_{1}} \exp (2 \sigma)\left(1-4 \tau_{0} \cosh ^{2}(\sigma)\right) \sinh (2 \sigma) d s \\
& =2 \tau_{0}^{2} \int_{a_{1}}^{b_{1}}\left(1-4 \tau_{0} \cosh ^{2}(\sigma)\right) \sinh ^{2}(2 \sigma) d s . \\
& -2 \int_{a_{1}}^{b_{1}} \sigma_{s}^{2}\left(\phi^{2}+\tau_{0}\right)\left(\phi^{2}-\tau_{0}\right) d s \\
& =-2 \int_{a_{1}}^{b_{1}} \sigma_{s}^{2}\left(\tau_{0} \exp (2 \sigma)+\tau_{0}\right)\left(\tau_{0} \exp (2 \sigma)-\tau_{0}\right) d s \\
& =-2 \tau_{0}^{2} \int_{a_{1}}^{b_{1}} \sigma_{s}^{2} 4 \sinh (\sigma) \cosh (\sigma) \exp (2 \sigma) d s \\
& =-2 \tau_{0}^{2} \int_{a_{1}}^{b_{1}} \sigma_{s}^{2} 2 \sinh (2 \sigma) \exp (2 \sigma) d s \\
& =-2 \tau_{0}^{2} \int_{a_{1}}^{b_{1}} \sigma_{s}^{2} \sinh ^{2}(2 \sigma) d s \\
& =-2 \tau_{0}^{2} \int_{a_{1}}^{b_{1}}\left(1-4 \tau_{0} \cosh ^{2}(\sigma)\right) \sinh ^{2}(2 \sigma) d s .
\end{aligned}
$$

So we proved that

$$
\int_{a_{1}}^{b_{1}} \phi_{\psi}^{2} \phi^{-2} \dot{\psi}\left(\phi \ddot{\phi}+\dot{\phi}^{2}-2\left(\phi^{2}+\tau_{0}\right)\left(\phi^{2}-\tau_{0}\right)\right) d \psi=0 .
$$




\section{The proof of Lemma 3.11}

First we prove

$$
\left|\Phi^{\prime}(\psi)-1\right| \leq C\left(\tau_{0}\right)\left(\varepsilon+\|\xi\|_{C^{0}}+\varepsilon^{2}\|\mu\|_{C^{0}}+\varepsilon^{2}|\omega|\right) .
$$

Note that

$$
\Phi^{\prime}(\psi)=\frac{d \Phi(\psi)}{d s_{0}} \frac{d s_{0}}{d l} \frac{d l}{d \psi}
$$

where

$$
d l=\sqrt{<\partial_{s_{0}}, \partial_{s_{0}}>d s_{0}^{2}+<\partial_{\tau}, \partial_{\tau}>d \tau^{2}}
$$

is the arc length of $(\phi(\psi), \zeta(\psi))$. On the curve $(\phi(\psi), \zeta(\psi))$ we have

$$
1=\sqrt{<\partial_{s_{0}}, \partial_{s_{0}}>\left(\frac{d s_{0}}{d l}\right)^{2}+<\partial_{\tau}, \partial_{\tau}>\left(\frac{d \tau}{d \psi}\right)^{2}\left(\frac{d \psi}{d l}\right)^{2}}
$$

In $C\left(\tau_{0}\right)\left(\varepsilon+\|\xi\|_{C^{0}}+\varepsilon^{2}\|\mu\|_{C^{0}}+\varepsilon^{2}|\omega|\right)$ neighborhood of $\left(\phi_{\tau(0)}(\psi), \zeta_{\tau(0)}(\psi)\right)$,

$$
\left|<\partial_{s_{0}}, \partial_{s_{0}}>-1\right| \leq C\left(\tau_{0}\right)\left(\varepsilon+\|\xi\|_{C^{0}}+\varepsilon^{2}\|\mu\|_{C^{0}}+\varepsilon^{2}|\omega|\right)
$$

And

$$
\left|\frac{d \tau}{d \psi}\right| \leq C \varepsilon\left(\varepsilon+\|\xi\|_{C^{0}}+\varepsilon^{2}\|\mu\|_{C^{0}}+\varepsilon^{2}|\omega|\right) .
$$

Note that

$$
\begin{aligned}
\frac{d l}{d \psi} & =\sqrt{\left(\frac{d \phi}{d \psi}\right)^{2}+\left(\frac{d \zeta}{d \psi}\right)^{2}} \\
& =\sqrt{\zeta^{2}+\left(\phi^{-1}\left(1+\phi^{2}\right)-(2+\rho)\left(1+\zeta^{2}\right)^{\frac{3}{2}}\right)^{2}}
\end{aligned}
$$

and

$$
\begin{aligned}
\frac{d s_{0}}{d \Phi(\psi)} & =\sqrt{\left(\frac{d \phi_{\tau(0)}}{d \Phi(\psi)}\right)^{2}+\left(\frac{d \zeta_{\tau(0)}}{d \Phi(\psi)}\right)^{2}} \\
& =\left.\sqrt{\zeta_{\tau(0)}^{2}+\left(\phi_{\tau(0)}^{-1}\left(1+\phi_{\tau(0)}^{2}\right)-2\left(1+\zeta_{\tau(0)}^{2}\right)^{\frac{3}{2}}\right)^{2}}\right|_{\Phi(\psi)}
\end{aligned}
$$

So we have

$$
\left|\frac{d l}{d \psi} / \frac{d s_{0}}{d \Phi(\psi)}-1\right| \leq C\left(\tau_{0}\right)\left(\varepsilon+\|\xi\|_{C^{0}}+\varepsilon^{2}\|\mu\|_{C^{0}}+\varepsilon^{2}|\omega|\right)
$$

Note that $\frac{d s_{0}}{d \Phi(\psi)}$ has both positive upper bound and positive lower bound which only depend on $\tau_{0}$ and so does $\frac{d l}{d \psi}$ when $\varepsilon$ is sufficiently small.

So we know $\left(\frac{d \psi}{d l}\right)^{2}$ is bounded and together with $68,69,70$ we get

$$
\left|\frac{d s_{0}}{d l}-1\right| \leq C\left(\tau_{0}\right)\left(\varepsilon+\|\xi\|_{C^{0}}+\varepsilon^{2}\|\mu\|_{C^{0}}+\varepsilon^{2}|\omega|\right) .
$$


From 71) (72), we get

$$
\begin{aligned}
& \left|\Phi^{\prime}(\psi)-1\right|=\left|\frac{\left.\sqrt{\zeta^{2}+\left(\phi^{-1}\left(1+\zeta^{2}\right)-(2+\rho)\left(1+\zeta^{2}\right)^{\frac{3}{2}}\right)^{2}}\right|_{\psi}}{\left.\sqrt{\zeta_{\tau(0)}^{2}+\left(\phi_{\tau(0)}^{-1}\left(1+\phi_{\tau(0)}^{2}\right)-2\left(1+\zeta_{\tau(0)}^{2}\right)^{\frac{3}{2}}\right)^{2}}\right|_{\Phi(\psi)}} \frac{d s_{0}}{d l}-1\right| \\
& \leq C\left(\tau_{0}\right)\left(\varepsilon+\|\xi\|_{C^{0}}+\varepsilon^{2}\|\mu\|_{C^{0}}+\varepsilon^{2}|\omega|\right) .
\end{aligned}
$$

By integration we know that

$$
|\Phi(\psi)-\psi| \leq \frac{C\left(\tau_{0}\right)}{\varepsilon}\left(\varepsilon+\|\xi\|_{C^{0}}+\varepsilon^{2}\|\mu\|_{C^{0}}+\varepsilon^{2}|\omega|\right) .
$$

\section{The proof of Lemma 3.15}

$$
\begin{aligned}
& \left(\begin{array}{cc}
\beta_{1}\left(\psi_{i}\right) & \beta_{2}\left(\psi_{i}\right) \\
\frac{\partial \beta_{1}}{\partial \psi}\left(\psi_{i}\right) & \frac{\partial \beta_{2}}{\partial \psi}\left(\psi_{i}\right)
\end{array}\right) \\
= & \left(\begin{array}{cc}
1+e_{11}^{i} & e_{12}^{i} \\
\kappa+e_{21}^{i} & 1+e_{22}^{i}
\end{array}\right) \cdots\left(\begin{array}{cc}
1+e_{11}^{1} & e_{12}^{1} \\
\kappa+e_{21}^{1} & 1+e_{22}^{1}
\end{array}\right) \\
= & \left(\begin{array}{cc}
A_{11}^{i} & A_{12}^{i} \\
A_{21}^{i} & A_{22}^{i}
\end{array}\right)
\end{aligned}
$$

where $\left|e_{k l}^{j}\right| \leq C\left(\tau_{0}\right)\left(\varepsilon^{2}+\|\xi\|_{C^{0}}+\varepsilon^{2}\|\mu\|_{C^{0}}+\varepsilon^{2}|\omega|\right)$.

Lemma D.1. For $\varepsilon$ sufficiently small, if $\left|a_{i j}\right| \leq C\left(\tau_{0}\right)\left(\varepsilon^{2}+\|\xi\|_{C^{0}}+\varepsilon^{2}\|\mu\|_{C^{0}}+\right.$ $\left.\varepsilon^{2}|\omega|\right)$ and for $k \in \mathbb{Z}, k \leq \frac{C\left(\tau_{0}\right)}{\varepsilon},|f| \leq k C\left(\tau_{0}\right)\left(\varepsilon^{2}+\|\xi\|_{C^{0}}+\varepsilon^{2}\|\mu\|_{C^{0}}+\varepsilon^{2}|\omega|\right)$, there are $\tilde{a}_{11}, \tilde{a}_{21}, \tilde{a}_{22}, \tilde{f}$ such that the following holds

$$
\left(\begin{array}{cc}
1+a_{11} & a_{12} \\
\kappa+a_{21} & 1+a_{22}
\end{array}\right)\left(\begin{array}{cc}
1 & f \\
0 & 1
\end{array}\right)=\left(\begin{array}{cc}
1 & \tilde{f} \\
0 & 1
\end{array}\right)\left(\begin{array}{cc}
1+\tilde{a}_{11} & 0 \\
\kappa+\tilde{a}_{21} & 1+\tilde{a}_{22}
\end{array}\right),
$$

with

$$
\begin{aligned}
|\tilde{f}| & \leq(k+2) C\left(\tau_{0}\right)\left(\varepsilon^{2}+\|\xi\|_{C^{0}}+\varepsilon^{2}\|\mu\|_{C^{0}}+\varepsilon^{2}|\omega|\right), \\
\left|\tilde{a}_{11}\right|,\left|\tilde{a}_{22}\right| & \leq(\kappa k+1) C\left(\tau_{0}\right)\left(\varepsilon^{2}+\|\xi\|_{C^{0}}+\varepsilon^{2}\|\mu\|_{C^{0}}+\varepsilon^{2}|\omega|\right) .
\end{aligned}
$$

Proof. (of Lemma D.1) Note that

$$
\begin{aligned}
\left|\left(\kappa+a_{21}\right) f\right| & \leq \frac{C\left(\tau_{0}\right)}{\varepsilon}\left(\varepsilon^{2}+\|\xi\|_{C^{0}}+\varepsilon^{2}\|\mu\|_{C^{0}}+\varepsilon^{2}|\omega|\right) \\
& \leq C\left(\tau_{0}\right)\left(1+C_{1}+C_{2}+C_{3}\right) \varepsilon .
\end{aligned}
$$

So we can choose $\varepsilon$ sufficiently small such that

$$
1+a_{22}+f\left(\kappa+a_{21}\right) \geq 1-C\left(\tau_{0}, C_{1}, C_{2}, C_{3}\right) \varepsilon
$$


and it is invertible. Then one can check directly that

$$
\left\{\begin{array}{l}
\tilde{a}_{11}=a_{11}-\left(\kappa+a_{21}\right) \frac{\left(1+a_{11}\right) f+a_{12}}{1+a_{22}+f\left(\psi_{1}+a_{21}\right)}, \\
\tilde{a}_{21}=a_{21} \\
\tilde{a}_{22}=\left(\kappa+a_{21}\right) f+a_{22} \\
\tilde{f}=\frac{\left(1+a_{11}\right) f+a_{12}}{1+a_{22}+f\left(\kappa+a_{21}\right)}
\end{array}\right.
$$

satisfies 73 . So one can easily get

$$
\begin{aligned}
|\tilde{f}| & \leq(k+2) C\left(\tau_{0}\right)\left(\varepsilon^{2}+\|\xi\|_{C^{0}}+\varepsilon^{2}\|\mu\|_{C^{0}}+\varepsilon^{2}|\omega|\right), \\
\left|\tilde{a}_{11}\right|,\left|\tilde{a}_{22}\right| & \leq(\kappa k+1) C\left(\tau_{0}\right)\left(\varepsilon^{2}+\|\xi\|_{C^{0}}+\varepsilon^{2}\|\mu\|_{C^{0}}+\varepsilon^{2}|\omega|\right) .
\end{aligned}
$$

Note that

$$
\begin{aligned}
& \left(\begin{array}{cc}
1+e_{11}^{i} & e_{12}^{i} \\
\kappa+e_{21}^{i} & 1+e_{22}^{i}
\end{array}\right) \cdots\left(\begin{array}{cc}
1+e_{11}^{1} & e_{12}^{1} \\
\kappa+e_{21}^{1} & 1+e_{22}^{1}
\end{array}\right) \\
= & \left(\begin{array}{cc}
1+e_{11}^{i} & e_{12}^{i} \\
\kappa+e_{21}^{i} & 1+e_{22}^{i}
\end{array}\right) \cdots\left(\begin{array}{cc}
1+e_{11}^{2} & e_{12}^{2} \\
\kappa+e_{21}^{2} & 1+e_{22}^{2}
\end{array}\right) \\
& \left(\begin{array}{cc}
1 & -f_{12}^{1} \\
0 & 1
\end{array}\right)\left(\begin{array}{cc}
1+\tilde{e}_{11}^{1} & 0 \\
\kappa+\tilde{e}_{21}^{1} & 1+\tilde{e}_{22}^{1}
\end{array}\right) .
\end{aligned}
$$

Because $i$ is at most as big as $\frac{L_{\Gamma}}{\varepsilon \psi_{1}}$ and $\left|f_{12}^{1}\right|,\left|e_{i j}^{k}\right| \leq C\left(\tau_{0}\right)\left(\varepsilon^{2}+\|\xi\|_{C^{0}}+\varepsilon^{2}\|\mu\|_{C^{0}}+\right.$ $\left.\varepsilon^{2}|\omega|\right)$, we can use Lemma D.1 for $i$ times. Note that by induction

$$
\left|f_{12}^{j}\right| \leq 2 j C\left(\tau_{0}\right)\left(\varepsilon^{2}+\|\xi\|_{C^{0}}+\varepsilon^{2}\|\mu\|_{C^{0}}+\varepsilon^{2}|\omega|\right)
$$

and $2 j \leq \frac{2 L_{\Gamma}}{\varepsilon \psi_{1}} \leq \frac{C\left(\tau_{0}\right)}{\varepsilon}$. At last we get

$$
\begin{aligned}
& \left(\begin{array}{cc}
A_{11}^{i} & A_{12}^{i} \\
A_{21}^{i} & A_{22}^{i}
\end{array}\right) \\
= & \left(\begin{array}{cc}
1 & -f_{12}^{i} \\
0 & 1
\end{array}\right)\left(\begin{array}{cc}
1+\tilde{e}_{11}^{i} & 0 \\
\kappa+\tilde{e}_{21}^{i} & 1+\tilde{e}_{22}^{i}
\end{array}\right) \cdots\left(\begin{array}{cc}
1+\tilde{e}_{11}^{1} & 0 \\
\kappa+\tilde{e}_{21}^{1} & 1+\tilde{e}_{22}^{1}
\end{array}\right)
\end{aligned}
$$

where

$$
\left|f_{12}^{i}\right|,\left|\tilde{e}_{k l}^{j}\right| \leq \frac{C\left(\tau_{0}\right)}{\varepsilon}\left(\varepsilon^{2}+\|\xi\|_{C^{0}}+\varepsilon^{2}\|\mu\|_{C^{0}}+\varepsilon^{2}|\omega|\right) .
$$

If we replace $-f_{12}^{i}$ and $\tilde{e}_{k l}^{j}$ by $E r=\varepsilon C\left(\tau_{0}\right)\left(1+C_{1}+C_{2}+C_{3}\right)$ then all $A_{k l}^{i}$ will become bigger. Note that

$$
\begin{aligned}
& \left(\begin{array}{cc}
1+E r & 0 \\
\kappa+E r & 1+E r
\end{array}\right) \cdots\left(\begin{array}{cc}
1+E r & 0 \\
\kappa+E r & 1+E r
\end{array}\right) \\
= & \left(\begin{array}{cc}
(1+E r)^{i} & 0 \\
i(\kappa+E r)(1+E r)^{i-1} & (1+E r)^{i}
\end{array}\right)
\end{aligned}
$$


Note that $i \leq \frac{C\left(\tau_{0}\right)}{\varepsilon}$. So

$$
\begin{aligned}
(1+E r)^{i} & \leq \exp C\left(\tau_{0}\right)\left(1+C_{1}+C_{2}+C_{3}\right) \\
i(\kappa+E r)(1+E r)^{i-1} & \leq \frac{1}{\varepsilon} \exp C\left(\tau_{0}\right)\left(1+C_{1}+C_{2}+C_{3}\right)
\end{aligned}
$$

In the same way if we replace $-f_{12}^{i}$ all $\tilde{e}_{k l}^{j}$ by $-E r$ then all $A_{k l}^{i}$ will become smaller. Note that

$$
\begin{aligned}
& \left(\begin{array}{cc}
1-E r & 0 \\
\kappa-E r & 1-E r
\end{array}\right) \cdots\left(\begin{array}{cc}
1-E r & 0 \\
\kappa-E r & 1-E r
\end{array}\right) \\
= & \left(\begin{array}{cc}
(1-E r)^{i} & 0 \\
i(\kappa-E r)(1-E r)^{i-1} & (1-E r)^{i}
\end{array}\right)
\end{aligned}
$$

and

$$
\begin{aligned}
(1-E r)^{i} & \geq \exp \left(-C\left(\tau_{0}\right)\left(1+C_{1}+C_{2}+C_{3}\right)\right) \\
i(\kappa-E r)(1-E r)^{i-1} & \geq \frac{1}{\varepsilon} \exp \left(-C\left(\tau_{0}\right)\left(1+C_{1}+C_{2}+C_{3}\right)\right)
\end{aligned}
$$

So we know

$$
\begin{array}{rc}
\exp \left(-C\left(\tau_{0}\right)\left(1+C_{1}+C_{2}+C_{3}\right)\right) & \leq A_{22}^{i} \leq \exp C\left(\tau_{0}\right)\left(1+C_{1}+C_{2}+C_{3}\right), \\
\exp \left(-C\left(\tau_{0}\right)\left(1+C_{1}+C_{2}+C_{3}\right)\right) \varepsilon^{-1} & \leq A_{21}^{i} \leq \exp C\left(\tau_{0}\right)\left(1+C_{1}+C_{2}+C_{3}\right) \varepsilon^{-1}, \\
\left|A_{12}^{i}\right| & \leq C\left(\tau_{0}, C_{1}, C_{2}, C_{3}\right) \varepsilon \exp C\left(\tau_{0}\right)\left(1+C_{1}+C_{2}+C_{3}\right) .
\end{array}
$$

There is another way to calculate

$$
\begin{aligned}
& \left(\begin{array}{ll}
A_{11}^{i} & A_{12}^{i} \\
A_{21}^{i} & A_{22}^{i}
\end{array}\right) \\
& =\left(\begin{array}{cc}
1+e_{11}^{i} & e_{12}^{i} \\
\kappa+e_{21}^{i} & 1+e_{22}^{i}
\end{array}\right) \cdots\left(\begin{array}{cc}
1+e_{11}^{1} & e_{12}^{1} \\
\kappa+e_{21}^{1} & 1+e_{22}^{1}
\end{array}\right) \\
& =\left(\begin{array}{cc}
1+\bar{e}_{11}^{i} & \bar{e}_{12}^{i} \\
\kappa+\bar{e}_{21}^{i} & 1+\bar{e}_{22}^{i}
\end{array}\right)\left(\begin{array}{cc}
1 & h_{12}^{1} \\
0 & 1
\end{array}\right) \cdots\left(\begin{array}{cc}
1+e_{11}^{1} & e_{12}^{1} \\
\kappa+e_{21}^{1} & 1+e_{22}^{1}
\end{array}\right) \\
& =\cdots \\
& =\left(\begin{array}{cc}
1+\bar{e}_{11}^{i} & \bar{e}_{12}^{i} \\
\kappa+\bar{e}_{21}^{i} & 1+\bar{e}_{22}^{i}
\end{array}\right) \cdots\left(\begin{array}{cc}
1+\bar{e}_{11}^{1} & \bar{e}_{12}^{1} \\
\kappa+\bar{e}_{21}^{1} & 1+\bar{e}_{22}^{1}
\end{array}\right)\left(\begin{array}{cc}
1 & h_{12}^{i} \\
0 & 1
\end{array}\right) .
\end{aligned}
$$

And we can prove that

$$
\exp \left(-\left(C+C_{1}+C_{2}+C_{3}\right)\right) \leq A_{11}^{i} \leq \exp \left(C+C_{1}+C_{2}+C_{3}\right) .
$$

\section{E The proof of Lemma 3.17}

1. $\beta_{\mu}$ estimate From

$$
\left.\frac{d}{d t} \phi_{\xi, \mu+t \Delta \mu, \omega, \tau(0)}(\psi)\right|_{t=0}=\beta_{\mu}(\psi)
$$


we have

$$
\left\{\begin{array}{l}
\mathcal{L}_{\xi, \mu, \omega, \tau(0)} \beta_{\mu}(\psi)=-\varepsilon^{3}\left(1+\phi_{\psi}^{2}\right)^{\frac{3}{2}} \Delta \mu \\
\beta_{\mu}(0)=0 \\
\beta_{\mu}^{\prime}(0)=0
\end{array}\right.
$$

We have

$$
\begin{aligned}
& \beta_{\mu}(\psi)=-\varepsilon^{3} \int_{0}^{\psi} R(t)^{-1}\left(\beta_{2}(\psi) \beta_{1}(t)-\beta_{1}(\psi) \beta_{2}(t)\right)\left(1+\phi_{\psi}^{2}(t)\right)^{\frac{3}{2}} \Delta \mu(t) d t, \\
& \beta_{\mu}^{\prime}(\psi)=-\varepsilon^{3} \int_{0}^{\psi} R(t)^{-1}\left(\beta_{2}^{\prime}(\psi) \beta_{1}(t)-\beta_{1}^{\prime}(\psi) \beta_{2}(t)\right)\left(1+\phi_{\psi}^{2}(t)\right)^{\frac{3}{2}} \Delta \mu(t) d t .
\end{aligned}
$$

So from 43

$$
\left\|\beta_{\mu}(\psi)\right\|_{C_{\varepsilon}^{1}} \leq C\left(\tau_{0}, C_{1}, C_{2}, C_{3}\right) \varepsilon\|\Delta \mu\|_{C^{0}} .
$$

2. $\beta_{\xi}$ estimate From

$$
\begin{gathered}
\left.\frac{d}{d t} \phi_{\xi+t \Delta \xi, \mu, \omega, \tau(0)}(\psi)\right|_{t=0}=\beta_{\xi}(\psi) \\
\left\{\begin{array}{l}
\mathcal{L}_{\xi, \mu, \omega, \tau(0)} \beta_{\xi}(\psi)=-\varepsilon F_{4}\left(\phi, \phi_{\psi}\right)\left(1+\phi_{\psi}^{2}\right)^{\frac{3}{2}} \Delta \xi \\
\beta_{\xi}(0)=0, \\
\beta_{\xi}^{\prime}(0)=0 .
\end{array}\right.
\end{gathered}
$$

It is easy to prove that

$$
\left\|\beta_{\xi}(\psi)\right\|_{C_{\varepsilon}^{1}} \leq \frac{C\left(\tau_{0}, C_{1}, C_{2}, C_{3}\right)}{\varepsilon}\|\Delta \xi\|_{C^{0}} .
$$

We are going to prove 47.

Consider

$$
\begin{aligned}
& \left|\left(\beta_{\xi}, \beta_{\xi}^{\prime}\right) \cdot\left(\tau_{\phi}, \tau_{\zeta}\right)\right|_{\bar{\psi}} \mid \\
= & \left|\frac{d}{d t} \tau\right|_{\bar{\psi}} \mid \\
= & \left|\frac{d}{d t} \int_{0}^{\bar{\psi}} \phi \phi_{\psi} \rho d \psi\right| \\
= & \mid \int_{0}^{\bar{\psi}} \varepsilon^{2}\left(\frac{\partial \hat{F}_{1}}{\partial \phi} \beta_{\xi}+\frac{\partial \hat{F}_{1}}{\partial \zeta} \beta_{\xi}^{\prime}\right)+\varepsilon^{3}\left(\phi_{\psi} \beta_{\xi}+\phi \beta_{\xi}^{\prime}\right) \mu \\
& +\varepsilon\left(\frac{\partial \hat{F}_{2}}{\partial \phi} \beta_{\xi}+\frac{\partial \hat{F}_{2}}{\partial \zeta} \beta_{\xi}^{\prime}\right) \xi+\varepsilon \hat{F}_{2} \Delta \xi+\varepsilon^{3} \omega\left(\frac{\partial \hat{F}_{3}}{\partial \phi} \beta_{\xi}+\frac{\partial \hat{F}_{3}}{\partial \zeta} \beta_{\xi}^{\prime}\right) d \psi \mid \\
\leq & C\left(\tau_{0}, C_{1}, C_{2}, C_{3}\right)\|\Delta \xi\|_{C^{0}},
\end{aligned}
$$


where

$$
\begin{aligned}
\hat{F}_{1} & =\phi \frac{\partial \phi}{\partial \psi} F_{1}\left(\phi, \phi_{\psi}\right) \star R_{1}, \\
\hat{F}_{2} & =\phi \frac{\partial \phi}{\partial \psi} F_{4}\left(\phi, \phi_{\psi}\right), \\
\hat{F}_{3} & =\phi_{\psi}^{2} .
\end{aligned}
$$

On the points $\psi_{i}$ where $\phi$ attains its local minimum, we have $\tau_{\zeta}=0$ and $\left|\tau_{\phi}\right|$ has uniform positive lower bound which only depends on $\tau_{0}$. So

$$
\begin{aligned}
& \left|\beta_{\xi}\left(\psi_{i}\right)\right| \leq C\left(\tau_{0}, C_{1}, C_{2}, C_{3}\right)\|\Delta \xi\|_{C^{0}} \\
& \left|\beta_{\xi}^{\prime}\left(\psi_{i}\right)\right| \leq \frac{C\left(\tau_{0}, C_{1}, C_{2}, C_{3}\right)}{\varepsilon}\|\Delta \xi\|_{C^{0}} .
\end{aligned}
$$

So from (44), when $\psi \in\left[\psi_{i}, \psi_{i+1}\right]$,

$$
\begin{aligned}
& \| \beta_{\xi}(\psi)-\left[\beta_{\xi}\left(\psi_{i}\right) h(\tau(0))\left(\left(\psi-\psi_{i}\right) \frac{\partial \phi}{\partial \psi}+v_{i}(\psi)\right)\right. \\
& \left.+\beta_{\xi}^{\prime}\left(\psi_{i}\right) h(\tau(0)) \frac{\partial \phi}{\partial \psi}\right] \|_{C_{\varepsilon}^{1}} \\
\leq & C\left(\tau_{0}, C_{1}, C_{2}, C_{3}\right) \varepsilon\|\Delta \xi\|_{C^{0}} .
\end{aligned}
$$

This tells us that the dominant part of $\beta_{\xi}(\psi)$ is $\beta_{\xi}^{\prime}\left(\psi_{i}\right) h(\tau(0)) \frac{\partial \phi}{\partial \psi}$.

Then we have, for $\bar{\psi} \in\left[\psi_{k}, \psi_{k+1}\right)$

$$
\begin{aligned}
& \left|\int_{0}^{\bar{\psi}} \varepsilon^{2}\left(\frac{\partial \hat{F}_{1}}{\partial \phi} \beta_{\xi}+\frac{\partial \hat{F}_{1}}{\partial \zeta} \beta_{\xi}^{\prime}\right) d \psi\right| \\
\leq & \mid \sum_{i=0}^{k} \int_{\psi_{i-1}}^{\psi_{i}} \varepsilon^{2}\left(\frac{\partial \hat{F}_{1}}{\partial \phi} \beta_{\xi}+\frac{\partial \hat{F}_{1}}{\partial \zeta} \beta_{\xi}^{\prime}\right) d \psi \\
& +\int_{\psi_{k}}^{\bar{\psi}} \varepsilon^{2}\left(\frac{\partial \hat{F}_{1}}{\partial \phi} \beta_{\xi}+\frac{\partial \hat{F}_{1}}{\partial \zeta} \beta_{\xi}^{\prime}\right) d \psi \mid \\
\leq & \frac{C\left(\tau_{0}, C_{1}, C_{2}, C_{3}\right)}{\varepsilon}\|\Delta \xi\|_{C^{0}}\left|\sum_{i=0}^{k} \int_{\psi_{i-1}}^{\psi_{i}} \varepsilon^{2}\left(\frac{\partial \hat{F}_{1}}{\partial \phi} \frac{\partial \phi}{\partial \psi}+\frac{\partial \hat{F}_{1}}{\partial \zeta} \frac{\partial^{2} \phi}{\partial \psi^{2}}\right) d \psi\right| \\
& +C\left(\tau_{0}, C_{1}, C_{2}, C_{3}\right) \varepsilon\|\Delta \xi\|_{C^{0}}^{k} \\
\leq & C\left(\tau_{0}, C_{1}, C_{2}, C_{3}\right) \varepsilon\|\Delta \xi\|_{C^{0}}\left|\sum_{i=0}^{k}\left(\hat{F}_{1}\left(\psi_{i+1}\right)-\hat{F}_{1}\left(\psi_{i}\right)\right)\right| \\
& +C\left(\tau_{0}, C_{1}, C_{2}, C_{3}\right) \varepsilon\|\Delta \xi\|_{C^{0}} \\
\leq & C\left(\tau_{0}, C_{1}, C_{2}, C_{3}\right) \varepsilon\|\Delta \xi\|_{C^{0}} .
\end{aligned}
$$


It is easy to see

$$
\begin{aligned}
& \quad \mid \int_{0}^{\bar{\psi}}\left(\varepsilon^{3}\left(\beta_{\xi} \phi_{\psi}+\phi \beta_{\xi}^{\prime}\right) \mu+\varepsilon\left(\frac{\partial \hat{F}_{2}}{\partial \phi} \beta_{\xi}+\frac{\partial \hat{F}_{2}}{\partial \zeta} \beta_{\xi}^{\prime}\right) \xi\right. \\
& \left.\quad+\varepsilon^{3} \omega\left(\frac{\partial \hat{F}_{3}}{\partial \phi} \beta_{\xi}+\frac{\partial \hat{F}_{3}}{\partial \zeta} \beta_{\xi}^{\prime}\right)\right) d \psi \mid \\
& \leq C\left(\tau_{0}, C_{1}, C_{2}, C_{3}\right) \varepsilon\|\Delta \xi\|_{C^{0}} .
\end{aligned}
$$

The last term is

$$
\int_{0}^{\bar{\psi}} \varepsilon \hat{F}_{2} \Delta \xi d \psi=\varepsilon \int_{0}^{\bar{\psi}} \phi\left(\frac{\partial \phi}{\partial \psi}\right) F_{4}\left(\phi, \phi_{\psi}\right) \Delta \xi d \psi
$$

From Lemma 3.6. using the argument of the proof of Lemma 3.4 also using Corollary 3.13 , we can prove

$$
\left|\int_{0}^{\bar{\psi}} \varepsilon \hat{F}_{2} \Delta \xi d \psi\right| \leq C\left(\tau_{0}, C_{1}, C_{2}, C_{3}\right) \varepsilon\|\Delta \xi\|_{C_{x_{0}}^{1}} .
$$

So we have

$$
\left|\beta_{\xi}\left(\psi_{i}\right)\right| \leq C\left(\tau_{0}, C_{1}, C_{2}, C_{3}\right) \varepsilon\|\Delta \xi\|_{C_{x_{0}}^{1}}
$$

Once we prove that

$$
\left|\beta_{\xi}^{\prime}\left(\psi_{i}\right)\right| \leq C\left(\tau_{0}, C_{1}, C_{2}, C_{3}\right)\|\Delta \xi\|_{C_{x_{0}}^{1}}
$$

we can deduce 477 from 76 .

Note that

$$
\begin{aligned}
& \left|\beta_{\xi}^{\prime}\left(\psi_{i+1}\right)-\left(\beta_{\xi}\left(\psi_{i}\right) \beta_{1, i}^{\prime}\left(\psi_{i+1}\right)+\beta_{\xi}^{\prime}\left(\psi_{i}\right) \beta_{2, i}^{\prime}\left(\psi_{i+1}\right)\right)\right| \\
= & \left|\int_{\psi_{i}}^{\psi_{i+1}} R_{i}^{-1}\left(\beta_{2, i}^{\prime}(\psi) \beta_{1, i}(t)-\beta_{1, i}^{\prime}(\psi) \beta_{2, i}(t)\right) \mathcal{L}_{\xi, \mu, \omega, \tau(0)} \beta_{\xi}(t) d t\right| \\
\leq & C\left(\tau_{0}, C_{1}, C_{2}, C_{3}\right) \varepsilon\|\Delta \xi\|_{C^{0}}
\end{aligned}
$$

where

$$
R_{i}(\psi)=\left|\begin{array}{cc}
\beta_{1, i}(\psi) & \beta_{2, i}(\psi) \\
\beta_{1, i}^{\prime}(\psi) & \beta_{2, i}^{\prime}(\psi)
\end{array}\right|
$$

is the corresponding Wronskian. From Lemma 3.14 and 45 we can deduce

$$
\left|\beta_{\xi}^{\prime}\left(\psi_{i+1}\right)-\beta_{\xi}^{\prime}\left(\psi_{i}\right)\right| \leq C\left(\tau_{0}, C_{1}, C_{2}, C_{3}\right) \varepsilon\left(\|\Delta \xi\|_{C_{x_{0}}^{1}}+\varepsilon \beta_{\xi}^{\prime}\left(\psi_{i}\right)\right) .
$$

From $\beta_{\xi}^{\prime}(0)=0$, by an induction argument, we get

$$
\left|\beta_{\xi}^{\prime}\left(\psi_{i}\right)\right| \leq C\left(\tau_{0}, C_{1}, C_{2}, C_{3}\right)\|\Delta \xi\|_{C_{x_{0}}^{1}} .
$$

So we have

$$
\left\|\beta_{\xi}(\psi)\right\|_{C_{\varepsilon}^{1}} \leq C\left(\tau_{0}, C_{1}, C_{2}, C_{3}\right)\|\Delta \xi\|_{C_{x_{0}}^{1}}
$$


3. $\beta_{\omega}$ estimate From

$$
\left.\frac{d}{d t} \phi_{\xi, \mu, \omega+t, \tau(0)}(\psi)\right|_{t=0}=\beta_{\omega}(\psi),
$$

in the same way as we do for $\beta_{\mu}$ we can get (48).

\section{Proof of 49}

$$
\begin{aligned}
& \frac{d}{d t} \tau(\bar{\psi}) \\
= & \frac{d}{d t} \int_{0}^{\bar{\psi}} \phi \phi_{\psi} \rho d \psi \\
= & \int_{0}^{\bar{\psi}} \varepsilon^{2}\left(\frac{\partial \hat{F}_{1}}{\partial \phi} \beta_{\omega}+\frac{\partial \hat{F}_{1}}{\partial \phi_{\psi}} \beta_{\omega}^{\prime}\right)+\varepsilon^{3}\left(\beta_{\omega} \phi_{\psi}+\phi \beta_{\omega}^{\prime}\right) \mu \\
& +\varepsilon\left(\frac{\partial \hat{F}_{2}}{\partial \phi} \beta_{\omega}+\frac{\partial \hat{F}_{2}}{\partial \phi_{\psi}} \beta_{\omega}^{\prime}\right) R(\xi)+\varepsilon^{3} \omega\left(\frac{\partial \hat{F}_{3}}{\partial \phi} \beta_{\omega}+\frac{\partial \hat{F}_{3}}{\partial \phi_{\psi}} \beta_{\omega}^{\prime}\right)+\varepsilon^{3} \phi_{\psi}^{2} d \psi
\end{aligned}
$$

In the similar way as we did for $\xi$, we can get

$$
\begin{aligned}
& \mid \int_{0}^{\bar{\psi}} \varepsilon^{2}\left(\frac{\partial \hat{F}_{1}}{\partial \phi} \beta_{\omega}+\frac{\partial \hat{F}_{1}}{\partial \phi_{\psi}} \beta_{\omega}^{\prime}\right)+\varepsilon^{3}\left(\beta_{\omega} \phi_{\psi}+\phi \beta_{\omega}^{\prime}\right) \mu \\
& +\varepsilon\left(\frac{\partial \hat{F}_{2}}{\partial \phi} \beta_{\omega}+\frac{\partial \hat{F}_{2}}{\partial \phi_{\psi}} \beta_{\omega}^{\prime}\right) R(\xi)+\varepsilon^{3} \omega\left(\frac{\partial \hat{F}_{3}}{\partial \phi} \beta_{\omega}+\frac{\partial \hat{F}_{3}}{\partial \phi_{\psi}} \beta_{\omega}^{\prime}\right) \mid \\
\leq & C\left(\tau_{0}, C_{1}, C_{2}, C_{3}\right) \varepsilon^{3}
\end{aligned}
$$

The dominant term turns out to be

$$
\int_{0}^{\bar{\psi}} \varepsilon^{3} \phi_{\psi}^{2} d \psi
$$

So for $(\xi, \mu, \omega, \phi(0))$ satisfying 287 , we can choose $\varepsilon$ sufficiently small, such that there is a uniform constant $C_{5}=C_{5}\left(\tau_{0}\right)>0$, which does not depend on $C_{1}, C_{2}, C_{3}, \varepsilon$ such that

$$
\frac{\partial}{\partial \omega} \tau\left(\frac{L_{\Gamma}}{\varepsilon}\right) \geq C_{5}\left(\tau_{0}\right) \varepsilon^{2} .
$$

If we perturb $\phi(0)$ from $\frac{1-\sqrt{1-4 \tau(0)}}{2}$ to $\frac{1-\sqrt{1-4 \tau(0)}}{2}+t$, the linearized function is just $\beta_{1}(\psi)$.

$$
\begin{aligned}
& \quad\left|\frac{\partial}{\partial \phi(0)}\left(\tau\left(\frac{L_{\Gamma}}{\varepsilon}\right)-\tau(0)\right)\right| \\
& =\mid \int_{0}^{\frac{L_{\Gamma}}{\varepsilon}} \varepsilon^{2}\left(\frac{\partial \hat{F}_{1}}{\partial \phi} \beta_{1}+\frac{\partial \hat{F}_{1}}{\partial \zeta} \beta_{1}^{\prime}\right)+\varepsilon^{3}\left(\phi_{\psi} \beta_{1}+\phi \beta_{1}^{\prime}\right) \mu \\
& \quad+\varepsilon\left(\frac{\partial \hat{F}_{2}}{\partial \phi} \beta_{1}+\frac{\partial \hat{F}_{2}}{\partial \zeta} \beta_{1}^{\prime}\right) R(\xi)+\varepsilon^{3} \omega\left(\frac{\partial \hat{F}_{3}}{\partial \phi} \beta_{1}+\frac{\partial \hat{F}_{3}}{\partial \zeta} \beta_{1}^{\prime}\right) d \psi \mid \\
& \quad \leq K_{1}\left(\tau_{0}, C_{1}, C_{2}, C_{3}\right) \varepsilon,
\end{aligned}
$$


where we deal with $\varepsilon^{2}\left(\frac{\partial \hat{F}_{1}}{\partial \phi} \beta_{1}+\frac{\partial \hat{F}_{1}}{\partial \phi_{\psi}} \beta_{1}^{\prime}\right)$ in the same way as we did for $\beta_{\xi}$ (note that we have Lemma 3.15, 3.16.

The estimates for $\left|\frac{\partial \zeta\left(\frac{L_{\Gamma}}{\varepsilon}\right)}{\partial \omega}\right|, \frac{\partial \zeta\left(\frac{L_{\Gamma}}{\varepsilon}\right)}{\partial \phi(0)}$ can be proved from Lemma 3.15 . 3.16 and (48). At last we proved 49p.

\section{Acknowledgement}

The author shows his great respect and thanks to Professor Frank Pacard who gave him this problem as well as many deep insights, including how to deal with the high mode, 1st mode and how to do fixed point argument. The author owes him a lot. The author thanks Professor Gang Tian for long time help, Professor Jie Qing for discussions on the ODE and Jinxing Xu for discussions on Appendix D. The author was supported by FMJH and partially supported by NSFC grant NO.11301284, NO.11571185, NO.11871283.

\section{References}

[1] R. Bettiol and P. Piccione. Delaunay type hypersurfaces in cohomogeneity one manifolds. arXiv:1306.6043, 2013.

[2] C. Delaunay. Sur la surface de révolution dont la courbure moyenne est constante. J. Math. Pure. Appl., 6:309-320, 1841.

[3] J. Eells. The surfaces of delaunay. Math. Intell., pages 53-57, 1987.

[4] L.H. Huang. Foliations by stable spheres with constant mean curvature for isolated systems with general asymptotics. Comm. Math. Phys., 300(2):331373,2010 .

[5] G. Huisken and S.T. Yau. Definition of center of mass for isolated physical systems and unique foliations by stable spheres with constant mean curvature. Invent. Math., 124:281-311, 1996.

[6] N. Korevaar, R. Kusner, and B.Solomon. The structure of complete embedded surfaces with constant mean curvature. J. Differ. Geom., 30(2):465-503, 1989 .

[7] F. Mahmoudi, R. Mazzeo, and F. Pacard. Constant mean curvature hypersurfaces condensing along a submanifold. Geom. Funct. Anal., 16(4):924-958, 2006.

[8] R. Mazzeo and F. Pacard. Constant mean curvature surfaces with delaunay ends. Commun. Anal. Geom., 9(1):169-237, 2001.

[9] R. Mazzeo and F. Pacard. Foliations by constant mean curvature tubes. Commun. Anal. Geom., 13(4):633-670, 2005. 
[10] R. Mazzeo and F. Pacard. Constant curvature foliations in asymptotically hyperbolic spaces. Rev. Mat. Iberoam., 27(1):303-333, 2011.

[11] C. Nerz. Foliation by spheres with constant expansion for isolated systems without asymptotic symmetry. arXiv:1501.02197v1, 2015.

[12] A. Neves and G. Tian. Existence and uniqueness of constant mean curvature foliation of asymptotically hyperbolic 3 -manifolds. Geom. Funct. Anal., 19(3):910-942, 122009.

[13] A. Neves and G. Tian. Existence and uniqueness of constant mean curvature foliation of asymptotically hyperbolic 3-manifolds ii. J. Reine. Angew. Math., 641:69-93, 2010.

[14] F. Pacard and X. Xu. Constant mean curvature spheres in riemannian manifolds. Manuscripta Mathematica., 128(3):175-295, 2009.

[15] R. Rigger. The foliation of asymptotically hyperbolic manifolds by surfaces of constant mean curvature (including the evolution equations and estimates). Manuscripta Math., 113(4):403-421, 2004.

[16] R. Schoen. Uniqueness, symmetry and embeddedness of minimal surfaces. J. Differ. Geom., 18:791-809, 1983.

[17] R. Ye. Foliation by constant mean curvature spheres. Pac. J. Math., 147(2):381-396, 1991. 